\title{
Cancer rehabilitation : evaluation of two group-based programs combining physical and psychosocial training
}

Citation for published version (APA):

Korstjens, I. M. (2008). Cancer rehabilitation : evaluation of two group-based programs combining physical and psychosocial training. [Doctoral Thesis, Maastricht University]. Datawyse / Universitaire Pers Maastricht. https://doi.org/10.26481/dis.20081003ik

Document status and date:

Published: 01/01/2008

DOI:

10.26481/dis.20081003ik

Document Version:

Publisher's PDF, also known as Version of record

Please check the document version of this publication:

- A submitted manuscript is the version of the article upon submission and before peer-review. There can be important differences between the submitted version and the official published version of record.

People interested in the research are advised to contact the author for the final version of the publication, or visit the DOI to the publisher's website.

- The final author version and the galley proof are versions of the publication after peer review.

- The final published version features the final layout of the paper including the volume, issue and page numbers.

Link to publication

\footnotetext{
General rights rights.

- You may freely distribute the URL identifying the publication in the public portal. please follow below link for the End User Agreement:

www.umlib.nl/taverne-license

Take down policy

If you believe that this document breaches copyright please contact us at:

repository@maastrichtuniversity.nl

providing details and we will investigate your claim.
}

Copyright and moral rights for the publications made accessible in the public portal are retained by the authors and/or other copyright owners and it is a condition of accessing publications that users recognise and abide by the legal requirements associated with these

- Users may download and print one copy of any publication from the public portal for the purpose of private study or research.

- You may not further distribute the material or use it for any profit-making activity or commercial gain

If the publication is distributed under the terms of Article $25 \mathrm{fa}$ of the Dutch Copyright Act, indicated by the "Taverne" license above, 


\section{Cancer Rehabilitation}

Evaluation of two group-based programs

combining physical and psychosocial training 
Printed by Datawyse / Universitaire Pers Maastricht Cover design by Fieke Stoffers, ARSFICTIO

Copyright Irene Korstjens, Maastricht, 2008 ISBN: 9789052787374 


\section{Cancer Rehabilitation}

\section{Evaluation of two group-based programs combining physical and psychosocial training}

\section{PROEFSCHRIFT}

ter verkrijging van de graad van doctor aan de Universiteit Maastricht, op gezag van de Rector Magnificus, Prof. mr. G.P.M.F. Mols volgens het besluit van het College van Decanen, in het openbaar te verdedigen op vrijdag 3 oktober 2008 om 14.00 uur

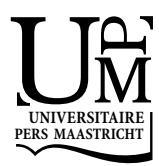




\title{
Promotor
}

Prof.dr. H.W. van den Borne

\section{Copromotor}

\author{
Dr. I. Mesters
}

\section{Beoordelingscommissie}

\author{
Prof.dr. N.K. de Vries (voorzitter) \\ Prof.dr. H. Hillen \\ Prof.dr. M. von Meyenfeldt \\ Prof.dr. J.F.A. Pruyn (Universiteit van Tilburg) \\ Prof. R.M. Ryckman (University of Maine, United States of America)
}

The studies presented in this dissertation were conducted at the School for Public Health and Primary Care (Caphri) acknowledged in 1995 by the Royal Dutch Academy of Science (KNAW).

The "Recovery \& Stability" study project consisted of researchers from the Department of Health Education and Promotion of the Faculty of Health, Medicine and Life Sciences at Maastricht University and the Comprehensive Cancer Center Limburg. This project was funded by the Maastricht University and the Comprehensive Cancer Center Limburg. The "OncoRev" study project consisted of researchers from Maastricht University, the University Medical Center Utrecht, the Erasmus Medical Center Rotterdam, the Comprehensive Cancer Center North-Netherlands, University Medical Center Groningen, and Hanze University Groningen. This project was financed by Maastricht University, the Dutch Cancer Society (UU-2000-2585) and by the Comprehensive Cancer Center North-Netherlands.

Financial support for the publication of this dissertation was provided by Maastricht University, Novartis Pharma and the Comprehensive Cancer Center Limburg. 


\section{Content}

$\begin{array}{lll}\text { Chapter } 1 & \text { General introduction } & 7\end{array}$

Chapter 2 Quality of life of cancer survivors after physical and 21 psychosocial rehabilitation

Chapter 3 Cancer patients' view on rehabilitation and quality of life: 35 a program audit

Chapter 4 Quality of life after self-management cancer rehabilitation: 51 a randomized controlled trial comparing physical and cognitive-behavioral training versus physical training

Chapter 5 Long-term effects on cancer survivors' quality of life of physical training versus physical training combined with cognitive-behavioral therapy: results from a randomized trial

Chapter 6 Effects of cancer rehabilitation on problem-solving and 89 distress: a RCT comparing physical and cognitive-behavioral training versus physical training

Chapter 7 General discussion

References

Summary \& Samenvatting

Dankwoord 


\section{Nothing twice}

Nothing can ever happen twice.

In consequence, the sorry fact is that we arrive here improvised and leave without the chance to practice.

Even if there is no one dumber, If you're the planet's biggest dunce, you can't repeat the class in summer: this course is only offered once.

No day copies yesterday, no two nights will teach what bliss is in precisely the same way, with exactly the same kisses.

One day, perhaps, some idle tongue mentions your name by accident: I feel as if a rose were flung into the room, all hue and scent.

The next day, though you're here with me, I can't help looking at the clock:

A rose? A rose? What could that be?

Is it a flower or a rock?

Why do we treat the fleeting day with so much needless fear and sorrow?

It's in its nature not to stay:

Today is always gone tomorrow.

With smiles and kisses, we prefer to seek accord beneath our star, although we're different (we concur) just as two drops of water are.

Wislawa Szymborska. 
Chapter 1

General introduction 
Because cancer incidence as well as survival rates of persons with cancer are growing (Parkin et al 2001), more and more persons have to manage their cancer as a chronic disease. About $26 \%$ of cancer survivors report decreased quality of life and express a need for professional support in managing physical, psychological and social problems (Van Harten et al 1998).This dissertation addresses the development and evaluation of rehabilitation programs for cancer survivors to improve their quality of life. The first part of this dissertation describes the foundation and evaluation of "Recovery \& Stability" ("Herstel \& Balans") an already longer existing Dutch cancer rehabilitation program. The second part of this dissertation presents studies on the development and evaluation by the "OncoRev" project group (page 4) of "OncoRev", a recently developed cancer rehabilitation program. The present chapter starts with describing medical aspects of cancer, incidence, prevalence and survival rates, and quality of life and rehabilitation needs of cancer survivors. Then, this chapter introduces, subsequently, single-focused physical or psychosocial rehabilitation, multi-modal rehabilitation, the theoretical concepts that were applied in "Recovery \& Stability", and the theoretical foundation for "OncoRev". Lastly, this chapter provides an overview of the dissertation.

\section{Medical aspects of cancer}

Cancer, also called malignant tumors and neoplasms, is a generic term for a group of more than hundred diseases that can affect any part of the body. The incidence of cancer rises dramatically with age, most likely due to risk accumulation over the life course combined with the tendency for cellular repair mechanisms to be less effective as a person grows older. Treatment of cancer is aimed at curing, at prolonging life, and at recovering cancer patients' quality of life. Some of the most common cancer types, such as breast cancer, cervical cancer, and colorectal cancer, have a high cure rate when detected early and treated according to best evidence. The principal methods of treatment are surgery, radiotherapy, and chemotherapy, and these treatments are often combined (www.who.int). 


\section{Incidence, prevalence, and survival rates}

The "Netherlands Cancer Registry", the Dutch nation-wide population-based cancer registry, indicates that, in the year 2003, 73,000 new cancer cases were registered (www.ikc.net.nl). In approximately $10 \%$ of these cases some type of cancer had been diagnosed before; the latest diagnosis might be a recurrence, a metastasis or a different type of cancer. Thus, 66,000 new cancer patients were found. Incidence of breast cancer is the highest $(16 \%$ of all new cancer diagnoses), followed by colorectal cancer (14\%), lung cancer $(12 \%)$ and prostate cancer (11\%). Cancer is, after cardiovascular diseases, the second cause of death in the Netherlands, with 38,200 deaths in 2003. Cancer incidence rises with $1.5 \%$ to $2 \%$ yearly, mainly due to population growth and population aging. In 2015, in the Netherlands, the incidence of cancer is estimated at 96,500 cases and prevalence at 692,500. In addition, survival rates of cancer have also risen, mainly due to early detection, more effective treatment and decreases in incidence of cancer types with low survival rates. Survival rates vary with cancer type and the stage in which cancer is diagnosed. Generally, today's five-year survival rates are about $50 \%$. Due to increases in incidence as well as in survival rates, cancer prevalence rises with $2 \%$ to $3 \%$ yearly. About 400,000 persons were living with cancer in the Netherlands in 2003 , approximately $2.5 \%$ of the population.

\section{Cancer survivors' quality of life and rehabilitation needs}

Many cancer survivors are facing a variety of distressing problems as a result of their disease and its treatment. Approximately 26\% of cancer survivors report decreased quality of life and express a need for professional support in managing their problems following cancer diagnosis and its treatment (Van Harten et al. 1998). Quality of life is a multidimensional construct, including physical, psychological and social dimensions (Ware and Sherbourne 1992; Aaronson et al. 1993). Physical problems (e.g. decreased physical capacity, fatigue), psychological problems (e.g., anxiety, depression, stress, insecurity, grief, decreased self-esteem) and social problems (e.g. hindered job reintegration, social isolation) lead to diminished quality of life (De Haes 1998; Curt et al. 2000; Ganz et al. 1996; Gotay et al. 2002; Tomich and Helgeson 2002). 
It was estimated, that 4,890 Dutch cancer survivors needed rehabilitation in the year 2000 and that 6,900 Dutch cancer survivors may need rehabilitation in the year 2015 (Gijsen et al 2005). These were conservative estimates. Firstly, these estimates were based on the number of new cancer patients yearly with an age between 20 and 69 years: 37,613 in the year 2000 and 53,082 in the year 2015 according the "Netherlands Cancer Registry" (Gijsen et al. 2005). Secondly, these estimates were based on a five-year survival rate of $52 \%$ as rehabilitation is aimed at people with a relatively good prognosis and, lastly, on a rehabilitation need of $25 \%$ of cancer survivors instead of the 26\% reported before (Gijsen et al. 2005; Van Harten et al 1998). In reality, even more Dutch cancer survivors may need rehabilitation as people with an age of minimally 18 years with a life expectancy of minimally one year may enroll in rehabilitation within months after they have completed their medical treatment.

\section{Single focused physical or psychosocial rehabilitation}

Meta-analyses of randomized controlled trials of physical interventions (McNeely et al. 2006; Schmitz et al. 2005) or psychosocial interventions (Meyer and Mark 1995; Rehse and Pukrop 2003) in cancer populations report positive effects on quality of life with small to large effect sizes (ES from 0.28 to 0.84 ). Moreover, a meta-analysis of randomized controlled studies in cancer populations of specific psychosocial interventions that were based on a cognitive-behavioral approach reports large positive effects (ES 1.45) on quality of life (Osborn et al. 2006). Although rehabilitation programs initially were either physical or psychosocial, currently it seems that cancer rehabilitation has started to incorporate physical as well as psychosocial elements. A review of psychological interventions for cancer patients (Andersen 2002) found that the focus has expanded from primarily reducing stress and enhancing quality of life to biobehavioral change (e.g. health behaviors, biological responses, disease outcomes). A review of physical activity after a cancer diagnosis explicitly focused on the psychosocial outcomes of exercise as well (Schwartz 2004). Noticing this trend in looking more broadly at mechanisms and effects in cancer rehabilitation and acknowledging the value of Engel's biopsychosocial model (Engel 1977; Engel 1980; Engel 1997), it might be expected that combining physical and psychosocial interventions in multi-modal cancer rehabilitation enhances improvements in quality of life beyond the benefits of a single focused 
intervention. Therefore, the studies in this dissertation focus on the benefits of multi-modal cancer rehabilitation.

\section{Multi-modal physical and psychosocial rehabilitation}

The first two studies in this dissertation focus on "Recovery \& Stability", the founding Dutch cancer rehabilitation program, combining a physical and a psychosocial module. The program was implemented by the "Comprehensive Cancer Center Limburg" in 1996 in two rehabilitation centers. This 12week rehabilitation program was group-based and consisted of physical training sessions (twice weekly), guided by physiotherapists, and 7 psychosocial sessions, guided by different professionals addressing different themes. To date, cancer rehabilitation based on this multi-modal program is being offered in more than 60 centers throughout the Netherlands and Belgium and is becoming part of regular care. Based on the studies concerning "Recovery \& Stability", the "OncoRev" project group (page 4) developed "OncoRev".

The "Recovery \& Stability" studies demonstrated that combined rehabilitation was feasible and showed promising beneficial effects on cancer survivors' quality of life. At the same time, these studies suggested that there was room for improvement of the program. We also considered that a more rigorous evaluation of the separate modules and a comparison with no intervention was needed. We hypothesized that integrating cognitivebehavioral elements into physical training might enhance the effects of physical training on quality of life and that combining such a comprehensive physical training with a cognitive-behavioral psychosocial training could lead to even greater improvements in cancer survivors' quality of life. These hypotheses were based on the large effect-sizes of cognitive-behavioral interventions in cancer populations (Osborn et al. 2006). Therefore, "OncoRev" was designed as a 12-week group-based multi-modal cancer rehabilitation program combining an innovative comprehensive physical training and a cognitive-behavioral psychosocial training. Both training modules were based on principles of self-management (Creer 2000; Mesters et al. 2002). "OncoRev" started in 2004 in 4 centers in the Netherlands. 


\section{Theoretical concepts in "Recovery \& Stability"}

The rehabilitation program "Recovery \& Stability" was aimed at improving cancer survivors' quality of life. As quality of life includes physical, psychological, and social dimensions (Aaronson et al. 1993; Ware and Sherbourne 1992), the program combined physical and psychosocial modules, and promoted social interaction between the participants. The program was based on the biopsychosocial model (Engel 1977; Engel 1980; Engel 1997). Both the physical module and the psychosocial module were group-based to enhance contacts between fellow survivors and provided opportunities for social comparison (Bogart and Helgeson 2000; Festinger 1954; Stanton et al. 1999), social support (Helgeson and Cohen 1996) and modeling (Bandura 1986). Next, these concepts will be further elaborated.

\section{Biopsychosocial model}

In the biopsychosocial model the individual is part of a hierarchy of natural systems, and can be seen as a system and as a subsystem at the same time (Engel 1977; Engel 1980; Engel 1997). Each (sub) system as a whole has its own characteristics, dynamics and relations. In the continuity of natural systems, every unit is at the same time both a whole and a part. The single individual represents the highest level of the organic hierarchy and at the same time the lowest unit of the social hierarchy. Changes in subsystems influence other subsystems within natural systems, in which cybernetic regulation mechanisms with positive (change) and negative (preservation) feedback loops are working to maintain homeostasis. The biopsychosocial model acknowledges that physical problems may induce psychosocial problems and vice versa. The model further implies, firstly, that physical interventions might affect physical problems as well as psychosocial problems, secondly, that psychosocial interventions have the potential to affect psychosocial and physical problems, and, lastly, that combining such interventions may enhance the effect of the entire intervention. 


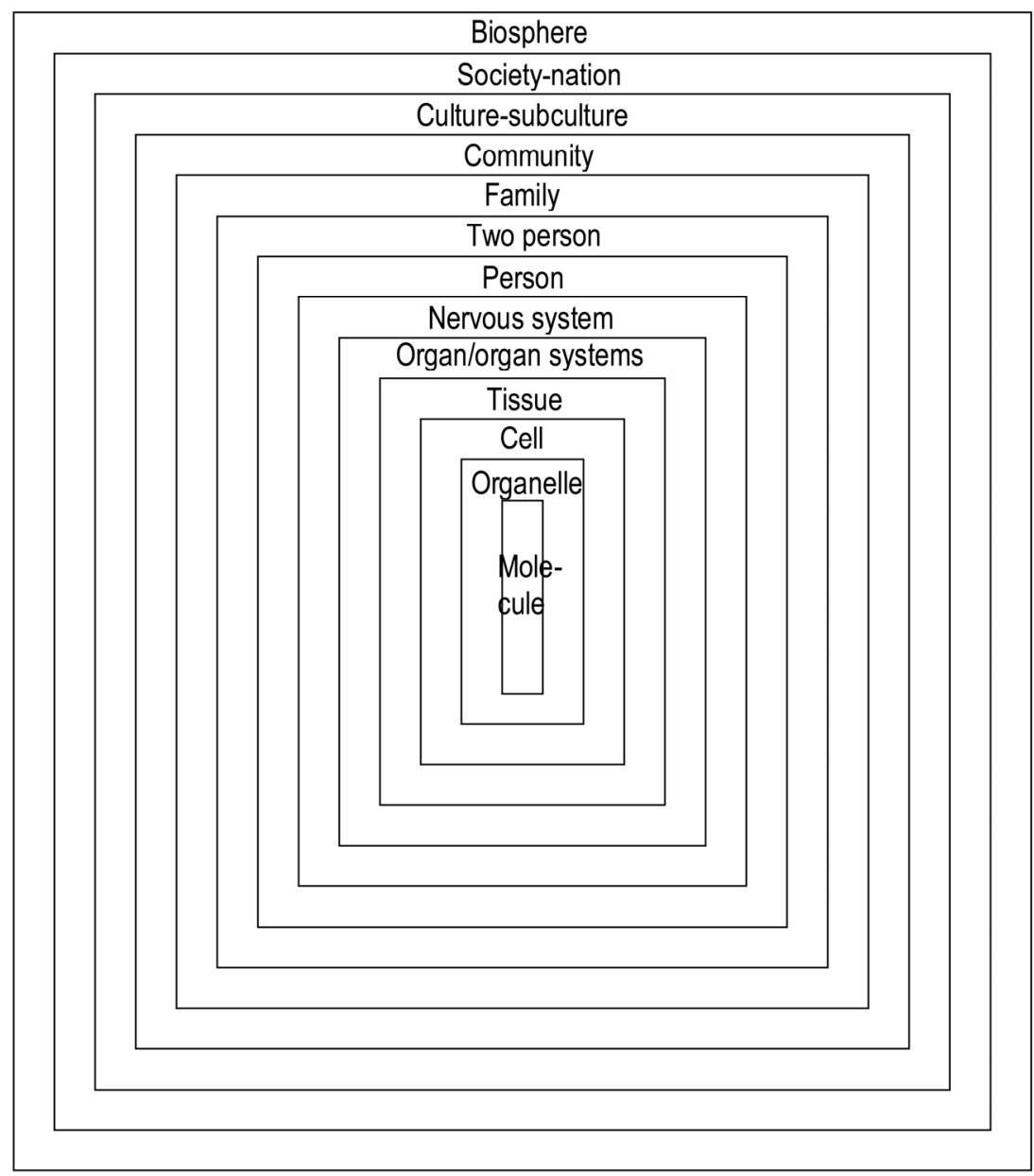

Figure 1. Biopsychosocial model based on Engel (1980)

\section{Social comparison, social support, and modeling}

Social comparison theory was fist formulated by Festinger (1954). In times of uncertainty, when other means for objective evaluation are lacking, individuals compare themselves to similar others to evaluate their own opinions and abilities. Social comparison may include both upward and downward comparisons (Stanton et al. 1999). Upward information and affiliation seeking with more fortunate others address motives for self- 
improvement, and downward comparisons with less fortunate others may serve ego-enhancement and positive affect maintenance (Taylor and Brown 1988). Some of the benefits from group interventions may be explained by comparison activities. Groups of fellow cancer survivors may provide both upward and downward social comparisons. For instance, group members displaying positive adjustment to cancer will be sought to provide models for self-improvement (upward contacts) and those whose physical health is poor may offer an opportunity for enhancing self-esteem and mood (downward evaluation) (Taylor and Lobel 1989).

Group interventions are also considered to provide opportunities for social support from peers, i.e. fellow-cancer patients. Helgeson and Cohen (1996) investigated the effectiveness of social support in group interventions among cancer patients. They distinguished emotional social support (by peers in peer discussion groups) and informational social support with regard to managing the illness (by professionals in educational groups). Educational groups aimed at providing informational support appeared to be as effective, if not more effective than, peer discussions for adjustment to cancer. The authors supposed that structured educational group interventions might be more effective than group discussion interventions, because they can offer in a structured manner both informational social support and emotional social support. Furthermore, they suggested that by providing group-wise information on the illness and by teaching ways to manage the illness and its side effects, feelings of control, self-esteem and optimism might be enhanced. A meta-analysis of psychological interventions to reduce cancer patients' anxiety and distress showed that psycho-educational group interventions were more effective than individual interventions (Sheard and Maguire 1999). Furthermore, a randomized controlled study in cancer patients showed that an educational group intervention had more long-term benefits for quality of life than a peer discussion intervention (Helgeson et al. 2001).

Modeling, or vicarious or observational learning is a concept in social cognitive theory (Bandura 1986). Bandura renamed social learning theory as social cognitive theory in his comprehensive framework for understanding human social behavior (Bandura 1986). Modeling means that a person may learn from the observation of behavior of others. For example, participants in group-based cancer rehabilitation may be able to watch the rehabilitation facilitators and their fellow group members engage in behavior they are learning. Observational learning is more efficient than operant conditioning for learning complex behaviors. The learning observer does not need to 
engage in the time-consuming trial-and-error process of operant conditioning, but discovers rules that account for the behavior of others by observing the behavior and the reinforcements they receive for their behavior. Models may show their self-regulatory behavior (Zimmerman 2000) and there is evidence that, for example, the persistence of a model during complex problem-solving affects positively the perseverance of observers (Zimmerman 2000). The positive effects of including social cognitive theory components, such as modeling, in psychosocial interventions for cancer patients were described in a meta-analysis (Graves 2003).

Acknowledging the biopsychosocial model (Engel 1977; Engel 1980; Engel 1997) and theory on social comparison (Bogart and Helgeson 2000; Festinger 1954; Stanton et al. 1999), social support (Helgeson and Cohen 1996) and modeling (Bandura 1986), "Recovery \& Stability" combined physical and psychosocial group interventions. This way, the program aimed to optimally improve the physical, psychological, and social domains of cancer survivors' quality of life (Aaronson et al. 1993; Ware and Sherbourne 1992).

\section{Theoretical foundation of "OncoRev"}

"OncoRev" preserved the multi-modal and group-wise approach that was used in "Recovery \& Stability", based on the theories outlined before. Furthermore, in addition to social comparison processes and modeling, other valuable concepts originating from social learning theory and social cognitive theory (Bandura 1986) were also applied. Self-efficacy (Bandura 1997), self-management (Holroyd and Creer 1986), and illness perceptions (Leventhal and Carr 2001) were addressed in both modules. Furthermore, problemsolving (D'Zurilla and Goldfried 1971; D’Zurilla and Nezu 2007) was integrated in the psychosocial module by formatting this module in line with existing and beneficial problem-solving protocols for individual cancer patients (Nezu et al. 1998; Nezu et al. 2003) and for groups of patients with nonspecific low back pain (Van den Hout et al. 2003; Kole-Snijders et al. 2006). Next, these concepts are described. 


\section{Social cognitive theory, self-efficacy, self-management, and illness perceptions}

Within social cognitive theory (Bandura 1986) human behavior is explained in terms of a triadic, dynamic and reciprocal model in which behavior, personal factors (including cognitions) and environmental influences all interact. Among the crucial personal factors are the individual's capabilities to symbolize behavior, to anticipate the outcomes of behavior, to learn by observing others, to have confidence in performing a behavior (including overcoming the problems in performing the behavior), to self-determine or self-regulate behavior, and to reflect on and analyze experience (Bandura 1997). A meta-analysis showed that when more social cognitive theory components were included in psychosocial interventions for cancer patients these interventions had greater positive effects on cancer patients' quality of life than interventions with no or fewer social cognitive theory components (Graves 2003). Behavior and the interpretation of its effect are shaped by modeling as well as by internal reflection and self-regulation (Bandura 1986). According to Bandura (1997) self-efficacy, or having confidence in the ability to perform a behavior, is the most important prerequisite for behavior change because it affects how much effort is invested in a given task and what level of performance is attained. Bandura defined self-efficacy as "belief in one's capabilities to organize and execute the course of action required to produce given attainments" (Bandura 1997) (p.3).

Self-management, or self-regulation, refers to the process of planning, monitoring and changing one's behaviors and cognitions to correspond with abilities, the environment, and desired outcomes. People are capable of selfregulation by influencing direct control over their own behavior and by selecting or changing environmental conditions that, in turn, influence their behavior. People adopt personal standards for behavior, appraise their behavior against such standards, and create unique incentives that motivate and guide their behavior (Maddux 1995). Like the biopsychosocial model, self-management is based on the assumption that people strive for maintaining homeostasis in a continuous process with cybernetic regulation mechanisms with positive and negative feedback loops. In health psychology selfmanagement refers to the performance of preventive or therapeutic health care activities, often in collaboration with health care professionals (Tobin et al. 1986). Self-management theory in chronic disease describes how patients cope with the consequences of their disease and their treatment by a circular process of goal selection, information collection, information processing and 
evaluation, decision-making, action and self-reaction (Creer 2000; Mesters et al. 2002). According to Creer (2000) the purpose of developing selfmanagement skills is that eventually the patient's attention will be less focused on his disease.

Illness perceptions play an important role in self-management. The basic assumption underlying the self-management approach is that people coping with cancer are "common sense medical scientists" (Leventhal and Carr 2001). Their formulation and representation of the illness guides their coping procedures, that is, their efforts in cancer rehabilitation (Leventhal and Carr 2001). According to the "common sense model of illness" coping strategies are guided by patients' perceptions of their illness (Leventhal and Carr 2001). Patients perceive their illness' identity (the abstract cognition or "label", and the concrete perceptions of the "symptoms"), causes, time-line (duration and course), consequences, controllability and curability. Therefore, awareness or even adaptation of these perceptions might influence coping and support self-management. A meta-analysis of different patient populations revealed that a stronger perception of identity, timeline and consequences was associated with passive coping and lower functioning (Hagger and Orbell 2003). In contrast, patients who perceived high controllability seemed to have more active coping styles and better functioning than those who perceived low controllability (Hagger and Orbell 2003). Therefore, rehabilitation should, for example, be aimed at increasing beliefs in personal control and changing beliefs about the course of illness and the consequences of the illness, to reach improvements in daily functioning.

\section{Problem-solving model of stress and coping}

The present problem-solving approach has its beginnings around 1970 and has developed since that time (D'Zurilla and Nezu 2007). Referring to social learning theory D'Zurilla and Goldfried first formulated problem-solving theory in 1971. According to the problem-solving model of stress and coping, problem-solving moderates the relations between stressful life events, like a cancer diagnosis and its aftermath, and distress, which ultimately influences a person's physical, psychological and social well-being (D'Zurilla and Nezu 2007; Nezu et al. 1998; Nezu 2004). Problem-solving is a general coping strategy, by which people manage and adapt to stressful life events as well as to related stressful daily problems and hassles, thereby enhancing their flexibility and perceived control, and minimizing emotional distress, even in situations that cannot be changed for the better (D'Zurilla 
and Nezu 2007; Nezu et al. 1998). Their coping efforts are aimed at changing the problematic nature of a situation itself, changing their reactions to such situations, or both. Thus, Lazarus' concepts of problem-focused and emotion-focused coping (Lazarus 1993) are both integrated in problemsolving theory (D'Zurilla and Nezu 2007). Problem-solving involves two processes. Firstly, problem orientation, positive or negative, is mainly a motivational process involving a person's generalized thoughts and feelings about problems and his own coping ability (D'Zurilla and Nezu 2007). Problem orientation is related to Lazarus' concepts of cognitive appraisal (primary and secondary) (Lazarus 1993) and Bandura's concepts of selfefficacy and outcome-expectancies (Bandura 1997; D'Zurilla and Nezu 2007). Secondly, problem-solving style, i.e. rational problem-solving, impulsive/carelessness style and avoidance style, refers to people's cognitive-behavioral activities aimed at finding solutions to particular problems (D'Zurilla and Nezu 2007). The circular problem-solving process, highly comparable to the self-management process, involves five steps: problem orientation, followed by systematically applying four rational problemsolving skills, i.e. problem definition and formulation (including goalsetting), generation of alternative solutions, decision making, and solution implementation and verification (D'Zurilla and Nezu 2007). In cancer patients, less effective problem-solving was related to more anxiety and depression and less quality of life. Particularly, more negative problem orientation was highly correlated with more anxiety and depression in cancer patients (Nezu et al. 1999b). Moreover, more negative problem orientation and less rational problem-solving predicted more anxiety and depression in cancer survivors years after medical treatment (Nezu et al. 1999b).

Problem-solving training for cancer patients aims to improve the patient's problem-solving, i.e. to enhance one's positive problem orientation, decrease one's negative problem orientation, increase one's rational problem-solving, and decrease one's impulsive/carelessness and avoidance styles, by cycling through the following steps (Nezu et al. 1998):

Problem orientation: adopt a positive and constructive orientation toward problems throughout one's life and, specifically, to problems due to cancer and its treatment. This includes: a) belief that it is common and normal to have these problems, b) belief that one is capable of improving his quality of life through effective problem-solving, c) reduce thoughts that might interfere with effective problem-solving (e.g. "I will fail, whatever I do") d) accept emotions (e.g. sadness), thoughts (e.g. worries), and physical symptoms (e.g. fatigue) as cues that a problem exists and as a signal to begin 
problem-solving, and e) use the "stop and think" method to stop responding to problems emotionally by either acting impulsively or avoiding dealing with the problem.

Problem definition and formulation: a) gather information about the problem, b) describe the facts in clear language, c) separate facts from assumptions, d) identify obstacles and conflicts, and e) set realistic goals.

Generation of alternative solutions/brainstorming: a) think of as many possible solutions as possible, b) defer judgment until later, and c) combine ideas.

Decision making: a) identify the consequences (long and short-term, regarding self and others), b) identify the consequences of alternative solutions and conduct a cost-benefit analysis, c) estimate the likelihood that a solution will successfully solve the problem, and d) estimate the likelihood that the implementation of a solution is practicable.

Solution implementation and verification: a) optimally carry out a solution plan, b) monitor the actual consequences of the implemented solution, c) evaluate whether the solution has solved the problem, d) engage in selfreinforcement if the problem is solved, and e) trouble shoot if the outcome is unsatisfactory and recycle the model if necessary.

This problem-solving training showed to have substantial short and longterm benefits for distressed cancer patients in problem-solving, distress and quality of life (Nezu et al. 2003). Furthermore, problem-solving training, by itself or as part of a larger treatment package, has been applied extensively for a variety of mental health problems (such as depression and anxiety) and medical conditions (including cancer, diabetes, back pain, arthritis, hypertension, headaches and obesity). Most studies of these interventions underscored the effectiveness of problem-solving training (D'Zurilla and Nezu 2007; Nezu 2004).

The multi-modal and group-wise approach in "Recovery \& Stability", based on the biopsychosocial model (Engel 1977; Engel 1980; Engel 1997) and concepts from social cognitive theory (Bandura 1986), was preserved in "OncoRev". The foundation for this refined program integrated concepts from social cognitive theory, i.e. self-efficacy (Bandura 1997), selfmanagement (Creer 2000) and illness perceptions (Leventhal and Carr 2001), and problem-solving theory (D'Zurilla and Nezu 2007). 


\section{Overview of the dissertation}

The quantitative study in chapter 2 and the qualitative study in chapter 3 focus on the multi-modal cancer rehabilitation program, "Recovery \& Stability". The quantitative studies in the chapters 4,5 and 6 focus on the multi-modal cancer rehabilitation program, "OncoRev". Chapter 2 presents a longitudinal cohort study evaluating the effects of "Recovery \& Stability" on quality of life. The focus group study in chapter 3 explored the views of former participants in "Recovery \& Stability" on how and to what extent the program had met their needs. This study describes their experiences during and after the program, their views on the value of cancer rehabilitation and their suggestions for improvement of the program. Chapter 4 contains a randomized controlled trial comparing short-term effects (up to 3-month follow-up) on cancer survivors' quality of life of combined comprehensive physical training and cognitive-behavioral psychosocial training, with comprehensive physical training. In this study, the two rehabilitation groups were compared with a waiting-list comparison group. The aim of the randomized controlled trial presented in chapter 5 was to compare the long-term effects up to 9-month follow-up on cancer survivors' quality of life of combined comprehensive physical and cognitive-behavioral psychosocial training, with comprehensive physical training. Chapter 6 presents a randomized controlled trial to investigate long-term effects up to 9-month follow-up on cancer survivors' problem-solving and distress of comprehensive physical training and the additional value of cognitive-behavioral psychosocial training. This study also investigated these effects in subgroups of participants with different pre-rehabilitation distress levels. Finally, chapter 7 provides a general discussion and conclusion. 


\section{Chapter 2}

\section{Quality of life of cancer survivors after physical and psychosocial rehabilitation}

Korstjens, I., Mesters, I., Van der Peet E., Gijsen B., \& Van den Borne B. (2006). Quality of life of cancer survivors after physical and psychosocial rehabilitation. European Journal of Cancer Prevention, 15, 541-547. 


\section{Abstract}

As the number of newly diagnosed cancer patients and the survival rates of cancer increase, more and more cancer patients are facing distressing physical and psychosocial problems as a result of their cancer and its treatment. To address these problems, a 12-week rehabilitation group program for cancer patients (all cancer types), combining physical exercise and psycho-education, was tested in a longitudinal cohort study $(\mathrm{n}=658)$. At baseline, participants reported a low quality of life, measured by the European Organization for Research and Treatment of Cancer (EORTC) Quality of Life Questionnaire (QLQ-C30) (range 0 - 100). Halfway through the intervention significant improvements were found in all domains, except cognitive functioning. At the end of rehabilitation, participants had reached significant improvements on all outcome variables: in global quality of life (from 63.0 to 71.9), emotional functioning (from 66.2 to 74.4), cognitive functioning (from 70.5 to 74.4 ) and fatigue level (reduction from 49.8 to 41.8) while, moreover, non-breast cancer patients showed clinically relevant improvement in physical functioning (from 67.3 to 80.4 ) and social functioning (63.4 to 79.4 ) and non-working patients showed a clinically relevant improvement in role functioning (from 57.0 to 69.6). 


\section{Introduction}

Throughout the world cancer is diagnosed in more than ten million persons each year and the World Health Organization has predicted that this number may increase by $50 \%$ in the next twenty years (Parkin et al. 2001). Furthermore, survival rates after cancer increase which means that, presently, cancer must be managed as a chronic disease. Many cancer survivors who have completed their medical treatment are facing distressing physical and psychosocial problems as a result of their illness and its treatment. These physical difficulties (e.g. fatigue, decreased physical capacity) and psychosocial problems (e.g. anxiety, depression, stress, insecurity, grief, decreased self-esteem, hindered job reintegration, social isolation) lead to diminished quality of life (Curt et al. 2000; Ganz et al. 1996; Gotay et al. 2002; Tomich and Helgeson 2002). Many physical interventions (Courneya and Friedenreich 1999; Irwin and Ainsworth 2004; Oldervoll et al. 2004; Schwartz 2004) and psychosocial interventions (Andersen 2002; Fawzy et al. 1995; Meyer and Mark 1995) have been developed for cancer patients during treatment and survivors who have completed medical treatment. Reviews of these studies show benefits of both physical and psychosocial interventions on physical functioning, psychosocial functioning and quality of life. Reviewing psychological interventions for cancer patients, Andersen (2002) found that the focus has expanded from primarily reducing stress and enhancing quality of life to biobehavioral change (e.g. health behaviors, biological responses, disease outcomes), while a recent review of physical activity after a cancer diagnosis explicitly focused on the psychosocial outcomes of exercise as well (Schwartz 2004). Noticing this trend in looking more broadly at mechanisms and effects in cancer rehabilitation and acknowledging the value of Engel's bio-psychosocial model (Engel 1977; Engel 1997) it can be postulated that combining physical and psychosocial interventions in multi-modal cancer rehabilitation may enhance improvements in quality of life beyond the benefits of a single focused intervention. So far in only very few studies physical and psychosocial interventions have been combined (Berglund et al. 1994; Courneya et al. 2003a; Mock et al. 1994; Van Weert et al. 2004). Although sample sizes in these evaluation studies were small (14 - 199) and though there were differences in study design, patient characteristics, interventions and outcome measures, these studies suggest that a combined rehabilitation program can be beneficial in a mixed group of patients and might enhance improvements in quality of life beyond the benefits of a single intervention. Based on this principle of 
combining physical exercise and psycho-education, in 1996 a 12-week rehabilitation group program has been developed and implemented to improve quality of life of cancer survivors who have completed their medical treatment. In this paper the results are presented of a longitudinal cohort study $(n=658)$ evaluating this program. We hypothesized that this program would lead to an improvement in different dimensions of quality of life with a special focus on the decrease of fatigue.

\section{Methods}

\section{Rehabilitation program}

The aim of this group program was to enhance quality of life and recovery among cancer survivors (all cancer types). The program included a strong physical fitness component and a psycho-educational component. The group format provided opportunities for social comparison (Bogart and Helgeson 2000; Festinger 1954; Stanton et al. 1999) social support (Helgeson and Cohen 1996) and modeling (Bandura 1986). The program took 3 months, during which groups of $12-16$ participants visited the rehabilitation center for physical training and psycho-education. The contents of the program were described in a protocol. The physical training module followed a fairly detailed instruction for each session, whereas in the psycho-education module the protocol determined for each session the theme and the health care professionals and provided, in broad outlines, instructions for these sessions.

Physical training was provided for 2 hours twice a week under the guidance of two expert physiotherapists, and was aimed at improving movement skills, strength and endurance, coping with physical complaints like fatigue, enhancing feelings of control and stress reduction. Each physical training session consisted of individual training of endurance and strength (60 minutes) or a group sports and games program (60 minutes), combined with aqua aerobics in an indoor swimming pool (30 minutes). Each physical training session included a break for refreshments and mutual contact and sharing experiences among the participants. The training was based on graded activity theory (Vlaeyen et al. 1993). Individual training on bicycle ergo-meters and fitness apparatus was started at a low level and was gradually build up, following an individualized training scheme based on among 
others age, body height, weight, sub-maximal strength tests (e.g. leg curl, lat pull, rowing, abdominal, bench press, leg extension, abductor, peck deck, adductor), sit- and reach test, and sub-maximal endurance tests (submaximal bicycle exercise Astrand Test). Each session in the sports and games program had a central theme, like capability and cooperation, throwing and catching, balance, coordination, social contact, winning and losing, and relaxation. These sessions consisted of warming up, main exercises and cooling down, performed individually, in a duo or in a group. The aqua aerobics sessions in the swimming pool followed the same structure and included themes like jogging, walking and jumping, pulling and pushing, relay race and ballgames.

Psycho-education consisted of seven 2-hour sessions (midday or evening) and was aimed at providing support in coping with cancer and enhancing self-confidence and autonomy. During the sessions participants were provided with information on cancer-related subjects and were given opportunity to share their experiences as cancer survivors. The evening sessions enabled partners and close family members to join in some of the sessions. Different health professionals familiar with oncology (oncology nurse, psychologist, social worker, dietary advisor) led the meetings on subjects like life changes and cancer, communicating about cancer, nutrition and cancer, coping with stress, creative emotional expression, insurances issues and returning to work, and lymph-oedema and skin care.

\section{Study design}

A longitudinal cohort design was applied in which participants filled out the Quality of Life Questionnaire of the European Organization for Research and Treatment of Cancer (EORTC QLQ C-30) at baseline, again at week 6 (halfway) and at week 12 (end of the program) (Aaronson et al. 1993).

\section{Recruitment and participants}

The program was developed for adults with a diagnosis of cancer (all cancer types), who had completed their primary treatment (surgery, radiotherapy, chemotherapy, immunotherapy) preferably at least 2 months ago, and who experienced a discrepancy between their present and their optimal level of functioning with complaints concerning physical restrictions (such as reduced capacity, decreased level of activities, increased fatigue), and/or psychological and social restrictions (such as emotional instability, anxiety, 
depression, nervousness, sleep disturbances), and were physically, psychologically and socially capable of participating in a group program. Participants needed to be referred by their medical specialists or general practitioners and were excluded if they were physically at risk due to the cancer or serious co-morbidity, or in case of serious psychopathology, serious cognitive disturbances, restricting side-effects of medication, or if they needed more complex rehabilitation.

Still, despite these selection criteria and due to the unpredictable character of their disease, participants could be confronted with recurrence or a new diagnosis of cancer during the program.

All 665 patients who participated in the program gave their consent to take part in the evaluation of the program. Dropout and reasons for dropout from the program were not recorded. Participants whose data were collected neither at baseline (7 patients) nor at the following measurements (halfway through the program accumulating to 24 patients; final assessment 86 patients) were counted as measurement dropout. Thus, as questionnaires were filled in at the rehabilitation centre, it appeared that 658 patients started rehabilitation, and that halfway through the program $634(96 \%)$ and at the end of the program still 579 (87\%) participated in the study.

\section{Measurement instruments}

The EORTC QLQ-C30 is a 30 item self-report questionnaire for cancer patients, which incorporates a global quality of life scale ( 2 items, $\alpha=0.80$ ), five functional scales: physical functioning ( 5 items, $\alpha=0.44$ ), role functioning ( 2 items, $\alpha=0.79$ ), cognitive functioning ( 2 items, $\alpha=0.68$ ), emotional functioning ( 4 items, $\alpha=0.87$ ) and social functioning ( 2 items, $\alpha$ $=0.76$ ), and three symptom scales: fatigue ( 3 items, $\alpha=0.82$ ), pain ( 2 items, $\alpha=0.84$ ) and nausea and vomiting ( 2 items, $\alpha=0.66$ ). The remaining six single items assess additional symptoms commonly reported by cancer patients: dyspnea, appetite loss, insomnia, constipation and diarrhea and financial difficulties. The EORTC QLQ-C30 is a reliable and valid instrument that has been used in many studies evaluating clinical and psychosocial interventions with cancer patients (Aaronson et al. 1993; Apolone et al. 1998; King 1996; McLachlan et al. 1998).

In this study only the global scale, the functional scales and the fatigue symptom scale were assessed since participants were not under treatment for cancer anymore and because fatigue is a serious and frequently reported complaint after treatment as well (Curt et al. 2000; Servaes et al. 2002). 
After applying a linear transformation procedure according to the EORTC QLQ C-30 manual, the scores of the scales ranged from 0 to 100, a higher score reflecting a higher quality of life at the global and functional scales and a higher level of symptoms at the symptom scale. Although the internal consistency of the physical functioning subscale was not optimal, this subscale was still applied for comparison with results from other studies. The inter-item correlations of the other scales ranged from adequate to good (Nunnally 1970).

Background variables were gender (male/female), age (in years), interaction gender/age, marital status (married/not married), employment before diagnosis (yes/no), working at start rehabilitation (yes/no), children (yes/no), children living at home (yes/no), cancer type (breast cancer/other cancers), treatment (radiotherapy/operation/chemotherapy), time since diagnosis (in months), renewed confrontation through recurrence or new diagnosis (yes/no), and kinesiophobia. Kinesiophobia as measured with the Tampa scale, assessed excessive, irrational and debilitating fear of physical movement and activity resulting from a feeling of vulnerability to painful injury or re-injury (Goubert et al. 2004). Factor-analysis revealed two subscales: avoidance of activities ( 7 items, $\alpha=0.68$, range 7 - 28) and pathologic somatic focus (four items, $\alpha=0.71$, range 4 - 16).

\section{Statistical analysis}

Statistical analysis was performed using the Statistical Package for the Social Sciences (SPSS Release 11.5 2002). Nonparametric Wilcoxon Signed Ranks Tests were used to determine effects on quality of life halfway (T0T1) and at the end of rehabilitation (T0-T2). Multiple Linear Regression analyses (enter method) were conducted to assess the influence of background variables on the improvements of quality of life halfway (T0-T1) and at the end (T0-T2). Similar analyses were conducted to determine the influence of these same factors (controlling for quality of life at the start) on quality of life halfway and at the end of rehabilitation. Thus for each domain of quality of life which was assessed in this study (seven subscales) four Multiple Linear Regression analyses were conducted. If background variables in these analyses were found to determine significantly quality of life $(p<0.05)$ halfway or at the end of rehabilitation, participants were split in two subgroups on the basis of these variables. Subsequently, Wilcoxon Signed Ranks Tests were conducted for each subgroup separately. If nonparametric Mann-Whitney Tests confirmed significant differences between 
these subgroups $(p<0.05)$ results were reported for each subgroup (Table 2). Intention-to-treat analyses, following the "last observation carried forward" method, were carried out on all outcome variables.

\section{Results}

\section{Patient characteristics}

Baseline measurements were obtained from 658 participants (period 19972002).

Table 1. Patients' characteristics at start of rehabilitation (T0) $n=658$

\begin{tabular}{|c|c|c|c|}
\hline Age in years & & Cancer type \% & \\
\hline Mean (SD) & $50.6(9.5)$ & Breast & 54.3 \\
\hline Range & $18-75$ & Blood/lymphoma & 11.4 \\
\hline Missing \% & 6.1 & Digestive tract & 7.3 \\
\hline Gender \% & & Gynaecologic & 6.4 \\
\hline Male & 21.0 & Lung & 5.8 \\
\hline Female & 77.8 & Urologic & 4.1 \\
\hline Missing & 1.2 & Rest & 3.3 \\
\hline Marital Status \% & & Missing & 7.4 \\
\hline Married/living together & 77.7 & Treatment \% & \\
\hline Not married/single & 16.9 & Operation & 14.4 \\
\hline Missing & 5.5 & Chemotherapy & 6.7 \\
\hline Children \% & & Radiotherapy & 1.1 \\
\hline Yes & 76.9 & Operation/chemo & 20.8 \\
\hline No & 17.8 & Operation/radio & 20.3 \\
\hline Missing & 5.3 & Chemo/radio & 4.1 \\
\hline At home & 37.7 & Operation/chemo/radio & 27.8 \\
\hline Not at home & 44.8 & Missing & 4.8 \\
\hline Missing & 17.5 & Time since diagnosis in years & \\
\hline Employment status \% & & Mean (SD) & $2.1(2.8)$ \\
\hline Employed at diagnosis & 48.3 & Range & $0-25$ \\
\hline Not employed at diagnosis & 45.2 & Missing \% & 9.0 \\
\hline Missing & 6.5 & Time since end of treatment in years & \\
\hline Working at start & 15.8 & Mean (SD) & $1.3(2)$ \\
\hline Not working at start & 71.0 & Range & $0-14$ \\
\hline Missing & 13.2 & Missing \% & 10.8 \\
\hline \multicolumn{4}{|l|}{ TAMPA kinesiophobia score } \\
\hline Avoidance of activities & $13.0(3.9)$ & & \\
\hline Pathologic somatic focus & $9.0(3.1)$ & & \\
\hline Missing \% & 3.6 & & \\
\hline
\end{tabular}

Abbreviation: SD: standard deviation. 
Participants were predominantly female (Table 1) and the majority was married or lived together. Most patients had children and about one third had children living at home. About half of the participants were employed at the time of diagnosis, but at the start of rehabilitation only one fifth was actually at work.

Half of the participants were diagnosed with breast cancer. The other participants were known with a mix of cancer types, among which the largest group consisted of blood and lymphoma cancer patients. Most participants $(83.3 \%)$ had undergone surgery, and often this therapy was combined with chemotherapy and/or radiotherapy (68.9\%). About 13\% had been treated with chemotherapy only, radiotherapy only or a combination of these two. Most patients entered rehabilitation within one year after the end of their medical treatment.

\section{Effects on quality of life}

Table 2 presents the short and long term effects on quality of life $(n=658)$. If an effect was significantly modified by a background variable the results are presented for the subgroups based on this background variable. For example cancer type was a significant effect modifier in the long term effects in physical functioning and social functioning: therefore these results are presented separately for breast cancer patients and other cancer patients. To allow comparison with other studies limited to breast cancer patients, Table 2 also shows for all subscales separate information for breast cancer patients and other cancer patients (n, mean, SD). EORTC reference values for all subscales are also included. 
Table 2. Short and long-term effects on quality of life (EORTC QLQ-C30) (range 0 - 100) $(n=658)$

\begin{tabular}{|c|c|c|c|c|c|c|c|c|c|c|}
\hline & \multirow[t]{2}{*}{ Time } & \multirow[t]{2}{*}{ Effect modifier } & \multirow[t]{2}{*}{$\mathrm{n}$} & \multirow[t]{2}{*}{ Mean (SD) } & \multirow{2}{*}{$\begin{array}{l}\text { Wilcoxon } \\
\text { Signed Ranks } \\
\text { Test }\end{array}$} & \multicolumn{2}{|c|}{ Breast cancer } & \multicolumn{2}{|c|}{ Other cancers } & \multirow{2}{*}{$\begin{array}{l}\text { EORTC } \\
\text { reference } \\
\text { values }\end{array}$} \\
\hline & & & & & & $n$ & $\begin{array}{l}\text { Mean } \\
(\mathrm{SD})\end{array}$ & $\mathrm{n}$ & $\begin{array}{l}\text { Mean } \\
\text { (SD) }\end{array}$ & \\
\hline \multirow{4}{*}{$\begin{array}{l}\text { Global quality } \\
\text { of life }\end{array}$} & T0-T1 & - & 649 & $63.0(17.8)^{* \star *}$ & -5.073 & 354 & $65.3(16.3)$ & 259 & $60.2(19.3)$ & \multirow{4}{*}{$\begin{array}{l}66.3^{a} \\
73.4^{b} \\
75.3^{c}\end{array}$} \\
\hline & & & 593 & $67.5(18.0)$ & & 334 & $69.4(17.4)$ & 226 & $65.3(18.6)$ & \\
\hline & T0-T2 & - & 649 & $63.0(17.8)^{* * *}$ & -9.422 & 354 & $65.3(16.3)$ & 259 & $60.2(19.3)$ & \\
\hline & & & 554 & $71.9(17.3)$ & & 323 & $73.1(17.5)$ & 201 & 70.7 (16.7) & \\
\hline \multirow{7}{*}{$\begin{array}{l}\text { Physical } \\
\text { functioning }\end{array}$} & T0-T1 & - & 647 & $69.9(18.2)^{* \star *}$ & -6.679 & 352 & $71.1(16.4)$ & 259 & $67.3(20.2)$ & \multirow{6}{*}{$\begin{array}{l}73.6^{a} \\
85.7^{b} \\
89.9^{c}\end{array}$} \\
\hline & & & 592 & $75.9(17.9)$ & & 333 & 76.0 (17.1) & 226 & $76.2(19.0)$ & \\
\hline & \multirow[t]{4}{*}{ T0-T2 } & Breast cancer & 352 & $71.1(16.4)^{* * *}$ & -5.812 & & & & & \\
\hline & & & 325 & $77.2(17.4)$ & & & & & & \\
\hline & & Other cancers & 259 & $67.3(20.2)^{* * *}$ & -7.247 & & & & & \\
\hline & & & 201 & $80.4(18.8)+$ & & & & & & \\
\hline & \multirow[t]{2}{*}{ T0-T1 } & - & 649 & $58.5(25.5)^{\star * *}$ & -6.415 & 354 & $61.1(24.8)$ & 259 & $55.5(26.7)$ & \multirow{6}{*}{$\begin{array}{l}76.6^{a} \\
84.6^{b} \\
83.3^{c}\end{array}$} \\
\hline \multirow{5}{*}{$\begin{array}{l}\text { Role } \\
\text { functioning }\end{array}$} & & & 592 & $67.0(23.3)$ & & 333 & $69.0(23.0)$ & 226 & $65.6(23.7)$ & \\
\hline & \multirow[t]{4}{*}{ T0-T2 } & Working at T0 & 104 & $68.3(21.2)$ n.s. & -1.686 & 65 & $69.5(19.7)$ & 35 & $67.1(24.4)$ & \\
\hline & & & 93 & $72.8(22.8)$ & & 61 & $73.0(20.7)$ & 28 & $75.6(24.2)$ & \\
\hline & & Not working at & 458 & $57.0(25.5)^{* * *}$ & -7.739 & 253 & $59.6(24.9)$ & 188 & $53.7(26.1)$ & \\
\hline & & TO & 392 & $69.6(24.7)+$ & & 231 & $70.2(24.3)$ & 144 & $69.9(25.0)$ & \\
\hline \multirow{7}{*}{$\begin{array}{l}\text { Emotional } \\
\text { functioning }\end{array}$} & \multirow[t]{5}{*}{ T0-T1 } & Pathologic & 351 & $72.2(23.1)^{* *}$ & -2.765 & 208 & $73.2(22.5)$ & 123 & $70.5(24.3)$ & \multirow{7}{*}{$\begin{array}{l}67.3^{a} \\
70.4^{b} \\
82.2^{c}\end{array}$} \\
\hline & & somatic focus & 321 & $75.4(22.7)$ & & 190 & $75.8(21.7)$ & 113 & $75.0(24.6)$ & \\
\hline & & at $\mathrm{T} 0 \leq 9$ & & & & & & & & \\
\hline & & Pathologic & 283 & $59.4(24.7)^{* * *}$ & -4.323 & 139 & $58.8(23.4)$ & 129 & $59.6(26.3)$ & \\
\hline & & $\begin{array}{l}\text { somatic focus } \\
\text { at TO }>9\end{array}$ & 253 & $67.0(22.7)$ & & 132 & $66.4(24.2)$ & 108 & $68.0(21.1)$ & \\
\hline & \multirow[t]{2}{*}{ T0-T2 } & \multirow[t]{2}{*}{-} & 648 & $66.2(24.8)^{* \star *}$ & -6.396 & 354 & $67.2(24.1)$ & 258 & $64.5(26.1)$ & \\
\hline & & & 553 & $74.4(21.7)$ & & 323 & $74.1(22.7)$ & 200 & $74.9(20.7)$ & \\
\hline \multirow{4}{*}{$\begin{array}{l}\text { Cognitive } \\
\text { functioning }\end{array}$} & \multirow[t]{2}{*}{$\mathrm{T} 0-\mathrm{T} 1$} & - & 647 & 70.5 (26.1) n.s. & -0.054 & 354 & $70.9(26.9)$ & 257 & $70.1(25.3)$ & $83.1^{\mathrm{a}}$ \\
\hline & & & 591 & $71.5(24.7)$ & & 332 & $72.1(24.1)$ & 226 & 71.5 (25.1) & $85.6^{b}$ \\
\hline & T0-T2 & - & 647 & $70.5(26.1)^{\star *}$ & -2.836 & 354 & $71.1(27.1)$ & 257 & $70.1(25.3)$ & $86.5^{c}$ \\
\hline & & & 554 & $74.4(24.3)$ & & 323 & $74.0(24.6)$ & 201 & $75.6(23.6)$ & \\
\hline Social & T0-T1 & - & 646 & $68.6(27.5)^{* * *}$ & -6.785 & 353 & $72.6(25.2)$ & 257 & $63.4(29.5)$ & $77.3^{a}$ \\
\hline functioning & & & 591 & $77.8(24.1)$ & & 332 & $80.4(23.0)$ & 226 & 74.6 (25.2) & $86.4^{b}$ \\
\hline & T0-T2 & Breast cancer & 353 & $72.7(25.2) * * *$ & -5.035 & & & & & $85.8^{c}$ \\
\hline & & & 323 & $80.0(22.4)$ & & & & & & \\
\hline & & Other cancers & 257 & $63.4(29.5)^{* * *}$ & -6.429 & & & & & \\
\hline & & & 201 & $79.4(22.9)+$ & & & & & & \\
\hline Fatigue & T0-T1 & - & 646 & $49.8(25.3)^{* *}$ & -3.469 & 353 & $48.7(25.0)$ & 257 & $51.0(26.2)$ & $31.4^{a}$ \\
\hline & & & 592 & $45.4(24.6)$ & & 333 & $44.6(25.1)$ & 226 & $45.5(24.4)$ & $20.6^{b}$ \\
\hline & T0-T2 & - & 646 & $49.8(25.3)^{\star * *}$ & -6.397 & 353 & $48.7(25.0)$ & 257 & $51.0(26.2)$ & $28.8^{c}$ \\
\hline & & & 556 & $41.8(25.0)$ & & 325 & $41.0(25.1)$ & 201 & $43.0(25.5)$ & \\
\hline
\end{tabular}


Abbreviations: SD: standard deviation; EORTC QLQ-C30: European Organization for Research and Treatment of Cancer Quality of life Questionnaire. T0: baseline; T1: halfway program, T2: end of program. For comparisons, additional information on breast cancer and other cancers ( $n$, mean, SD), and EORTC reference values. Reference scores EORTC Data Center (2003) (EORTC, 2003):

a All breast cancer patients $(n=1217)$ (local: $n=197$, regional: $n=341$, advanced cancer: $n=679$ );

b Local and local regional breast cancer patients $(n=538)$ (local: $n=197$, regional cancer: $n=341)$;

c General Norwegian population random sample $(n=1965$ (male: 1016, female 949).

${ }^{*} p<0.05,{ }^{* *} p<0.01,{ }^{* * *} p<0.001,+d>10$ (clinically relevant difference) (King, 1996).

Table 2 shows that global quality of life had improved significantly halfway (posttest T1) and at the end of the program (posttest T2) compared with the start of the intervention period. At subscale level the following results were found. All posttest physical functioning scores had improved significantly compared with the pretest scores and, even more importantly, at the end of rehabilitation improvements appeared to be clinically relevant $(\mathrm{d}>10)$ for persons with a non-breast cancer diagnosis (who started initially at a significantly lower score than the breast cancer patients). At both $\mathrm{T} 1$ and $\mathrm{T} 2$ role functioning improved significantly, but apparently merely the non- working population (who started at a lower level than the working population) contributed significantly to the overall improvement (T0-T2), which again was also clinically relevant for the non-working participants. Emotional functioning significantly improved as well at both posttest moments. During the period T0-T1 persons who scored high on pathologic somatic focus improved relatively more in emotional functioning compared with persons with a low score on this variable. Improvements on cognitive functioning were only significant at the last posttest measurement. Both halfway and at the end of the intervention period participants improved on social functioning. Again, especially the non-breast cancer groups progressed most. For them, at the end of rehabilitation the improvements were not only significant, but also clinically relevant. Fatigue decreased significantly both halfway and at the end of the program. No discrepancies were found between the results presented in Table 2 and the results of intention-to-treat analyses, except that $\mathrm{T} 0-\mathrm{T} 2$ changes in intention-to-treat analyses were no longer clinically relevant $(>10)$ for non-breast cancer patients in physical functioning (8.4) and for non-working participants in role functioning (9.6). In Table 2 we included EORTC reference values (EORTC 2003) to compare the quality of life scores of our participants with other relevant groups of cancer patients and the general population. At the start of rehabilitation participants functioned worse in all quality of life domains compared to the reference group of all breast cancer patients. Halfway (T1) participants reported better global quality of life and physical and social functioning than 
this reference group and furthermore patients with low pathologic somatic focus even showed better emotional functioning than the reference group of local and local regional breast cancer patients. At the end of rehabilitation participants still performed worse in role functioning and cognitive functioning and showed more fatigue, but reported better global quality of life and physical and social functioning as compared to the reference group of all breast cancer patients. Moreover, they reported better emotional functioning than the patients with local and local regional breast cancers at the end of rehabilitation.

\section{Discussion}

\section{Main findings}

Firstly, after 6 weeks of rehabilitation, participants reported significant improvements in global quality of life, various types of functioning (physical functioning, role functioning, emotional functioning and social functioning), as well as significantly reduced fatigue. Secondly, after 12 weeks participants had further progressed in all domains (cognitive functioning was also significantly improved) and by then clinically relevant improvements were found in social functioning for non-breast cancer patients. These patients also showed clinically relevant improvement in physical functioning (but not in intention-to-treat analyses) and non-working participants showed clinically relevant improvement in role functioning (but not in intention-to-treat analyses). Thirdly, we found that most of our background variables were not significantly related to quality of life and that the effects of other background variables were limited: at $\mathrm{T} 1$ only pathologic somatic focus in emotional functioning, at T2 cancer type in physical and in social functioning and at T2 employment in role functioning. These results show that this multi-focus rehabilitation program had positive effects in all domains of quality of life for a mixed group of cancer survivors and they show that the 12-week program was more adequate than a 6 -week program. In addition, the small measurement dropout rates ( $4 \%$ halfway and $13 \%$ after twelve weeks) while dropout due to recurrence or a new diagnosis of cancer is inevitable, indicate that participants were highly motivated and that a 12-week program was feasible. The sample size of our study almost doubles the sample sizes of the four combined rehabilitations programs we found so far (Berglund et al. 1994; Courneya et al. 2003a; Mock et al. 1994; Van Weert et al. 2004). 
Taken together, these studies focused predominantly on breast cancer patients, as is the case in most cancer survivor studies, while almost half of our study population were other cancer patients. As outcome measures in these studies were all different from our quality of life assessment, it is hard to make comparisons. Still, as we found positive effects in all domains of quality of life, no differences between breast cancer patients and other cancer patients in most domains and even better results for the latter in physical and in social functioning, we can add to the findings of these studies that a rehabilitation program for a mixed group of cancer patients is beneficial and feasible.

Compared with the EORTC reference values of cancer patients the participants in our study reported worse scores in all quality of life domains at baseline, which indicates that the program is followed by cancer survivors who are experiencing physical and psychosocial problems and who most are in need of professional support. Moreover, Berglund et al. (1997) found that invited eligible cancer patients who declined to take part in their rehabilitation program showed lower problem levels than participants. In a quantitative and qualitative assessment of rehabilitation needs in cancer patients $26 \%$ of the study population wanted to receive professional help, largely determined by perceived quality of life and social support, and these patients would prefer a rehabilitation program focusing on fatigue, reinforcing physical working capacity and psychosocial functioning (Van Harten et al. 1998). These findings indicate that rehabilitation is predominantly needed by patients with low quality of life and supports the need to tailor rehabilitation to the specific physical and psychosocial needs of these participants.

\section{Limitations of study and recommendations for future research}

The first limitation of this study is that it was impossible to determine whether changes in the different domains of quality of life were a result of the exercise, the psycho-education, or a combination of both. A study in which each of these components is treated individually would be necessary to assess the separate impacts. The second limitation was the lack of a comparison group, which made it impossible to determine whether the improvements in quality of life resulted from the rehabilitation program or were an effect of natural restoration after the primary therapy. Although the consistency of significant improvements in all domains might indicate that the improvements might well be an effect of the rehabilitation program a dismantling study should also include a waiting list control group. Thirdly, 
long-term effects were not assessed in this study and should be measured in future research. The fourth limitation was that our study focused mainly on quality of life. In daily functioning this is the most important outcome for patients in cancer rehabilitation but, in order to be able to understand the mechanisms that determine quality of life, future research should incorporate objective rigorous physical strength and endurance tests and validated measurement instruments for more specific psychosocial parameters such as self-efficacy, coping skills, social support, anxiety and depression. A process evaluation would also be needed, consisting of a detailed protocol of the rehabilitation program and rigorous validity and fidelity checks.

To date, the rehabilitation program we studied is being applied in more than 30 centers, in the Netherlands and Belgium. Currently, an adapted version of this program is tested in a multi-center dismantling trial, comparing the combined program with a physical program and a waiting list control group, and 9 months of follow-up. 


\section{Chapter 3}

\section{Cancer patients' view on rehabilitation and quality of life: a program audit}

Korstjens, I., Mesters, I., Gijsen, B., \& Van den Borne B. (2008). Cancer patients' view on rehabilitation and quality of life: a programme audit. European Journal of Cancer Rehabilitation, 7, 290-297. DOI: 10.1111/j.1365-2354.2007.00864.x 


\section{Abstract}

An existing 12-week cancer rehabilitation group program, combining physical training and psychosocial sessions, was recently tested in a quantitative longitudinal cohort study $(n=658)$ among adult patients who have problems coping with cancer. Results showed improvements in all quality of life domains. The objective of the present study was to explore patients' perspectives on how and to what extent the rehabilitation program had met their needs in order to optimize the program. Patients who participated in the program no longer than five years ago were interviewed in three focus groups $(n=23)$. Participants saw rehabilitation as provided by the program as an important steppingstone in their ongoing recovery process and identified the integrated physical training, psychosocial sessions and patient-topatient contacts as a powerful and supportive combination. Recommendations for improvements to the program mainly concerned the psychosocial module. Information needs varied strongly among participants and, in this respect, it was hard to satisfy all of them. Participants suggested that the program should focus more on developing skills to cope with cancer, its consequences, and emerging problems after rehabilitation. In conclusion, participants valued the group-based multi-modal cancer rehabilitation program and recommended more focus on developing coping skills. 


\section{Introduction}

Because survival rates of cancer increase (Parkin et al. 2001), cancer must be managed more and more as a chronic disease, which calls for structured selfmanagement interventions. Reviews of separate physical interventions (Courneya and Friedenreich 1999; Irwin and Ainsworth 2004; Oldervoll et al. 2004; Schwartz 2004) and psychosocial interventions (Andersen 2002; Fawzy et al. 1995; Meyer and Mark 1995) for cancer patients show benefits on physical and psychosocial functioning, and other quality of life domains. Several combined cancer rehabilitation interventions (Berglund et al. 1994; Courneya et al. 2003a; Mock et al. 1994; Korstjens 2006b; Van Weert et al. 2004; Van Weert et al. 2005) showed beneficial quantitative outcomes. To understand the working mechanisms in such a combined program qualitative data were collected from participants in a 12-week multi-modal cancer rehabilitation program that showed significant improvements in quality of life (Korstjens et al. 2006b). This article describes participants' experiences in this multi-modal program, their views on the value of cancer rehabilitation and their suggestions for improvement of the program.

\section{Rehabilitation program}

The program was based on a biopsychosocial approach (Engel 1977; Engel 1980; Engel 1997). It combined physical fitness and psychosocial activities in a group format to provide opportunities for social comparison (Bogart and Helgeson 2000; Festinger 1954; Stanton et al. 1999), social support Helgeson and Cohen 1996) and modeling (Bandura 1986). During 3 months, groups of $12-16$ participants (all cancer types, primary medical treatment at least 2 months ago) visited a rehabilitation center. Physical training followed a fairly detailed protocol for each session, whereas in the psychosocial module the manual determined for each session the theme and health care professionals and provided in broad outlines instructions for these sessions.

Physical training was based on graded activity theory (Vlayen et al. 1993) and consisted of 2-hour sessions, twice a week, under guidance of two expert physiotherapists. The training was aimed at improving movement skills, strength and endurance, coping with physical complaints like fatigue, enhancing feelings of control and stress reduction. Included were exercises on bicycle ergo-meters and fitness apparatus, starting at a low level and gradually building up, following an individualized training scheme, based on 
strength and endurance tests. Each session included a refreshment break with opportunity for mutual contact and sharing experiences among the participants. Sports and games, walking outdoors, aqua-fitness and relaxation exercises completed physical training.

Psychosocial sessions included seven 2-hour sessions (midday or evening) to provide support in coping with cancer and enhancing self-confidence and autonomy. Participants received information on cancer-related subjects and could share their experiences as cancer survivors. Partners were also invited in order to involve them in the program and to support them in their coping with the disease. Various professionals familiar with oncology (oncology nurse, psychologist, social worker, dietary advisor) led the meetings on subjects like life changes and cancer, communicating about cancer, nutrition and cancer, coping with stress, creative emotional expression (e.g. painting), insurances issues and returning to work, and lymph-oedema and skin care. Generally, these sessions consisted of lectures with opportunity for posing questions, and psychological exercises (e.g. creative emotional expression) were sometimes included.

\section{Method}

\section{Design}

Focus group interviews were conducted to explore participants' experiences and perspectives on how and to what extent the rehabilitation program had met their needs related to physical and psychosocial functioning.

\section{Recruitment and participants}

Patients $(\mathrm{n}=250)$ who participated in the rehabilitation program no longer than five years ago (in 1998, 2000 and 2002) received a written invitation to the focus groups as part of continuous quality monitoring, adaptation, and development of the ongoing rehabilitation program. Attached to the invitation potential participants received written information from the researchers about the focus group study, an informed consent form and a short questionnaire on demographic details and basic information about their illness and rehabilitation. It was emphasized that participation was strictly voluntary. Participants could choose between several dates within a 2-week period and 
three locations for the interviews. Sixty-nine participants returned the informed consent form and were able and willing to participate at a specified time-point and location. Finally, 23 participants who participated in the program during $1998(\mathrm{n}=5), 2000(\mathrm{n}=10)$ and $2002(\mathrm{n}=8)$ could be scheduled for one of three focus groups. Participants in groups $1(n=4)$ and $2(\mathrm{n}=9)$ all were rehabilitated in the same center, while group $3(\mathrm{n}=10)$ consisted of participants from the other center.

\section{The focus group process}

In all interviews the same format was applied, according to principles and guidelines in conducting focus groups (Morgan and Krueger 1998). Three authors, independent researchers and experienced in conducting focus groups in cancer patients, moderated the interviews. Each interview was guided by two moderators. Discussions were recorded on audiotapes with the permission of the participants. Each interview lasted approximately 2 hours. A five-topic guide was used to promote focus during the interviews. Main themes were the influence of the rehabilitation program and of its components as perceived by the participants, most valued components, expectations about the rehabilitation program, and experiences after the rehabilitation program. For each topic specific open-ended conversational worded questions were formulated by the authors to obtain some uniformity in how questions were asked in different focus groups. Identification of questions resulted from cancer rehabilitation literature in general, site visit observations of the first author and from the effect-study data of the program in which the focus group participants were involved. To check validity and applicability during the interviews the questions were orally pre-tested for comprehensibility, simplicity and clarity in health care researchers and in non-researchers. After a short introduction of the focus group process by the moderators the discussion was focused on the themes in the topic guide. The moderators stressed their neutrality by exploring both positive and negative remarks of participants. At the conclusion of each interview the moderators invited participants to provide feedback on the discussion and to verify a short oral summary. Directly after an interview the moderators discussed their findings and formulated areas that called for more in depth exploration in the next focus group interview. Eventually, participants were invited to comment on a mailed summary of the focus group findings. At this point no further comments were returned. 


\section{Analysis}

The audiotaped discussions in Dutch were transcribed verbatim. Transcripts were loaded into QSR NVivo Revision 1.3 for categorization and coding. Data were categorized on the basis of background literature, research questions and the data itself. Categories were restructured and refined by means of sequential and retrospective searching of the transcripts. The data were then compared and contrasted within and among interviews and eight key themes (derived from 21 sub-themes) appearing throughout all interviews were identified (Morgan and Krueger 1998). Quotes were translated into English by the first author and retranslated by the second author. The qualitative findings from the focus group interviews are presented as descriptive summaries and interpretations of the eight key themes, supported and illustrated by quotes from the raw data. Before each quote the IDnumber of the focus group is given.

Nonparametric Chi-Square Tests for nominal data and Mann Whitney Tests for interval data were used to test for differences between baseline characteristics of the focus group participants and the effect study population. For these analyses we combined two datasets; the effect study dataset, including those participants who were also recruited for the focus groups, and the focus group dataset (Korstjens et al 2006b).

\section{Results}

\section{Participants' characteristics}

No statistical significant differences were found between the characteristics at the start of rehabilitation of the focus groups participants $(n=23)$ and the effect study participants $(n=658)$ (Table 1$)$. So, the focus group participants represented the effect study population in baseline characteristics. Most focus group participants were female (91.3\%) and were married or living together (95.7\%). The majority was employed during diagnosis $(69.6 \%)$, but still not working at the start of rehabilitation (65.2\%). Most participants were diagnosed with breast cancer (73.9\%). Almost all participants had undergone an operation $(91.3 \%)$, often combined with chemotherapy or radiotherapy $(78.3 \%)$. 
Table 1. Characteristics of participants in focus groups $(n=23)$ and of participants in effect study $(n=658)$ at start of rehabilitation

\begin{tabular}{|c|c|c|c|c|c|c|c|}
\hline \multirow{2}{*}{$\begin{array}{l}\text { Characteristics } \\
\text { Age in years }\end{array}$} & \multicolumn{2}{|c|}{$\begin{array}{l}\text { Focus } \\
\text { groups }\end{array}$} & \multirow[t]{2}{*}{$\begin{array}{l}\text { Effect } \\
\text { study }\end{array}$} & \multirow{2}{*}{$\begin{array}{l}\text { Characteristics } \\
\text { Cancer type } \mathrm{n} \text { and \% }\end{array}$} & \multicolumn{2}{|c|}{$\begin{array}{l}\text { Focus } \\
\text { groups }\end{array}$} & \multirow[t]{2}{*}{$\begin{array}{l}\text { Effect } \\
\text { study }\end{array}$} \\
\hline & & & & & & & \\
\hline Mean (SD) & \multicolumn{2}{|c|}{$51.6(6.5)$} & $50.6(9.5)$ & Breast & 17 & 73.9 & 54.3 \\
\hline Range & \multicolumn{2}{|c|}{$38-67$} & $18-75$ & Blood/lymphoma & 3 & 13.0 & 11.4 \\
\hline Missing \% & \multicolumn{2}{|l|}{-} & 6.1 & Digestive tract & - & - & 7.3 \\
\hline \multicolumn{3}{|l|}{ Gender $\mathrm{n}$ and $\%$} & & Gynecologic & 3 & 13.0 & 6.4 \\
\hline Male & 2 & 8.7 & 21.0 & Lung & - & - & 5.8 \\
\hline Female & 21 & 91.3 & 77.8 & Urologic & - & - & 4.1 \\
\hline Missing & - & - & 1.2 & Rest & - & - & 3.3 \\
\hline \multicolumn{4}{|l|}{ Marital Status $n$ and $\%$} & Missing & - & - & 7.4 \\
\hline $\begin{array}{l}\text { Married/living } \\
\text { together }\end{array}$ & 22 & 95.7 & 77.7 & \multicolumn{4}{|l|}{ Treatment $\mathrm{n}$ and $\%$} \\
\hline Not married/single & 1 & 4.3 & 16.9 & Operation & 3 & 13.0 & 14.4 \\
\hline Missing & - & - & 5.5 & Chemotherapy & 1 & 4.3 & 6.7 \\
\hline \multicolumn{4}{|l|}{ Children $\mathrm{n}$ and $\%$} & Radiotherapy & - & - & 1.1 \\
\hline Yes & 18 & 78.3 & 76.9 & Operation/chemo & 5 & 21.7 & 20.8 \\
\hline No & 5 & 21.7 & 17.8 & Operation/radio & 7 & 30.4 & 20.3 \\
\hline Missing & - & - & 5.3 & Chemo/radio & 1 & 4.3 & 4.1 \\
\hline At home & 8 & 34.8 & 37.7 & Operation/chemo/radio & 6 & 26.1 & 27.8 \\
\hline Not at home & 13 & 56.5 & 44.8 & Missing & - & - & 4.8 \\
\hline Missing & 2 & 8.7 & 17.5 & \multicolumn{4}{|c|}{ Time since diagnosis in years } \\
\hline \multicolumn{4}{|c|}{ Employment status $\mathrm{n}$ and \% } & Mean (SD) & \multicolumn{2}{|c|}{$1.8(1.8)$} & $2.1(2.8)$ \\
\hline $\begin{array}{l}\text { Employed at } \\
\text { diagnosis }\end{array}$ & 16 & 69.6 & 48.3 & Range & \multicolumn{2}{|c|}{$0-6$} & $0-25$ \\
\hline $\begin{array}{l}\text { Not employed at } \\
\text { diagnosis }\end{array}$ & 7 & 30.4 & 45.2 & Missing \% & \multicolumn{2}{|l|}{-} & 9.0 \\
\hline Missing & - & - & 6.5 & \multicolumn{4}{|c|}{ Time since end of treatment in years } \\
\hline Working at start & 7 & 30.4 & 15.8 & Mean (SD) & & $(1.7)$ & $1.3(2)$ \\
\hline Not working at start & 15 & 65.2 & 71.0 & Range & 0 & & $0-14$ \\
\hline Missing & 1 & 4.3 & 13.2 & Missing \% & - & & 10.8 \\
\hline
\end{tabular}

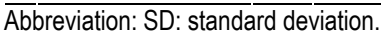

\section{Qualitative findings}

The headings of the next sections represent the eight key themes that were derived from the focus group interviews.

\section{Expectations about rehabilitation program}

Participants heard about the rehabilitation program for the first time from their doctors, family, friends, colleagues, local television, or read leaflets in 
hospitals. However, several participants stressed that many other cancer patients still seemed unaware of the existence of the program. They stated that information about the program should be part of regular cancer care to enable more patients to enter this kind of rehabilitation. Other patients had hesitated about signing in, because they were not convinced of their need for rehabilitation and dreaded being confronted with other patients' experiences. Expectations were diverse and often rather vague, but most participants expected a group program in which they had to exercise in the first place. Most participants saw rehabilitation as a new start after the intensive period of medical treatment.

F3: I needed the program to recover physically. I used to exercise a lot, but when I got ill I almost couldn't do anything anymore. But, I did not dare to go to my old sports clubs. I just needed a safe environment... . to find out what I could still do and to build it up again in a supportive and safe environment... I found that the group and the mutual contacts with other patients were very pleasant and important, although that was not my reason for entering.

\section{Influence of rehabilitation on quality of life}

Participants mentioned that first of all the disease itself had changed their view on life in both negative (e.g. uncertainty, fear for recurrence) and positive ways (e.g. sense of perspective, more joy in life). Most participants stated that their quality of life had improved by rehabilitation as a whole and they did not distinguish separate effects of the two main program components, but, sometimes, psychological improvement was specifically attributed to physical change.

F3: Afterwards, . . not only my physical condition had improved but my mental state as well. When you become stronger physically, your mind also gets stronger. That's very important to recover and put you back on track in life.

Participants almost unanimously said that their physical condition had improved and also regaining confidence in body functioning was considered important. Former frequent sportswomen appreciated that at the start of rehabilitation all the participants shared a rather low physical capacity, or at least their capacity was not as high as in regular sports clubs. 
F3: There it's all about performing and you don't just shout from the rooftops: "Guys, I'm taking it easy for a while". Because, when you are tired . . . you could see them thinking: "Gee, she can't keep up anymore". That's what makes you feel worse. So you start doing less and less, because you keep being confronted with yourself. This program helps you start over from scratch.

After rehabilitation some participants (re-) entered regular sports clubs or physical training groups especially for former cancer rehabilitators. Many patients had experienced unpredictable and persisting fatigue due to cancer and its treatment, influencing many domains of life (e.g. cognitive and emotional functioning, and social activities). Although most patients experienced less fatigue after the program, and became more energetic and capable of engaging in more activities, for many of them fatigue remained to be dealt with.

F1: In dealing with fatigue I found my own rhythm. For example, I've made a day schedule and do something for 30 minutes and the next 30 minutes I take a rest. . . That makes me feel fitter.

Most participants felt psychosocial improvements. They had regained selfconfidence, felt stronger and more assertive. Often they mentioned that rehabilitation had helped them to integrate their experiences and the consequences of cancer in their lives.

F3: Working with a group has helped me to give cancer a place, to accept it in a way and to accept my limits . . . Now I think: "I'll never be the same person that I used to be". That's how you learn to accept your situation and look at things differently, to prioritize and divide tasks in parts ... It gives me more energy to do the things I like to do.

Some participants remarked that they became more self-assured and more open to others.

F1: I do feel that it's easier for me to open up and tell other people that I had it myself.

Still, in the long run, many participants experience cancer as an ongoing threat. 
F1: I think that you've got a small scar on your soul that will always stay with you. And you keep being confronted with that again and again. If you feel just a teensy weensy little thing: "Oh please, don't let it be...".

\section{Group format}

Almost all participants considered it important to rehabilitate in a group of fellow cancer patients. It was mentioned that males were a minority, especially by male focus group participants, but this was not experienced as disturbing. Differences were noticed in age, cancer type, physical capacity, and time since diagnosis, but these all seemed to be less important than supporting each other by sharing experiences, worries, humor, and striving for physical and mental improvement.

F3: Mutual contacts with fellow patients . . you don't have to explain the fear you felt, the uncertainty, the grief. Everyone felt the same. That really felt good.

For some it was helpful to swim together and to show their body in public again after cancer and the treatment they had.

F3: Imagine what you look like after both your breasts have been amputated and you put on your swimsuit, without prostheses. But here nobody would stare at you. You'd just dive into the pool and not be too afraid to say: "Look, I've got a new swimsuit!". At some point you could even be proud of yourself.

However, being confronted with other patients' problems can be hard too.

F2: I found it very hard. Everyone around me had some complaint or other and then someone got ill again... I just didn't want to have anything to do with it anymore.

Still, these inevitable confrontations helped to evaluate one's situation, led to downward comparisons (e.g. "I am better of") as well as upward comparisons (e.g. "She does not give up either") with fellow patients. Also fellow patients served as models in coping with cancer. 
F2: Before I came here I thought: "I am nothing, there is no "me" anymore". Then I met all these people here and I heard how ill they have been. I saw how they talked and felt about it, and still managed to laugh and have a good time together. That actually helped me get over it.

\section{Composition of rehabilitation program}

Participants considered the duration of the program feasible and adequate to enable them to make a new start.

F3: A steppingstone. It's about defeating your weakness; you need to do that in steps. You can't just go back to your old life. This offered a safe environment where I could experiment.

Most participants appreciated the program as a whole; even if at the start of rehabilitation they were more attracted to physical training than to the psychosocial module.

F2: And there I was: down in the dumps ... I refused to see a psychiatrist, but I knew I had to do something . . for me, the program as a whole was very positive: the psychosocial sessions, the contacts with fellow patients, the exercise. I think it actually did have a positive influence on me, because it forced me to cope with it, to face up to myself. And I was forced to talk about it, although I didn't want to do that at al. But now I believe it was good that they made me talk about it.

Evaluation of physical training sessions

Physical training was almost unanimously considered very important and helpful in building up physical capacity. Due to individualized training schemes and individual guidance by professional therapists participants did not feel hindered by differences in physical capacity. Physical training was also perceived as helpful in regaining mental strength and flexibility, and especially the value of group sports and swimming was mentioned, e.g. in overcoming fear and regaining self-confidence.

F1: These people were throwing the ball and I just didn't do that anymore . . I I didn't think I could do that, but then I thought: well, these people can do it and they have experienced the same thing . . that felt 
so good! . . Everything I did made me feel really scared. And the exercise made me forget all that. So, I just joined in.

Also some participants put things in perspective, recognizing that not only cancer, but also getting older means that one has to adjust and has to learn to cope with one's limits.

F2: I still experience fatigue . . It's different from being tired after exercising, which feels kind of nice. But I've learned to cope with that. I wouldn't have learned that anywhere else.

\section{Evaluation of psychosocial sessions}

Compared to physical training, experiences and opinions concerning psychosocial sessions were much more diverse. The attendance rate of rehabilitators, as well as their partners, at these sessions had differed considerably between rehabilitation groups. Also over the years there were changes in sequence and scheduling of psychosocial sessions (at the rehabilitation center or elsewhere, during the day or in the evening) and in session leaders. Finally, many groups followed these sessions in the same formation as during physical training, which supported group cohesion, while in other cases patients from different groups were brought together during psychosocial sessions. These differences may have caused variation in appreciation. In the first place there was much variation in participants' needs and in the appreciation of the amount and sort of information they received. Some participants recognized that it was very difficult for course leaders to deliver information that satisfied all.

F3: It strongly depends on the composition of the group and the phase in the illness process in which the various group members find themselves. What's relevant for "fresh" patients differs from what's relevant for "experienced" patients. Additionally, a group, consisting of older people that are no longer working is very different from a group consisting of mainly young people, where other problems also play a role. .. It's also influenced by the person who guides the session.

On the other hand, receiving and exchanging information also showed to serve other goals, like facilitating the sharing of experiences. 
F2: So actually the psychosocial sessions offered an occasion to get together and than the talking just seemed to happen all by itself. But if someone is pushing you to talk then and there, I don't know if that would work for me.

Some session leaders facilitated restructuring as well.

F3: The session focusing on coping with stress, which was guided by a communication expert, was very very good. She made us put our thoughts down on paper ... to get things straight in our mind. In short, she offered tools for structuring thing ... If there is just sharing of experiences, like some session leaders did . . . that leaves you without enough structure . . . The communication expert did that very well, she gave everyone enough opportunity to talk, and pointed to the connecting thread in our stories as we went along.

It appeared that, contrary to the physical module, the psychosocial module was experienced rather as loosely connected themes, delivered by various session leaders in different ways.

Participants' recommendations for improvements to the program

Regarding the physical module one participant recommended to also pay attention to complaints that people might not experience during rehabilitation, but that were to be expected afterwards, when they took up new tasks again. Suggestions for improvements to the program mainly focused on the psychosocial module. Many participants mentioned themes that should be skipped or adjusted (e.g. nutrition and cancer, lymph-oedema and skin care, insurances issues and returning to work). Others missed themes like heredity, sexual relations, family relations, especially with (younger) children, and fatigue. A few participants suggested inviting an oncologist (for information about cancer, treatment and consequences, or communication about patientdoctor relations) or to invite cancer survivors for information on long-term survival experiences. One remark was made repeatedly: the need for more coaching in getting "a grip on life" after cancer. In physical training, next to rebuilding physical capacity, learning to cope with limited capacities and fatigue was highly valued. Therapists had paid attention to this throughout the module, but in the psychosocial module there were limited opportunities for continuous guidance in developing skills to deal with cancer, its conse- 
quences, recurrences (in yourself or others), survival, and the future. Often this was felt as a lack.

F1: When I got cancer, I spent a lot of time thinking about my life, and I discovered that I had done many things just because I'd been raised that way. Suddenly I realized, I have to set boundaries, but because I'd never done that, it's something I have to learn. That's where coaching is needed, and that should be stressed more . . . with a psychologist whom you can ask things and who can advise you. Now, I had to find that out myself.

F3: I would have very much liked to see a psychologist or a psychiatrist as part of the program . . . about the psychosocial effects of having cancer, about one's personality before and after cancer, and how to get something positive out of it. Some people say: having cancer and coming out of it positive, that's not possible. But it is! The question is: how?

Overall, participants indicated that the psychosocial module, in analogy to the physical module, needed more continuity and structure, and several participants suggested that a connecting thread could be a psychologist coaching them in skills to cope with many different aspects of the cancer experience.

Most valued aspects of rehabilitation program

In the first place, the mutual contacts with other fellow patients were perceived as the most valuable aspect of rehabilitation.

F1: The mere contact with fellow patients was very valuable. They understand what you are dealing with. Everyone understands you, your grief, your disappointment.

Physical training was seen as valuable, because it facilitated building up physical capacity in a safe context and at the same time facilitated mutual contacts and sharing of experiences. 


\section{Discussion}

Participants saw cancer rehabilitation as a valuable step in an ongoing process towards recovery. They perceived the 3-month duration of the program as adequate and feasible. Several patients mentioned that in regular cancer care patients should be informed about the program and the opportunities for participation. Although participants differed beforehand in their goals and expectations about the components of the program, afterwards, they were almost unanimous in their appreciation of group-wise integrated physical and psychosocial training. The mutual contacts with fellow cancer patients were seen as most valuable. Although many participants felt that these contacts were unsettling, much more so, they were experienced as helpful in overcoming physical as well as mental barriers. Mutual contacts were promoted during both the physical module (e.g. performing sports activities together) and the psychosocial module (e.g. sharing experiences). The physical module was also appreciated because of the individual and continuous guidance by professional therapists and because most participants experienced both improved physical capacity as well as improved coping with limited physical capacities and fatigue. Opinions about the psychosocial module were more mixed. Information and opportunities for sharing and dealing with experiences were valued, but some participants stressed that information did not always fit to individual needs and some participants had wished to receive more continuous and structured coaching in coping with cancer and its consequences.

The positive quantitative outcomes of the program's biopsychosocial approach (Korstjens et al. 2006b) were confirmed by the generally positive experiences of participants who appreciated the multi-modal program. The sample size of this study $(\mathrm{n}=23)$ was small compared with the cohort $(\mathrm{n}=$ 658) of the quantitative effect study, but it reflected the baseline characteristics rather well. It cannot be ruled out that selection and response bias occurred, resulting from the most able and willing, and possibly most satisfied rehabilitators entering the study. However, in the invitation the moderators explicitly stressed that as independent researchers they aimed to reach a full understanding of the views of the participants and the focus group discussion did provide recommendations for improvements to the program.

Overall, participants indicated that they would value a cancer rehabilitation group program in which systematic attention is paid to individual physical and psychosocial needs and which provides structured guidance in develop- 
ing skills to get "a grip on their life" again. In analogy to the physical protocol, a more structured psychosocial protocol with weekly sessions, guided by the same therapists throughout the module, might further enhance the group process and support participants more in their individual information and coaching needs. Information could then be focused on a few relevant topics for everyone (like stress and fatigue), with references to other information channels, while emphasis could be put on developing selfmanagement skills and applying them to individual needs. The focus group interviews were conducted as part of continuous quality monitoring, adaptation, and development of the ongoing rehabilitation program. To date, the rehabilitation program is being applied in more than 50 centers in the Netherlands and Belgium and is becoming part of regular cancer care. Based on findings in our quantitative study (Korstjens et al. 2006b) and this focus group study the program was further adapted. The highly valued aspects were maintained, such as the group format and multi-modal rehabilitation, while in both modules more emphasis is placed on self-management. This adapted program is presently being tested in a multi-center dismantling trial, comparing multi-modal rehabilitation to physical training and to no intervention.

In conclusion, participants valued group-based multi-modal cancer rehabilitation as an important steppingstone in their ongoing recovery process and recommended to improve the program by focusing more on developing coping skills. 


\section{Chapter 4}

\section{Quality of life after self-management cancer rehabilitation: a randomized controlled trial comparing physical and cognitive- behavioral training versus physical training}

Korstjens, I., May, A.M., Van Weert, E., Mesters, I., Tan F., Ros, W.J.G., Hoekstra-Weebers, J.E.H.M., Van der Schans, C.P., \& Van den Borne, B. (2008). Quality of life after selfmanagement cancer rehabilitation: a randomized controlled trial comparing physical and cognitive-behavioral training versus physical training. Psychosomatic Medicine, 70, 422-9. DOI: 10.1097/PSY.0b013e31816e038f. 


\section{Abstract}

Objective. To conduct a randomized controlled trial and compare the effects on cancer survivors' quality of life in a 12-week group-based multidisciplinary self-management rehabilitation program, combining physical training (twice weekly) and cognitive-behavioral training (once weekly) with those of a 12-week group-based physical training (twice weekly). In addition, both interventions were compared to no intervention.

Methods. Participants (all cancer types, medical treatment completed at least 3 months ago) were randomly assigned to multidisciplinary rehabilitation $(\mathrm{n}=76)$ or physical training $(\mathrm{n}=71)$. The nonintervention comparison group consisted of 62 patients on a waiting-list. Quality of life was measured using the RAND-36. The rehabilitation groups were measured at baseline, after rehabilitation, and 3-month follow-up, and the non intervention group was measured at baseline and 12 weeks later.

Results. The effects of multidisciplinary rehabilitation did not outperform those of physical training in role limitations due to emotional problem (primary outcome) or any other domains of quality of life (all $\mathrm{p}>0.05$ ). Compared with no intervention, participants in both rehabilitation groups showed significant and clinically relevant improvements in role limitations due to physical problem (primary outcome; effect size $(E S)=0.66$ ), and in physical functioning $(\mathrm{ES}=0.48)$, vitality $(\mathrm{ES}=0.54)$ and health change (ES $=0.76)($ all $\mathrm{p}<0.01)$.

Conclusions. Adding a cognitive-behavioral training to group-based self-management physical training did not have additional beneficial effects on cancer survivors' quality of life. Compared with the nonintervention, the group-based self-management rehabilitation improved cancer survivors' quality of life. 


\section{Introduction}

Because survival rates of cancer are growing (Parkin et al. 2001) cancer must be managed as a chronic disease. Approximately $26 \%$ of cancer survivors report decreased quality of life (QoL) and express a need for professional support in managing physical and psychosocial problems following cancer diagnosis and its treatment (Van Harten et al. 1998). Metaanalyses of randomized controlled trials of physical interventions (McNeely at al. 2006; Schmitz et al 2005) or psychosocial interventions (Rehse and Pukrop 2003) Meyer and Mark 1995) for cancer patients report small-tolarge effect sizes (ES) (range 0.28 - 0.84) for QoL. Moreover, a metaanalysis of randomized controlled studies of cognitive-behavioral interventions in cancer survivors reported large effects (ES 1.45) on QoL (Osborn et al. 2006). QoL is a multidimensional construct that includes physical as well as psychosocial dimensions. Therefore, acknowledging the biopsychosocial model (Engel 1997), combined physical and psychosocial interventions may enhance effects on QoL beyond those of single focused interventions. Indeed, combined interventions have also shown positive effects in cancer patients during medical treatment (Mock et al. 1994; Rummans et al. 2006), and after medical treatment (Berglund et al. 1994; Courneya et al. 2003a; Korstjens 2006b; Van Weert et al. 2005). Yet, no ES were reported of these interventions compared to no intervention. To date, only one study has compared a combined intervention to a single focused intervention (Courneya et al. 2003a). In that study, the combination of group psychotherapy and home-based walking as compared with group-psychotherapy alone improved QoL in two domains; functional well-being and fatigue. This finding is consistent with other studies in cancer survivor groups, suggesting that exercise is associated primarily with the physical and functional aspects of QoL, rather than the social and emotional dimensions (Karvinen et al. 2007) and may have the most benefits in physical and functional domains (Courneya et al. 2003b). The goal of multidisciplinary cancer rehabilitation is to improve participants' functioning in their daily roles by reducing physical as well as emotional problems. Therefore, we expect that adding a cognitive-behavioral intervention to physical training (PT) in multidisciplinary rehabilitation might show improvements in QoL beyond those of PT, primarily in role functioning associated with emotional problems. The present four-center trial is the first study to compare combined physical and cognitive-behavioral training $(\mathrm{PT}+\mathrm{CBT})$ to $\mathrm{PT}$ in cancer survivors. Furthermore, this is the first study to include a 3-month follow-up of cancer patients 
in a combined intervention as compared with a single focused intervention. Additionally, we compare the rehabilitation groups (PT and $\mathrm{PT}+\mathrm{CBT}$ combined) to a waiting list comparison group (WLC). To this end, we developed a multidisciplinary cancer rehabilitation program, based on earlier studies concerning multidisciplinary rehabilitation (Korstjens et al. 2006b; Van Weert et al. 2005). In this program, we integrated evidence-based (McNeely et al. 2006; Meyer and Mark 1995; Osborn et al. 2006; Rehse and Pukrop 2003; Schmitz et al. 2005) PT and CBT protocols according to a self-management approach (Leventhal and Carr 2001; Mesters et al. 2002). PT focused on enhancing self-management in physical training and sports, while in CBT emphasis was placed on providing structural interactive psycho-education and training in self-management skills. First, we hypothesized that $\mathrm{PT}+\mathrm{CBT}$ would demonstrate more improvement in QoL than PT after rehabilitation and at 3-month follow-up, primarily in the domain of role limitations due emotional problem. Second, we hypothesized that after rehabilitation both rehabilitation groups would outperform WLC, primarily in reducing role limitations due to physical problem.

\section{Methods}

\section{Study design}

In this prospective controlled four-center study, participants were randomly assigned to $\mathrm{PT}+\mathrm{CBT}$ or $\mathrm{PT}$, whereas participants awaiting rehabilitation elsewhere were assigned to WLC. Measurements were performed before and after rehabilitation (12 weeks after baseline), and at 3-month follow-up (6 months after baseline) in PT+CBT and PT. Participants in WLC filled out questionnaires at baseline and 12 weeks later (post-test).

\section{Settings and participants}

The centers involved were: two university medical centers, one general hospital and one rehabilitation center, all of them located in the Netherlands. The medical ethics committee of the University Medical Center Utrecht and the local research ethics committees approved the study. Written informed consent was obtained from each participant. Eligible for the study were cancer patients (age $\geq 18$ years) who completed curative cancer treatment at least 3 months ago, with a minimum estimated life expectancy of 1 year. 
They needed to be referred by a medical specialist or a general practitioner who then judged fulfillment of at least three of the following criteria: physical complaints, reduced physical capacity, psychological problems, increased fatigue, sleep disturbances, and problems in coping with reduced physical and psychosocial functioning due to cancer. Patients were excluded in case of restrictive side effects from medication, serious cognitive disturbances, psychopathology, or emotional instability that might impede participation in rehabilitation, or if they needed intensive medical treatment or rehabilitation. So, cancer survivors were eligible for the study when they were considered to be successfully treated for cancer, but still experienced physical and psychosocial problems.

\section{Recruitment and allocation}

Leaflets handed out by general practitioners or by medical professionals in hospitals, local newspapers, radio and television, and a website (www.oncorev.nl) informed cancer patients about the study. Patients who contacted a study center received a study information package, containing an information letter, an informed consent form, an intake questionnaire and referral papers for their medical specialists or general practitioners. Each center delivered one group at a time. In each center, consecutive groups of 8 - 12 eligible subjects were assigned to $\mathrm{PT}+\mathrm{CBT}$ or PT and were scheduled for baseline measurements. Randomization at group level was applied; before enrolling participants in the study, the sequence of PT $+\mathrm{CBT}$ and PT groups at each center was determined by an independent researcher from the University Medical Center Utrecht using a randomization list. After randomization, the sequence of treatments in each center was fixed and could not be influenced by research centers or researchers. Until the first session, participants were blinded to the rehabilitation group they were allocated to. Center $1(\mathrm{n}=15)$ delivered one PT $+\mathrm{CBT}$ and one PT group, centers $2(\mathrm{n}=$ $44)$ and $3(n=39)$ each delivered two PT + CBT and two PT groups, and center $4(\mathrm{n}=49)$ delivered three PT $+\mathrm{CBT}$ and three PT groups. Eligible patients were invited to participate in WLC if they had to wait at least 3 months for 12-week group-based multidisciplinary cancer rehabilitation programs in other Dutch centers using the same inclusion criteria. This population was expected to be highly comparable to participants in the rehabilitation groups. Interested WLC patients received the study information package and, after written consent, filled out questionnaires. 


\section{Recruitment and assessments of participants occurred between February 2004 and September 2006 (Figure 1).}

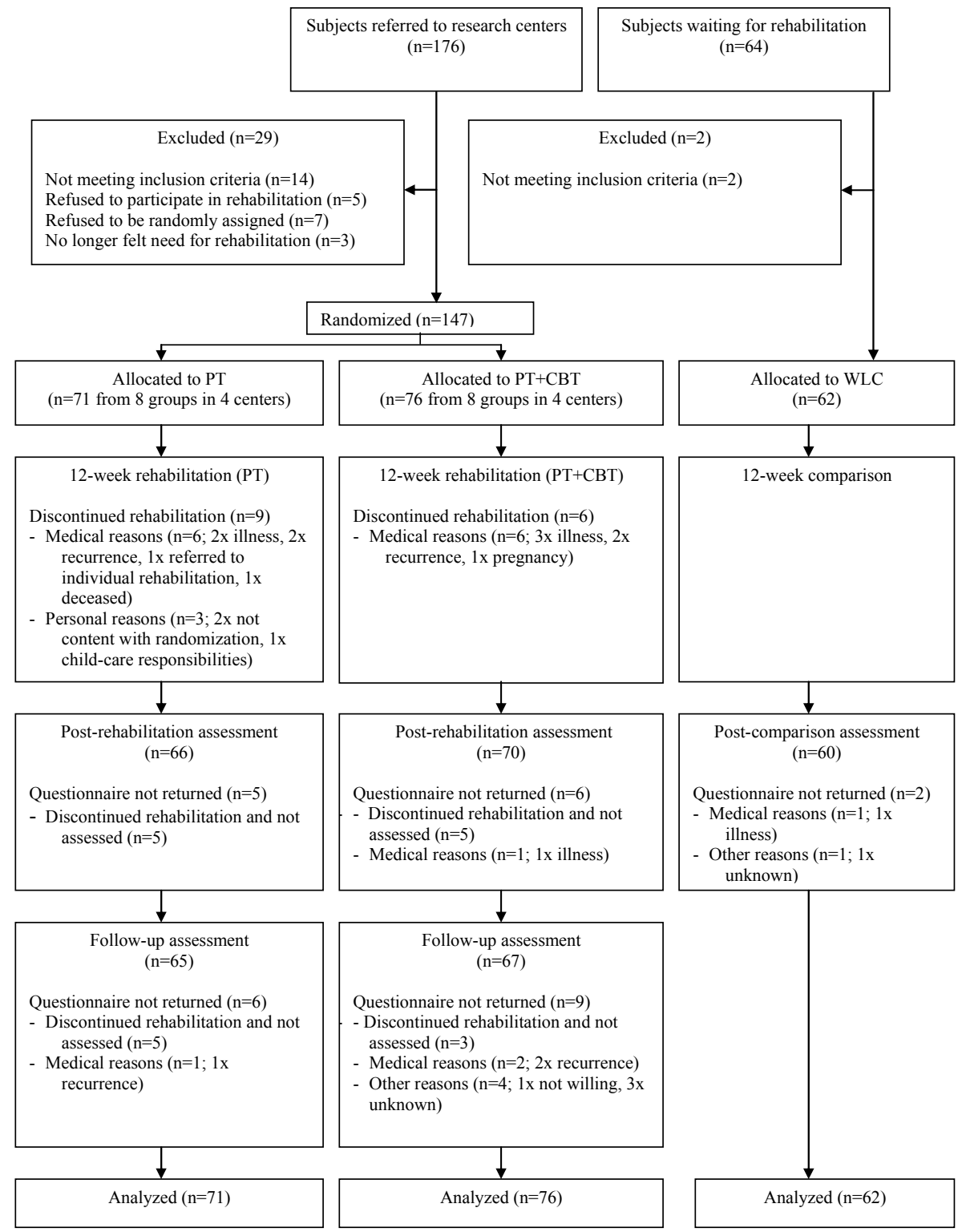

Figure 1. Flow of the participants through the study

Abbreviations: PT: physical training; PT+CBT: physical plus cognitive-behavioral training; WLC: waiting-list comparison. 


\section{The interventions}

The two interventions compared in this study were 1) a 12-week (twice weekly, 2-hour sessions) PT program, and 2) the same 12-week PT program plus a cognitive-behavioral training program (CBT) (weekly, 2-hour sessions). Both programs aimed at improving participants' role functioning and other aspects of QoL and supporting patients in coping with the physical and psychosocial consequences of cancer, with a primary focus on illness perceptions and self-management. The programs focused on patients' illness perceptions because coping strategies are guided by patients' perceptions of their illness and perceived identity (label and symptoms of illness), perceived causes, time-line (duration and course), and perceived consequences, controllability and curability (Leventhal and Carr 2001). Therefore, awareness or even adaptation of these perceptions might influence coping and support self-management. Self-management theory describes how patients cope with the consequences of their disease and their treatment by means of a circular process of goal selection, information collection, information processing and evaluation, decision-making, action and self-reaction (Mesters et al. 2002). Both programs were purposely developed as groupwise interventions to enhance fellow patient contacts.

The group format and specially included refreshment breaks in both PT and CBT provided opportunities for social comparison (Stanton et al. 1999), social support (Helgeson and Cohen 1996) and modeling (Bandura 1986). PT as well as CBT were tailor-made to individual participants through personalized exercises. PT was guided by two physiotherapists and CBT was guided by a psychologist, and a nurse, physiotherapist or social worker. All therapists were experienced in their profession and in the field of cancer rehabilitation. The experience of PT therapists in this field ranged from 2.5 to 6.3 years (median 5.1 years) and CBT therapists were working in cancer rehabilitation between 2.4 to 11.3 years (median 4.4 years). All therapists received group training to apply the standardized protocols: PT therapists for 1 day, CBT therapists for 2 days.

\section{Physical training}

PT included personalized physical exercise to improve exercise capacity, muscle strength, to reduce physical limitations and fatigue and to increase daily activity. Each session consisted of individual aerobic bicycle training (30 minutes), based on baseline graded exercise testing, muscle strength training (30 minutes), and group sports and games (60 minutes). Sports and 
games, such as badminton, soccer, swimming and balancing games, were aimed at promoting enjoyment in sports and improving self-efficacy in order to incorporate sporting activities into daily life and to adopt a physically active lifestyle. Additionally, patients received information on exercise physiology, illness perceptions and self-management to support them in regulating their physical training. Participants learned to use by themselves heart rate sport testers, the Borg Scale for dyspnea and fatigue (Borg 1982), and training $\operatorname{logs}$ to monitor and evaluate their performance, and they received feedback, information, and support from their therapists in regulating their performance. During the first 4 weeks participants followed a tailor-made basic training program, based on individual baseline testing. Then, in cooperation with the therapists, participants determined their personal goals for training from week 5 onward. They could choose one of four modules: improvement of physical condition, improvement of muscular strength, coping with fatigue, or handling limitations.

\section{Cognitive-behavioral training}

CBT included interactive psycho-education and structured self-management skills training. This training was formatted in line with a cognitivebehavioral problem-solving therapy protocol for individual cancer patients (Nezu et al. 1998) and a group problem-solving protocol successfully applied in patients with non-specific low back pain (Van den Hout et al. 2003; Kole-Snijders et al. 2006). CBT aimed at enabling participants to effectively solve their personal problems associated with cancer. To this end, they learned to apply self-management skills in striving for personal goals (e.g. in work, household, hobbies, physical activity, family relationships and social contacts). Generalization to daily life during and after rehabilitation was promoted by practicing activities during sessions and by homework assignments (maximally 30 minutes weekly). Every session was structured in: 1) recapitulation of the previous week's session and exchanging daily life experiences; 2) discussing home assignments; 3) introducing new topics or self-management skills; 4) practicing self-management skills; 5) introducing the next homework assignments; and 6) relaxation exercises. Participants used a workbook containing a summary of the training, self-management worksheets and assignments, as well as information on additional topics relevant to cancer patients. The first 3 weeks focused primarily on exchanging participants' experiences with cancer, psycho-education about stress, relaxation, fatigue, exercise physiology, illness perceptions, as well as on promoting optimism and self-efficacy for self-management. From week 4 
onward, participants were primarily trained in applying self-management skills to realize personal goals by practicing the following steps in the circular problem-solving process: 1) problem orientation; 2) problem definition and formulation, and goal setting; 3) generation of alternative solutions (brainstorming); 4) decision-making; and 5) solution implementation and verification.

\section{Measures}

Socio-demographic and medical variables were assessed using self-report questionnaires, with confirmation of medical data by the referring physicians.

General health-related QoL was measured using the RAND 36-item Health Survey (Van der Zee and Sanderman 1993; Ware and Sherbourne 1992). This multidimensional self-report questionnaire consists of four functional scales: role limitations due to physical problem (4 items), role limitations due to emotional problem (3 items), physical functioning (10 items), social functioning ( 2 items); three well-being scales: mental health (5 items), vitality (4 items) and pain ( 2 items), and a global scale: general health perception ( 5 items). The two role limitations subscales measure problems with work or other daily activities, either as a result of physical health problems, or due to emotional problems. One last item, health change, assesses the perceived change over the last year in general health status. After applying linear transformation according to the manual, scores of the scales ranged from $0-100$ with a higher score reflecting better health. The RAND-36 is a reliable and valid instrument widely used in patients with different somatic diseases. Cronbach's $\alpha$ of the subscales range from 0.71 to 0.92 (Van der Zee and Sanderman 1993; Van der Zee et al. 1996). The Cronbach's $\alpha$ in the present study ranged from 0.74 to 0.89 at baseline, from 0.75 to 0.87 at post test, and from 0.77 to 0.90 at 3 -month follow-up, indicating adequate-to-good internal consistency of all subscales. The Dutch RAND-36 manual provides reference scores of a random sample $(\mathrm{n}=1063$, $65 \%$ females, mean age 44.1 , range 18 - 89) of the general Dutch population (Table 3) (Van der Zee and Sanderman 1993).

Primary outcome in the comparison between both rehabilitation groups was role imitations (emotional), and the primary outcome in the comparison between both rehabilitation groups and WLC was role limitations (physical). 


\section{Statistical analysis}

A priori power analysis for a comparison between the randomized groups on the primary outcome role limitations (emotional) estimated a sample size of 64 participants in each group to detect a moderate effect-size $(d=0.50)$ with a power of 0.80 and a two-tailed alpha of 0.05 . Accounting for an estimated dropout of $10 \% 71$ participants in each group were needed.

Statistical analysis was performed using SPSS statistical software, version13.0, 2004 (SPSS 2004).

Longitudinal intention-to-treat analyses were conducted, using mixed linear regression models (Blackwell et al. 2006). In these analyses the program accounts for missing data based on the observed data. Firstly, the rehabilitation effect on QoL was tested in PT $+\mathrm{CBT}$ as compared with PT after rehabilitation and at 3-month follow-up. Level 1 was time; level 2 was participant. A factor "center" was also included because of the four-center study design. Because we used group randomization we considered to see group as level 3. However, after incorporating group, in most subscales the analyses failed, probably due to the small sample size (16 groups) at this higher level (Maas and Hox 2005) and small differences in QoL between groups, whereas in the subscales in which the analyses were successfully conducted, incorporating group level did not influence the results. We optimally adjusted for group effects by taking in account individual participants at level 2 as well as the factor center. Second, the same method of analysis was performed to test the rehabilitation effect on QoL in the rehabilitation groups as compared with WLC at post test, except that the factor "center" was not included, because WLC was not nested in the four centers. In these longitudinal analyses we corrected for any baseline differences between the rehabilitation groups and WLC.

ES were calculated according to Cohen as indices measuring the magnitude of a treatment effect. An ES $<0.2$ reflects "no effect," ES $\geq 0.2, \leq 0.5$ "small effect," $E S \geq 0.5, \leq 0.8$ "moderate effect" and $E S \geq 0.8$ reflects "large effect" (Cohen 1988).

Differences were explored in baseline socio-demographic and medical characteristics between included patients and those who met the inclusion criteria but refused participation or no longer felt a need for rehabilitation at baseline as well as between participants who stayed in the study and those who discontinued rehabilitation. In addition, differences were tested in socio-demographic and medical characteristics and in baseline QoL scores between the rehabilitation groups and WLC. Analysis of variance (ANOVA) 
was used for continuous data and Chi-Square tests were used for categorical data.

\section{Results}

No differences were found in baseline socio-demographic and medical characteristics between the 209 included patients (Table 1) and the 15 patients who met the inclusion criteria but refused participation or no longer felt a need for rehabilitation at baseline. Differences were found between the 194 participants who stayed in the study and the 15 participants who discontinued rehabilitation. Participants who stayed in the study were significantly more often working at baseline than were those who discontinued rehabilitation $(39.7 \%$ versus $13.3 \%)$ and they reported more often co-morbidity $(47.4 \%$ versus $20 \%)(\mathrm{p}<0.05)$. Participants with co-morbidity reported to receive medical treatment for one or more of the following problems: cardiac problems, vascular problems, diabetes, asthma, rheumatic problems, musculoskeletal problems, psychological problems or other complaints.

\section{Patient characteristics}

Table 1 shows participants' baseline characteristics. Groups were well balanced in almost all socio-demographic and medical variables. However, as compared to participants in both rehabilitation groups WLC participants were less educated, more often married and more of them had experienced a cancer recurrence $>3$ months before rehabilitation $(p<0.05)$. All participants, including those who had experienced a recurrence, had completed curative medical treatment at least 3 months before rehabilitation and had a minimum estimated life expectancy of 1 year. 
Table 1. Baseline characteristics ${ }^{a}$

\begin{tabular}{|c|c|c|c|c|c|}
\hline & PT & $\mathrm{PT}+\mathrm{CBT}$ & $\begin{array}{l}\text { Rehabilitation } \\
\text { groups }\end{array}$ & WLC & Overall \\
\hline & $n=71$ & $n=76$ & $n=147$ & $n=62$ & $n=209$ \\
\hline Age (years) & $49.9 \pm 11.3$ & $47.8 \pm 10.5$ & $48.8 \pm 10.9$ & $51.3 \pm 8.8$ & $49.5 \pm 10.4$ \\
\hline \multicolumn{6}{|l|}{ Sex } \\
\hline Female & $57(80.3)$ & $66(86.8)$ & $123(83.7)$ & $56(90.3)$ & $179(85.6)$ \\
\hline \multicolumn{6}{|l|}{ Educational level b } \\
\hline Low & $14(19.7)$ & $6(7.9)$ & $20(13.6)$ & $16(25.8)$ & $36(17.2)$ \\
\hline Middle & $32(45.1)$ & $40(52.6)$ & $72(49.0)$ & $32(51.6)$ & $104(49.8)$ \\
\hline High & $25(35.2)$ & $30(39.5)$ & $55(37.4)$ & $14(22.6)$ & $69(33.0)$ \\
\hline \multicolumn{6}{|l|}{ Marital status ${ }^{b}$} \\
\hline Married/living together & $51(71.8)$ & $53(69.7)$ & $104(70.7)$ & $55(88.7)$ & $159(76.1)$ \\
\hline \multicolumn{6}{|l|}{ Employment status } \\
\hline Employed at diagnosis & $53(74.6)$ & $54(71.1)$ & $107(72.8)$ & $46(74.2)$ & $153(73.2)$ \\
\hline Actually working at baseline & $23(32.4)$ & $32(42.1)$ & $55(37.4)$ & $24(38.7)$ & $79(37.8)$ \\
\hline \multicolumn{6}{|l|}{ Cancer type } \\
\hline Breast & $34(47.9)$ & $48(63.2)$ & $82(55.8)$ & $38(61.3)$ & $120(57.4)$ \\
\hline Hematological & $8(11.3)$ & $15(19.7)$ & $23(15.6)$ & $10(16.1)$ & $33(15.8)$ \\
\hline Gynecological & $11(15.5)$ & $6(7.9)$ & $17(11.6)$ & $7(11.3)$ & $24(11.5)$ \\
\hline Urologic & $6(8.5)$ & $3(3.9)$ & $9(6.1)$ & - & $9(4.3)$ \\
\hline Lung & $2(2.8)$ & $2(2.6)$ & $4(2.7)$ & $4(6.5)$ & $8(3.8)$ \\
\hline Colon & $2(2.8)$ & $1(1.3)$ & $3(2.0)$ & $2(3.2)$ & $5(2.4)$ \\
\hline Other & $8(11.3)$ & $1(1.3)$ & $9(6.1)$ & $1(1.6)$ & $10(4.8)$ \\
\hline \multicolumn{6}{|l|}{ Recurrence $>3$ months ago $b$} \\
\hline Yes & $7(9.9)$ & $7(9.2)$ & $14(9.5)$ & $15(24.2)$ & $29(13.9)$ \\
\hline No & $57(80.3)$ & $61(80.3)$ & $118(80.3)$ & $45(72.6)$ & $163(78.0)$ \\
\hline Unknown & $7(9.9)$ & $8(10.5)$ & $15(10.2)$ & $2(3.2)$ & $17(8.1)$ \\
\hline \multicolumn{6}{|l|}{ Metastases $>3$ months ago } \\
\hline Yes & $15(21.1)$ & $16(21.1)$ & $31(21.1)$ & $12(19.4)$ & $43(20.6)$ \\
\hline No & $55(77.5)$ & $59(77.6)$ & $114(77.6)$ & $49(79.0)$ & $163(78.0)$ \\
\hline Missing & $1(1.4)$ & $1(1.3)$ & $2(1.4)$ & $1(1.6)$ & $3(1.4)$ \\
\hline \multicolumn{6}{|l|}{ Treatment $>3$ months ago } \\
\hline Surgery & $62(87.3)$ & $64(84.2)$ & $126(85.7)$ & $51(82.3)$ & $177(84.7)$ \\
\hline Chemotherapy & $45(63.4)$ & $55(72.4)$ & $100(68.0)$ & $39(62.9)$ & $141(67.5)$ \\
\hline Radiotherapy & $41(57.7)$ & $43(56.6)$ & $84(57.1)$ & $41(66.1)$ & $123(58.9)$ \\
\hline Time since treatment (years) & $1.4 \pm 2.1$ & $1.2 \pm 1.3$ & $1.3 \pm 1.7$ & $1.9 \pm 2.7$ & $1.5 \pm 2.1$ \\
\hline \multicolumn{6}{|l|}{ Co-morbidity at start } \\
\hline Yes & $32(45.1)$ & $36(47.4)$ & $68(46.3)$ & $27(43.5)$ & $95(45.5)$ \\
\hline No & $39(54.9)$ & $40(52.6)$ & 79 (53.7) & $34(54.8)$ & $113(54.1)$ \\
\hline Missing & - & - & - & $1(1.6)$ & $1(0.5)$ \\
\hline
\end{tabular}




\begin{tabular}{|c|c|c|c|c|c|}
\hline & PT & $\mathrm{PT}+\mathrm{CBT}$ & $\begin{array}{l}\text { Rehabilitation } \\
\text { groups }\end{array}$ & WLC & Overall \\
\hline & $n=71$ & $n=76$ & $n=147$ & $n=62$ & $n=209$ \\
\hline \multicolumn{6}{|l|}{ Hormonal therapy at start } \\
\hline Yes & $20(28.2)$ & $31(40.8)$ & $51(34.7)$ & $19(30.6)$ & $70(33.5)$ \\
\hline No & $51(71.8)$ & $45(59.2)$ & $96(65.3)$ & $43(69.4)$ & $139(66.5)$ \\
\hline \multicolumn{6}{|l|}{ Other medication at start } \\
\hline Yes & $52(73.2)$ & $53(69.7)$ & $105(71.4)$ & $42(67.7)$ & $147(70.3)$ \\
\hline No & $19(26.8)$ & $20(26.3)$ & $39(26.5)$ & $18(29.0)$ & $57(27.3)$ \\
\hline Missing & - & $3(3.9)$ & $3(2.0)$ & $2(3.2)$ & $5(2.4)$ \\
\hline \multicolumn{6}{|l|}{ Contacts $<2$ months ago } \\
\hline Specialist & $2.2 \pm 2.1$ & $2.1 \pm 1.8$ & $2.2 \pm 1.9$ & $2.1 \pm 4.2$ & $2.1 \pm 2.8$ \\
\hline General practitioner & $1.7 \pm 1.9$ & $1.3 \pm 1.2$ & $1.5 \pm 1.6$ & $1.3 \pm 1.5$ & $1.4 \pm 1.6$ \\
\hline Physiotherapist & $2.2 \pm 4.3$ & $2.1 \pm 3.9$ & $2.2 \pm 4.1$ & $2.5 \pm 3.8$ & $2.2 \pm 4.0$ \\
\hline Psychologist & $0.3 \pm 0.7$ & $0.2 \pm 0.7$ & $0.3 \pm 0.7$ & $0.4 \pm 1.5$ & $0.3 \pm 1.0$ \\
\hline
\end{tabular}

\section{Attendance and adverse events}

Both rehabilitation groups completed $83.5 \%$ of 24 physical training sessions (PT + CBT $20 \pm 4.7$; PT 20 \pm 5.2 ) and PT + CBT completed $82.4 \%$ of 12 cognitive-behavioral sessions (9.9 \pm 2.4$)$. One participant in PT collapsed during rehabilitation and died at the first-aid station, which, after autopsy, the physician diagnosed as not related to the intervention. No further adverse events were reported.

\section{Quality of life}

At baseline no significant differences in QoL subscales were found between both rehabilitation groups and WLC. Participants in the study reported significantly lower QoL than did the general Dutch population (Table 3) in all domains $(\mathrm{p}<0.05)$, except for PT $+\mathrm{CBT}$ and WLC in health change and $\mathrm{PT}$ in pain.

Table 2 shows participants' QoL changes in PT + CBT as compared with PT after rehabilitation and at 3-month follow-up. The primary outcome was role limitations (emotional). PT $+\mathrm{CBT}$ showed no significant changes beyond those in PT, neither in role limitations (emotional) nor in the other domains of quality of life. 


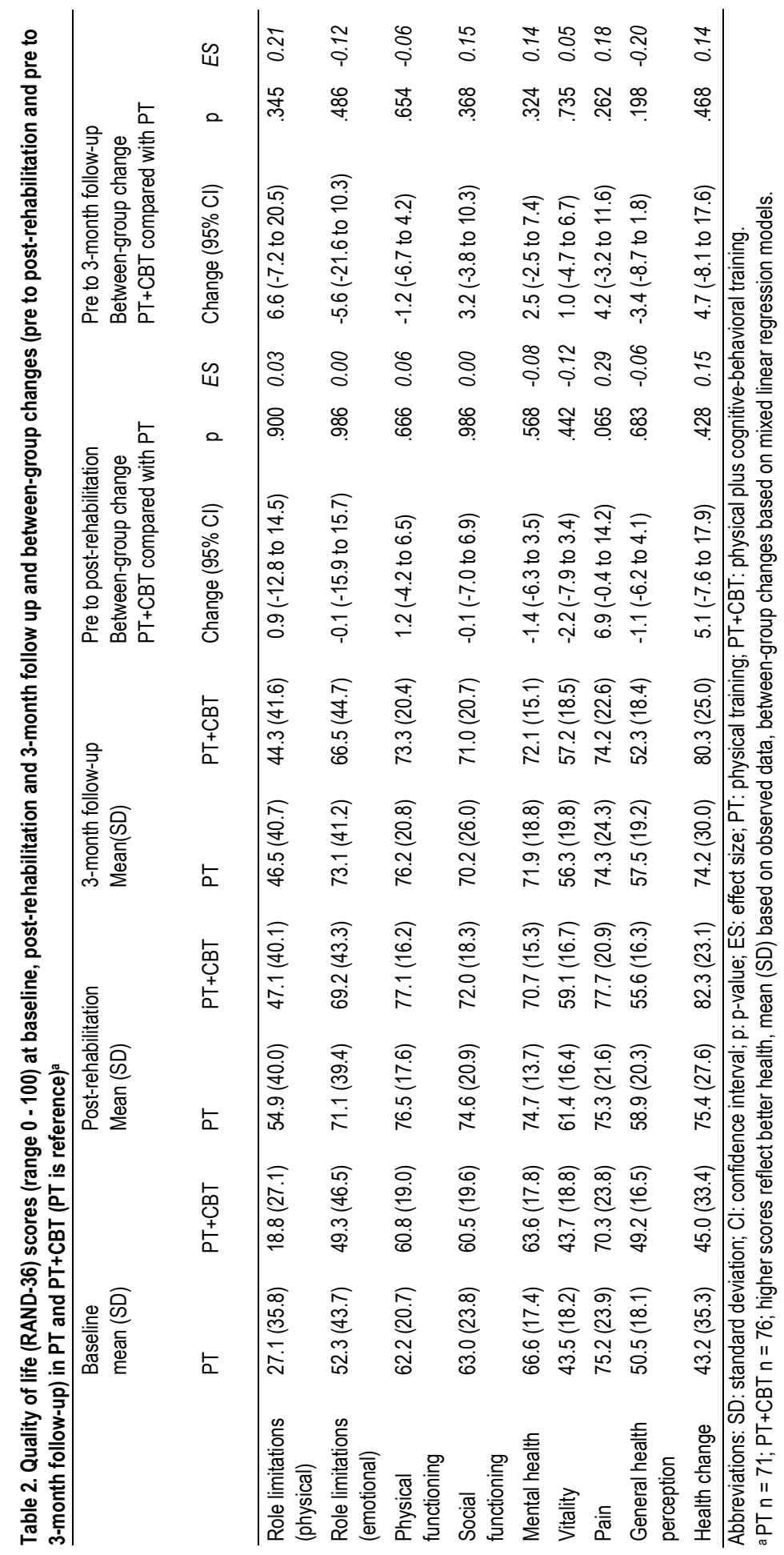


Table 3 shows participants' QoL changes in the rehabilitation groups as compared with the WLC between baseline and post test. The primary outcome was role limitations (physical). Compared with WLC, both rehabilitation groups showed significant improvements in role limitations (physical) (ES 0.66), representing a moderate treatment effect. Additionally, both rehabilitation groups showed significant improvements in physical functioning (ES 0.48), vitality (ES 0.54) and health change (ES 0.76), representing small-to-moderate treatment effects. Furthermore, all significant improvements were larger than a 5-point difference and a $10 \%$ change of scores, which are considered to be clinically relevant changes in the RAND-36 (Barrett et al. 2005; Walters and Brazier 2003). Lastly, within-group improvements in the rehabilitation groups were significant in all domains of QoL ( $\mathrm{p}<0.001)$, except in pain (data not shown). 
Table 3. Quality of life (RAND-36) scores (range 0 - 100) at baseline and post-rehabilitation and betweengroup changes (pre to post-rehabilitation) in the rehabilitation groups and WLC (WLC is reference) $)^{a<B} \mathrm{~B}^{\star}>$

$\begin{array}{lll}\text { Baseline } & \text { Post-rehabilitation } & \text { Pre to post } \\ \text { mean (SD) } & \text { Mean (SD) } & \text { Between-group change } \\ & & \text { Rehabilitation compared to WLC }\end{array}$

Rehabilitation WLC Rehabilitation WLC Change $(95 \% \mathrm{Cl}) \quad p \quad E S$ groups groups

\begin{tabular}{|c|c|c|c|c|c|c|c|}
\hline $\begin{array}{l}\text { Role limitations } \\
\text { (physical) }\end{array}$ & $22.8(31.7)$ & $25.0(31.7)$ & $50.9(40.1)$ & $32.9(39.7)$ & 20.8 (8.9 to 32.7$)$ & .001 & 0.66 \\
\hline $\begin{array}{l}\text { Role limitations } \\
\text { (emotional) }\end{array}$ & $50.7(45.1)$ & $59.0(42.7)$ & $70.1(41.3)$ & $66.7(41.6)$ & $12.2(-2.4$ to 26.8$)$ & .100 & 0.27 \\
\hline $\begin{array}{l}\text { Physical } \\
\text { functioning }\end{array}$ & $61.5(19.7)$ & $62.5(19.7)$ & $76.8(16.8)$ & $68.2(20.2)$ & 9.4 (5.1 to 13.6$)$ & .000 & 0.48 \\
\hline $\begin{array}{l}\text { Social } \\
\text { functioning }\end{array}$ & $61.7(21.7)$ & $64.7(19.8)$ & $73.3(19.6)$ & $71.6(20.1)$ & $4.5(-1.4$ to 10.4$)$ & .132 & 0.21 \\
\hline Mental health & $65.0(17.6)$ & $64.9(15.9)$ & $72.7(14.6)$ & $68.0(17.0)$ & $3.8(-0.2$ to 7.8$)$ & .063 & 0.22 \\
\hline Vitality & $43.6(18.5)$ & $45.3(16.9)$ & $60.2(16.5)$ & $51.7(18.7)$ & 9.8 (5.3 to 14.3$)$ & .000 & 0.54 \\
\hline Pain & $72.7(23.9)$ & $70.0(27.6)$ & $76.5(21.2)$ & $73.3(26.1)$ & $0.3(-5.8$ to 6.5$)$ & .916 & 0.01 \\
\hline $\begin{array}{l}\text { General health } \\
\text { perception }\end{array}$ & $49.8(17.2)$ & $53.6(21.5)$ & $57.2(18.3)$ & $56.3(19.4)$ & $3.6(-0.7$ to 8.0$)$ & .097 & 0.19 \\
\hline Health change & $44.1(34.2)$ & $45.2(32.6)$ & $78.9(25.5)$ & $55.4(32.6)$ & 25.7 (16.8 to 34.5$)$ & .000 & 0.76 \\
\hline \multicolumn{8}{|c|}{$\begin{array}{l}\text { Abbreviations: SD: standard deviation; Cl: confidence interval; } p \text { : p-value; ES: effect size; WLC: waiting-list } \\
\text { comparison. a Rehabilitation groups n=147; WLC n=62; higher scores reflect better health, mean (SD) based on } \\
\text { observed data, between-group changes based on mixed linear regression models. Reference scores Dutch general } \\
\text { population: mean (SD) (24): role limitations (physical) } 79.4 \text { (35.5); role limitation (emotional) } 84.1 \text { (32.3); physical } \\
\text { functioning } 81.9 \text { (23.2); social functioning } 86.9 \text { (20.5); mental health } 76.8 \text { (18.4); vitality } 67.4(19.9) ; \text { pain } 79.5(25.6) \text {; } \\
\text { general health perception } 72.7 \text { (22.7); health change } 52.4 \text { (19.4). }\end{array}$} \\
\hline
\end{tabular}

\section{Discussion}

Our hypothesis that multidisciplinary rehabilitation would demonstrate a greater improvement in role limitations (emotional) than would PT was not supported. Furthermore, multidisciplinary rehabilitation did not outperform PT in any other domains of QoL. Our hypothesis that both rehabilitation 
groups would outperform a WLC group was confirmed by a significant, clinically relevant improvement in role limitations (physical). Additionally, significant and clinically relevant improvements were found in physical functioning, vitality and health change (subjective health improvement). No improvements were found in general health perception, social functioning, role limitations (emotional), mental health, and pain. As participants did not report substantial pain at baseline, improvements in this domain were not likely to appear. The improvements in the rehabilitation groups, compared with WLC, were predominantly found in the physical domains. This might well be due to the PT component in both rehabilitation groups as PT may have most benefits in physical and functional domains, whereas psychosocial functioning is less likely to be considerably improved by PT (Courneya et al. 2003a; Courneya et al. 2003b; Karvinen et al. 2007; Rehse and Pukrop 2003).

Our randomized controlled study was the first study to compare combined cancer rehabilitation to PT and, at the same time, it was the first trial to include a 3-month follow-up of participants in comparing combined to single focused cancer rehabilitation. Our study showed that group-based cognitivebehavioral training had no effects on role limitation (emotional) or any other domains of QoL beyond those of group-based self-management PT after rehabilitation and at 3-month follow-up.

To date, one study, comparing combined rehabilitation (grouppsychotherapy plus home-based walking) to group-psychotherapy only, suggested the additive value of PT in two domains of QoL, functional wellbeing and fatigue (Courneya et al. 2003a). These findings, combined with our results, imply that PT should be included in cancer rehabilitation. Moreover, our study showed that up to 3-month follow-up combined cancer rehabilitation did not improve QoL beyond the effects of PT.

Although our findings suggest that $\mathrm{CBT}$ has no unique additive value for QoL above and beyond PT, such a conclusion would be premature. Cognitive-behavioral problem-solving interventions showed substantial beneficial effects on QoL in cancer patients in the longer term (Osborn et al 2006; Nezu et al. 2003). Moreover, positive long-term effects were reported of a group-based problem-solving intervention added to PT in non-specific low back pain patients (Van den Hout et al. 2003). Therefore, a long-term follow-up measurement in our two rehabilitation groups is needed.

It should be noted that our PT should not be regarded as being exercise only. First, the PT purposely applied a group-format that included opportunities for fellow patient contacts to enhance social comparison (Stanton et al. 
1999), social support (Helgeson and Cohen 1996) and modeling (Bandura 1986). Second, a self-management approach was applied to improve participants' self-efficacy in the PT. By including self-management in rehabilitation our study also distinguishes from earlier studies on group-based multidisciplinary cancer rehabilitation in patient populations similar to ours (Korstjens et al. 2006b; Van Weert et al. 2005) and from the group-based multidisciplinary program of Courneya et al. (2003a). A meta-analysis of randomized controlled studies of physical interventions reported an ES of 0.30 for QoL in cancer patients after medical treatment (Schmitz et al. 2005). The larger effects in our study (ES up to 0.76) in both rehabilitation groups as compared with no intervention might be explained by the group-format and self-management. At the same time, including these elements in PT may have prevented that CBT could improve participants' Qol beyond the benefits of PT.

A process analysis of both interventions showed high attendance in sessions and low dropout. This indicates that participants, who on average reported a low level of QoL at baseline, were highly motivated and that both rehabilitation programs were highly feasible in these cancer survivors. Hence, our findings can be generalized to cancer survivors experiencing physical and psychosocial problems that apply for rehabilitation on their own initiative.

Strengths of the present study were the randomized controlled design with intention-to-treat analyses, the 3-month follow-up, the supervised, standardized and theory-based intervention, large sample size, high attendance and low dropout rates.

A limitation of our study was that participants could not be randomly assigned to a WLC condition and that participants in the WLC group could not be assessed at 3-month follow-up. The reason for this was that cancer patients prefer to start rehabilitation as soon as possible after medical treatment. Nevertheless, as the comparison group participants were waiting for 12-week group-based multidisciplinary cancer rehabilitation programs in other Dutch centers using the same inclusion criteria, the comparison group was expected to be highly comparable to the rehabilitation groups. The groups showed to be well balanced in baseline QoL scores, and at the same time we corrected for any differences in socio-demographic and medical variables. Furthermore, we evaluated our tailor-made program at group level, but to further improve tailoring, additional research on predictors of successful treatment should show who profits most from what. Further research should focus on, for example, exercise capacity, muscle strength, anxiety and depression. 
To conclude, our study was the first to compare multidisciplinary cancer rehabilitation, combining physical and cognitive-behavioral training, to PT. Up to 3-month follow-up, multidisciplinary cancer rehabilitation did not outperform PT, neither in role limitation (emotional), nor in other domains of QoL. Compared with a WLC condition, the rehabilitation groups showed significant, clinically relevant improvements in role limitations (physical) and in several other domains of quality of life. 



\section{Chapter 5}

\section{Long-term effects on cancer survivors' quality of life of physical training versus physical training combined with cognitive- behavioral therapy: results from a randomized trial}

May, A.M., Korstjens, I., van Weert, E., Van den Borne, B., Hoekstra-Weebers, J.E.H.M., Van der Schans, C.P., Mesters, I., Passchier, J., Grobbee, D.E., \& Ros, W.J.G. (2008). Longterm effects on cancer survivors' quality of life of physical training versus physical training combined with cognitive-behavioral therapy: results from a randomized trial. Submitted. 


\section{Abstract}

Objective. To compare the effect of a group-based 12-week supervised physical training program with the effect of the same program combined with cognitive-behavioral therapy on cancer survivors' quality of life over a 1 -year period.

Design. Randomized trial with effect-measures at post-intervention and at 3- and at 9-month post-intervention.

Setting. Multicenter trial conducted at four Dutch centers, i.e. at one rehabilitation center and the rehabilitation units of two university medical centers and of one general hospital.

Participants. 147 cancer survivors entered the study (all cancer types, medical treatment $\geq 3$ months ago); 122 completed the 1-year follow-up.

Interventions. Supervised 12-week group-based physical training program (PT), i.e. aerobic and resistance exercise, and group sports, compared to PT combined with cognitive-behavioral therapy $(\mathrm{PT}+\mathrm{CBT})$.

Main outcome measures. European Organization for Research and Treatment of Cancer Quality of Life Questionnaire C30 (EORTC QLQ-C30) and the Physical Activity Scale for the Elderly (PASE).

Results. Multilevel linear mixed-effects models revealed no differential pattern in change of quality of life and physical activity between PT and $\mathrm{PT}+\mathrm{CBT}$. Effect estimates $(\mathrm{PT}+\mathrm{CBT}$ minus $\mathrm{PT})$ on the primary outcome global quality of life were -1.8 (95\% confidence interval -7.9 to 4.2$)$, -4.1 (10.2 to 2.0 ) and -0.6 (-6.8 to 5.7) at post-intervention, 3- and 9-month postintervention, respectively. In both $\mathrm{PT}$ and $\mathrm{PT}+\mathrm{CBT}$, quality of life and physical activity were significantly and clinically relevantly improved immediately following the intervention and also at 3- and at 9-month postintervention compared to pre-intervention $(\mathrm{p}<0.001)$.

Conclusion. Physical training has substantial and durable positive effects on cancer survivors' quality of life. Participants maintained physical activity levels once the program was completed. Combining physical training with cognitive-behavioral therapy does not add to these beneficial effects of physical training neither in the short-term nor in the long-term. Physical training should be implemented within the framework of standard care for cancer survivors. Physicians should encourage participation in exercise programs. 


\section{Introduction}

Cancer survivors often experience serious physical and psychosocial complaints caused by the disease and consequent treatment that may persist for many years (Bjordal et al. 1995). Cancer patients' quality of life declines most soon after diagnosis, attenuates with the time since diagnosis, but remains at a lower level in the long-term (Michael et al 2000; Sweeney at al. 2006). Recent reviews and meta-analyses showed that physical training has beneficial short-term effects on cancer survivors' quality of life (McNeely et al. 2006; Schmitz et al. 2005). However, evidence for the durability of these beneficial effects is lacking (McNeely et al. 2006). One randomized clinical trial examining the effects of a 12-week exercise program on quality of life in cancer survivors who completed primary cancer treatment showed promising results: quality of life improved immediately following the intervention and remained higher from post-intervention to 3-month followup (Milne et al. 2007). Daley et al. (2007) recently reported a beneficial effect on breast cancer survivors' quality of life directly following an 8-week physical training program compared to control, but differences between experimental and control disappeared at 4-month follow-up. To date, only one study investigating the effect of a 7-week group rehabilitation program on cancer survivors' quality of life included a one-year follow-up (Berglund et al 1994). However, this particular intervention consisting of physical training, information and training of coping skills did not lead to improvements in quality of life neither in the short-term nor in the long-term.

Quality of life is a multidimensional construct including physical as well as psychosocial dimensions (Aaronson et al. 1993). Studies on the effect of physical training in cancer survivors suggest that physical training is primarily associated with improved physical and functional aspects of quality of life, rather than the social and emotional domains (Courneya et al 2003a; Courneya et al. 2003b; Karvinen et al. 2007). A recent meta-analysis reported that cognitive-behavioral therapy had positive effects on mental health of cancer survivors (Osborn et al. 2006). Hence, combining physical training with cognitive-behavioral therapy might lead to greater improvements in quality of life by having benefits for both physical and psychosocial functioning. Therefore, we developed a rehabilitation program that integrated physical training and cognitive-behavioral therapy both based on principles of self-management (Creer 2000) to support cancer survivors in managing physical, psychological and social problems. We investigated the effects of that program on quality of life. 
We recently showed significant and clinically relevant improvements in cancer survivors' quality of life following physical training compared to waiting-list control (Korstjens et al. 2008a). Adding cognitive-behavioral therapy to our physical training program did not enhance the positive effects of physical training on quality of life and physical fitness at the short-term (Korstjens et al. 2008a; May et al. 2008). However, long-term effects may be different. Physical training may have early effects on quality of life, while cognitive-behavioral therapy, that at first confronts patients with personal worries and possibly increases distress (Helgeson et al. 2001; Pennebaker and Beall 1986), might have especially long-term benefits (Antoni et al. 2006; Nezu et al. 2003; Scheier et al. 2005). Hence, the long-term effects of physical training combined with cognitive-behavioral therapy on quality of life may be greater compared to the effects of physical training. The aim of the present study was to compare the long-term effects on quality of life of a 12-week self-management physical training intervention (PT), consisting of aerobic and resistance exercise, and group sports, with that of the same physical training intervention combined with cognitive-behavioral therapy $(\mathrm{PT}+\mathrm{CBT})$.

\section{Methods}

\section{Participants}

A prospective, randomized multicenter trial was conducted from February 2004 through January 2007. Four Dutch centers experienced in oncological rehabilitation participated in the trial, i.e. one rehabilitation center and the rehabilitation units of two university medical centers and of one general hospital. Patient inclusion criteria were: age $\geq 18$ years; last curative cancer treatment completed at least three months before study entry; estimated life expectancy of at least one year. Referral by a medical specialist or a general practitioner was needed who judged fulfillment of at least three of the following criteria to ascertain the need for rehabilitation: i.e. physical complaints, reduced physical capacity, psychological problems, increased levels of fatigue, sleep disturbances, and problems in coping with reduced physical and psychosocial functioning. Patients with cognitive disturbances, serious psychopathology, or emotional instability that might impede participation, or patients who were in need of intensive medical treatment, were 
excluded. The medical ethics committee of the University Medical Center Utrecht and the local research ethics committees approved the study.

\section{Recruitment and allocation}

Cancer survivors were informed about the study by various methods, including leaflets handed out by oncologists and general practitioners, information in the local newspapers and through the website (www.oncorev.nl). After written consent, eligible subjects were scheduled for baseline measurements and randomized to PT or PT + CBT. By design, $\mathrm{PT}$ and $\mathrm{PT}+\mathrm{CBT}$ were balanced in each center. Randomization at group level was applied; in each centre consecutive groups of eight to twelve eligible subjects were assigned to the randomly determined treatment to ascertain adequate numbers of participants in each group. Prior to enrolment of participants in the study, an independent researcher randomly determined the sequence of interventions at each center, using a randomization list. Until the first session, participants were blind to the rehabilitation group they were allocated to. Since PT + CBT and PT were open-label treatments, participants and therapists could not be blinded once the intervention started. Figure 1 shows the flow of participants through the trial. 


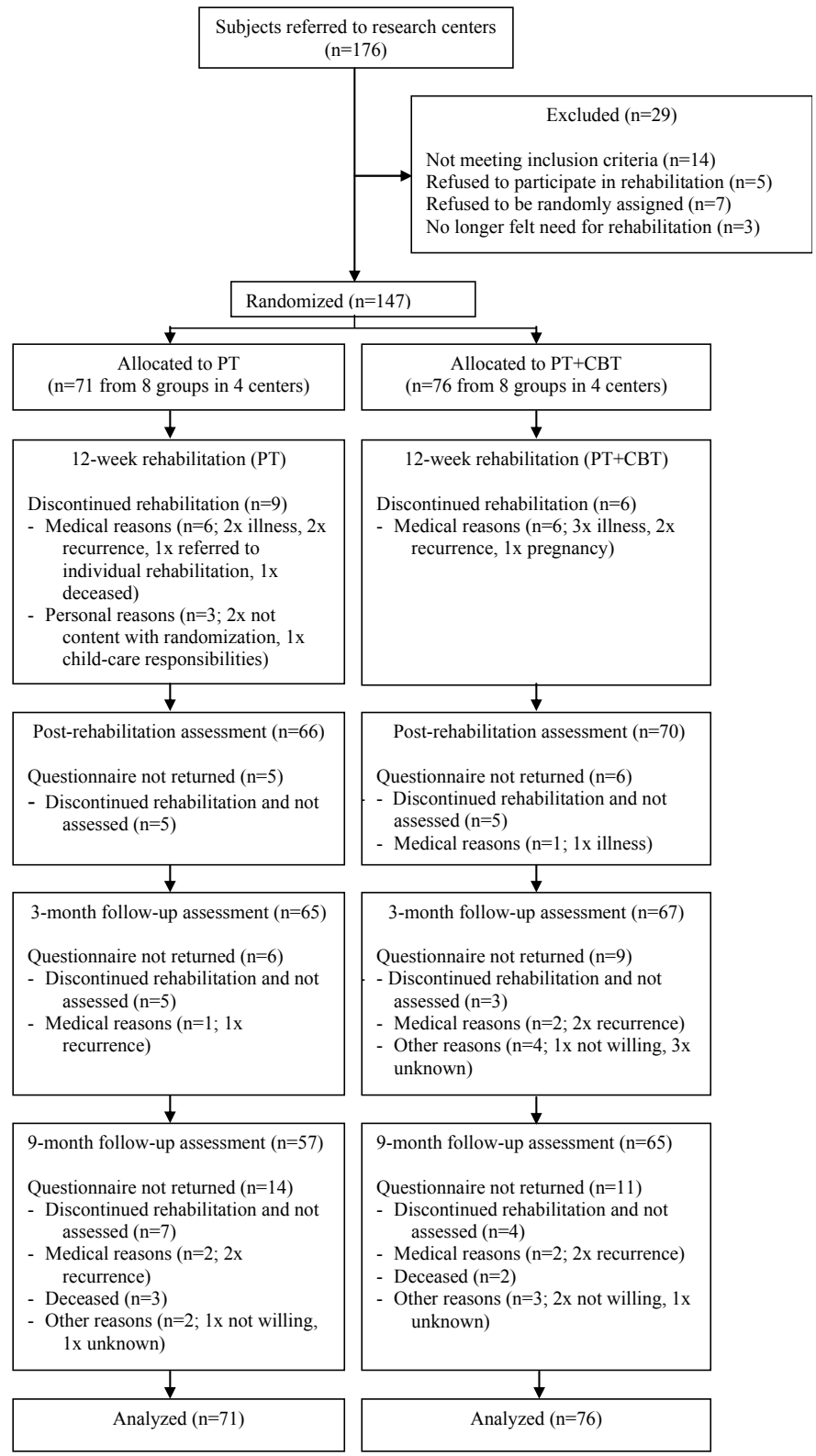

Figure 1. Flow of the participants through the study

Abbreviations: PT: physical training; PT+CBT: physical training plus cognitive-behavioral therapy. 


\section{Intervention}

The present intervention has been described in detail elsewhere (May et al. 2008; Van Weert et al. 2008).

Both components, PT and CBT, were based on principles of selfmanagement (Creer 2000): i.e. goal selection, information collection, information processing and evaluation, decision making, action and selfreaction. The intervention took place in groups of $8-12$ cancer survivors. PT was supervised by two physical therapists and CBT by a psychologist and a social worker. All therapists involved were trained to apply the standardized intervention protocol. They were experienced in their profession and in the field of cancer rehabilitation.

\section{Physical training}

Physical training (twice weekly, two hours per session) consisted of a personalized exercise program based on baseline exhaustive exercise testing. Each session consisted of individual exercise (aerobic bicycle training (30 minutes) and muscle strength training (30 minutes)) followed by group sports (60 minutes). During the training, the participants used heart rate monitors, the Borg Scale for dyspnoea and fatigue (Borg 1982) and training logs to monitor and evaluate their performance, and received feedback, information and support from their therapists in regulating their performance.

Aerobic bicycle training: Intensity was determined using the Karvonen formula (Karvonen and Vuorimaa 1988) that uses the peak heart rate $\left(\mathrm{HR}_{\text {peak }}\right)$ obtained from baseline exhaustive exercise testing and the heart rate at rest $\left(\mathrm{HR}_{\mathrm{rest}}\right)$ to calculate the training $\mathrm{HR}\left(\mathrm{HR}_{\mathrm{tr}}\right)$. Exercise training was performed at a $\mathrm{HR}_{\mathrm{tr}}$ of $\left(H R_{\text {rest }}+40 \%\right.$ to $50 \%$ of $\left.\left(H R_{\text {peak }}-H R_{\text {rest }}\right)\right)$ during the first four weeks and was gradually increased to $\left(H R_{\text {rest }}+70 \%\right.$ to $80 \%$ of $\left.\left(H R_{\text {peak }}-H R_{\text {rest }}\right)\right)$ in week 12 .

Muscle strength training: Resistance exercise of lower and upper extremities was based on the baseline 1-Repetition Maximum (1-RM). Training intensity started at $30 \%$ of the $1-\mathrm{RM}$ during the first week and was increased until 50 to $60 \%$ of 1-RM in week 12 .

Group sports: Sports, such as badminton, soccer, swimming and balancing games were performed with the aim being to promote enjoyment of sports and overcome any lack of confidence patients may have felt about exercising. 


\section{Cognitive-behavioral training}

Cognitive-behavioral training (once a week, two hours per session) was based on a cognitive-behavioral problem-solving protocol for individual cancer patients (Nezu et al. 1998) and a group problem-solving protocol (Van den Hout et al. 2003). During CBT, the participants learned to apply self-management skills (Creer 2000) in striving for personal goals (e.g. in physical activity, work, household, hobbies, family relations and social contacts). Generalization to daily life during and after rehabilitation was promoted by practicing activities during sessions and by homework assignments (maximally 30 minutes weekly). The first three weeks focused primarily on exchanging participants' experiences with cancer, psychoeducation about stress, relaxation, fatigue, exercise physiology, illness perceptions, as well as on promoting optimism and self-efficacy for selfmanagement. From week 4 onward participants were primarily trained in applying self-management skills to realize personal goals by practicing the following steps in the circular problem-solving process: 1) problem orientation; 2) problem definition and formulation, and goal setting; 3) generation of alternative solutions (brainstorming); 4) decision-making; and 5) solution implementation and verification.

\section{Outcomes}

Socio-demographic and medical data were collected using a self-report questionnaire. Medical data were confirmed by the referring physicians.

Quality of life was assessed by the multidimensional European Organization for Research and Treatment of Cancer Quality of Life Questionnaire C30 (EORTC QLQ-C30) (Aaronson et al. 1993), which assessed cancer-specific quality of life. The 30-item EORTC QLQ-C30 incorporates a global quality of life scale and five functional scales: namely, physical functioning, social functioning, role functioning, emotional functioning, cognitive functioning; and three symptom scales: fatigue, pain and nausea and vomiting. In this study we report the results of the global scale, the functional scales, and one symptom scale (i.e. fatigue) since these scales are most relevant for participants who have already completed primary treatment for cancer. After applying a linear transformation procedure according to the EORTC QLQC30 manual, the scores of the scales ranged from 0 to 100 . A higher score represents a higher quality of life at the global and functional scales, and a higher level of fatigue. The Cronbach's $\alpha$ in the present study were high for each of the seven scales $(0.7-0.9)$ at all measurement occasions. Differences 
of at least ten points were classified as a minimum clinically meaningful change. Changes of less than ten points were regarded as clinically irrelevant, and changes of more than 20 points were classed as large effects (Osoba et al. 1998).

The 12-item Physical Activity Scale for the Elderly (PASE), a valid and reliable questionnaire (Schuit et al. 1997) was used to assess whether the participants maintained being physical active once the program was completed. Questions deal with physical activities, such as leisure, sports, occupational, housework, and gardening. The questionnaire records the frequency of participation in these activities over the preceding seven days. Scoring procedures were derived from motion sensor counts, physical activity diaries and a global activity self-assessment. The total PASE-score is computed by multiplying the amount of time spent in each activity by the item weights and summing over all activities. The PASE generates a single composite score of physical activity that ranges from $0-400$. Mean PASE scores in a Dutch validation study in healthy elderly (age range 60-80) in the general population were: men: 97.9 (SD 26.8; $\mathrm{n}=10$ ) and women 71.9 (SD $45.9 ; \mathrm{n}=11$ ) (Schuit et al. 1997). A change of at least 20 points after a physical training program compared with no-intervention in patients after an ischemic stroke was considered as clinically relevant (Krarup et al. 2008).

\section{Data analysis}

For a comparison between the randomized groups on the primary outcome global quality of life a sample size of 64 participants in each group was estimated to detect a moderate effect-size $(\mathrm{d}=0.50)$ at post-intervention with a power of 0.80 and a two-tailed alpha of 0.05 . Accounting for an estimated dropout of $10 \% 71$ participants in each group were needed.

The effect of PT $+\mathrm{CBT}$ compared to PT on quality of life was tested at three, six and twelve months post-enrolment according to the intention-to-treat principle. Longitudinal analyses were conducted, using linear mixed-effects models while taking the different levels (training group, participant, and time) into account. The Akaike Information Criterion was used as a measure of fit of the models with a lower value indicating a better model fit. To determine whether changes of quality of life were maintained from postintervention to 9-month post-intervention statistical testing was performed whether the regression coefficients were different. In these analyses the program accounts for missing data based on the observed data. Statistical 
analysis was performed using $\mathrm{R}$ software, version 2.5.1. (www.rproject.org). Two-sided significance tests were used $(\alpha<0.05)$.

\section{Results}

Table 1 presents the baseline characteristics of the study participants. The groups were balanced on all socio-demographic and medical variables. Both rehabilitation groups completed $83.5 \%$ of 24 physical training sessions

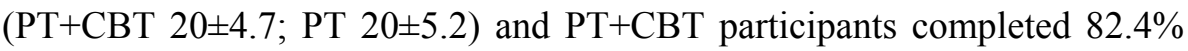
of 12 cognitive-behavioral sessions (9.9 \pm 2.4$)$. Of PT + CBT participants $92.1 \%, 88.2 \%$ and $85.5 \%$ completed the assessments at post-intervention and 3-month and 9-month post-intervention, respectively. Of PT participants 93.0\%, 91.5\% and $81.7 \%$ completed the assessments at post-intervention and 3-month and 9-month post-intervention, respectively (Figure 1). One participant, assigned to PT, collapsed during the intervention and deceased at the first-aid station. After autopsy, physicians judged this death to be unrelated to the intervention. No further adverse events were reported.

Table 2 shows the descriptive study measures over the 1-year study period. Multilevel linear mixed-effects models revealed no significant differences in changes over time in global quality of life between PT + CBT and PT (Figure 2). The physical, role, emotional, cognitive and social functioning subscales and the fatigue subscale all demonstrated the same pattern of change (Table $3)$. Significant improvements $(p<0.001)$ in global quality of life and in the other domains of quality of life were found in the PT group as well as in the $\mathrm{PT}+\mathrm{CBT}$ group immediately following the intervention, and at 3- and at 9month post-intervention compared to pre-intervention. Almost all of these improvements were larger than ten points, which is considered to be clinically relevant (Osoba et al. 1998). In both PT and PT + CBT, the short-term improvements in all domains of quality of life were maintained from postintervention to 9-month post-intervention except for a statistically significant but not clinically relevant decrease in global quality of life of PT participants ( $\mathrm{p}=0.04,3.9$-point difference).

Similar to the findings on quality of life, changes in physical activity were not different between PT $+\mathrm{CBT}$ and PT (Table 3). Significant improvements $(\mathrm{p}<0.05)$ in physical activity were found in the PT group as well as in the $\mathrm{PT}+\mathrm{CBT}$ group immediately following the intervention, and at 3- and at 9month post-intervention compared to pre-intervention, except for PT at 3- 
month follow-up. At 9-month post-intervention, physical activity levels were not different to post-intervention $(\mathrm{p}=0.9$ and $\mathrm{p}=0.8$ for PT $+\mathrm{CBT}$ and PT, respectively).

Table 1. Baseline characteristics*

\begin{tabular}{|c|c|c|c|}
\hline & $\begin{array}{l}\text { Overall } \\
(n=147)\end{array}$ & $\begin{array}{l}\mathrm{PT}+\mathrm{CBT} \\
(\mathrm{n}=76)\end{array}$ & $\begin{array}{l}\text { PT } \\
(n=71)\end{array}$ \\
\hline Age (years) & $48.8 \pm 10.9$ & $47.8 \pm 10.5$ & $49.9 \pm 11.3$ \\
\hline \multicolumn{4}{|l|}{ Sex } \\
\hline Female & $123(83.7)$ & $66(86.8)$ & $57(80.3)$ \\
\hline \multicolumn{4}{|l|}{ Educational level } \\
\hline Low & $20(13.6)$ & $6(7.9)$ & $14(19.7)$ \\
\hline Middle & $72(49.0)$ & $40(52.6)$ & $32(45.1)$ \\
\hline High & $55(36.8)$ & $30(39.5)$ & $25(35.2)$ \\
\hline \multicolumn{4}{|l|}{ Marital status } \\
\hline Married/living together & $104(70.7)$ & $53(69.7)$ & $51(71.8)$ \\
\hline Employed at diagnosis & $107(72.8)$ & 54 (71.1) & $53(74.6)$ \\
\hline Body Mass Index $\left(\mathrm{kg} \cdot \mathrm{m}^{2}\right)$ & $27.5 \pm 6.2$ & $27.4 \pm 6.7$ & $27.7 \pm 5.8$ \\
\hline \multicolumn{4}{|l|}{ Type of cancer } \\
\hline Breast & $82(55.8)$ & $48(63.2)$ & $34(47.9)$ \\
\hline Hematological & $23(16.6)$ & $15(19.7)$ & $8(11.3)$ \\
\hline Gynecological & $17(11.6)$ & $6(7.9)$ & $11(15.5)$ \\
\hline Urogenital & $9(5.5)$ & $3(3.9)$ & $6(8.5)$ \\
\hline Lung & $4(2.7)$ & $2(2.6)$ & $2(2.8)$ \\
\hline Colon & $3(2.0)$ & $1(1.3)$ & $2(2.8)$ \\
\hline Other & $9(6.2)$ & $1(1.3)$ & $8(11.3)$ \\
\hline \multicolumn{4}{|c|}{ Type of treatment (> 3 months ago) } \\
\hline Surgery & $126(85.7)$ & $64(84.2)$ & $62(87.3)$ \\
\hline Chemotherapy & $100(68.0)$ & $55(72.4)$ & $45(63.4)$ \\
\hline Radiotherapy & $84(57.1)$ & $43(56.6)$ & $41(57.7)$ \\
\hline Time post-treatment (years) & $1.3 \pm 1.7$ & $1.2 \pm 1.3$ & $1.4 \pm 2.1$ \\
\hline
\end{tabular}

Abbreviations: PT: physical training; PT+CBT: physical training plus cognitive-behavioral therapy. *Data presented as mean \pm standard deviation for continuous variables and frequency (percentage) for categorical variables. 
Table 2. Quality of life (EORTC QLQ-C30) and physical activity (PASE) over the 1-year study periodac'B">

\begin{tabular}{|c|c|c|c|c|}
\hline & $\begin{array}{l}\text { Baseline } \\
\text { Mean (SD) }\end{array}$ & $\begin{array}{l}\text { Post-intervention } \\
\text { Mean (SD) }\end{array}$ & $\begin{array}{l}\text { 3-month post- } \\
\text { intervention } \\
\text { Mean (SD) }\end{array}$ & $\begin{array}{l}\text { 9-month post- } \\
\text { intervention } \\
\text { Mean (SD) }\end{array}$ \\
\hline \multicolumn{5}{|c|}{ Global quality of life } \\
\hline PT & $57.7(16.7)$ & $74.1(14.8)$ & $71.1(18.7)$ & 70.2 (18.2) \\
\hline $\mathrm{PT}+\mathrm{CBT}$ & $56.5(18.4)$ & $71.3(16.4)$ & $66.5(20.7)$ & $67.9(19.6)$ \\
\hline \multicolumn{5}{|c|}{ Physical functioning } \\
\hline PT & $73.9(14.2)$ & $84.1(12.0)$ & $83.6(16.3)$ & $85.2(14.6)$ \\
\hline $\mathrm{PT}+\mathrm{CBT}$ & $71.7(13.3)$ & $85.5(10.5)$ & $83.8(13.3)$ & $82.5(15.6)$ \\
\hline \multicolumn{5}{|c|}{ Role functioning } \\
\hline PT & $59.6(24.2)$ & $74.0(24.3)$ & $74.9(23.4)$ & $77.0(24.5)$ \\
\hline $\mathrm{PT}+\mathrm{CBT}$ & $55.0(24.2)$ & $75.2(20.0)$ & $67.4(24.0)$ & $70.8(26.2)$ \\
\hline \multicolumn{5}{|c|}{ Emotional functioning } \\
\hline PT & $63.6(21.0)$ & $76.0(19.4)$ & $76.4(21.0)$ & $75.3(23.2)$ \\
\hline $\mathrm{PT}+\mathrm{CBT}$ & $60.7(22.1)$ & $75.8(18.9)$ & $73.6(19.2)$ & $70.6(22.5)$ \\
\hline \multicolumn{5}{|c|}{ Cognitive functioning } \\
\hline PT & $66.9(24.6)$ & $76.5(21.9)$ & $76.7(21.8)$ & $79.0(21.1)$ \\
\hline $\mathrm{PT}+\mathrm{CBT}$ & $62.3(27.0)$ & $72.4(21.0)$ & $72.9(24.2)$ & $73.3(22.6)$ \\
\hline \multicolumn{5}{|c|}{ Social functioning } \\
\hline PT & $64.5(26.4)$ & $83.8(20.5)$ & $81.8(24.4)$ & $82.8(23.3)$ \\
\hline $\mathrm{PT}+\mathrm{CBT}$ & $61.4(27.4)$ & $81.2(19.6)$ & $75.6(23.3)$ & $80.0(24.5)$ \\
\hline \multicolumn{5}{|l|}{ Fatigue } \\
\hline PT & $49.0(22.0)$ & $31.0(18.6)$ & $33.3(20.6)$ & $35.6(22.7)$ \\
\hline $\mathrm{PT}+\mathrm{CBT}$ & $52.8(24.5)$ & $34.8(17.5)$ & $37.5(24.1)$ & $36.2(25.3)$ \\
\hline \multicolumn{5}{|c|}{ Physical activity } \\
\hline PT & $110.0(57.4)$ & $136.8(78.1)$ & $118.0(72.3)$ & $140.4(87.1)$ \\
\hline $\mathrm{PT}+\mathrm{CBT}$ & $116.1(60.5)$ & $140.5(77.5)$ & $140.3(81.3)$ & $138.9(80.9)$ \\
\hline
\end{tabular}

Abbreviations: EORTC QLQ-C30: European Organization for Research and Treatment of Cancer Quality of Life Questionnaire C30 (range 0 - 100); PASE: Physical Activity Scale for the Elderly (range 0 - 400); SD: standard deviation; PT: physical training; PT+CBT: physical training plus cognitive-behavioral training. a PT: $n=71$ at baseline, $n=66$ at post-intervention, $n=65$ at 3-month post-intervention and $n=58$ at 9-month post-intervention; PT+CBT : $n$ $=76$ at baseline, $n=70$ at post-intervention, $n=67$ at 3-month post-intervention and $n=65$ at 9-month postintervention. "The intervention period comprised twelve weeks. 


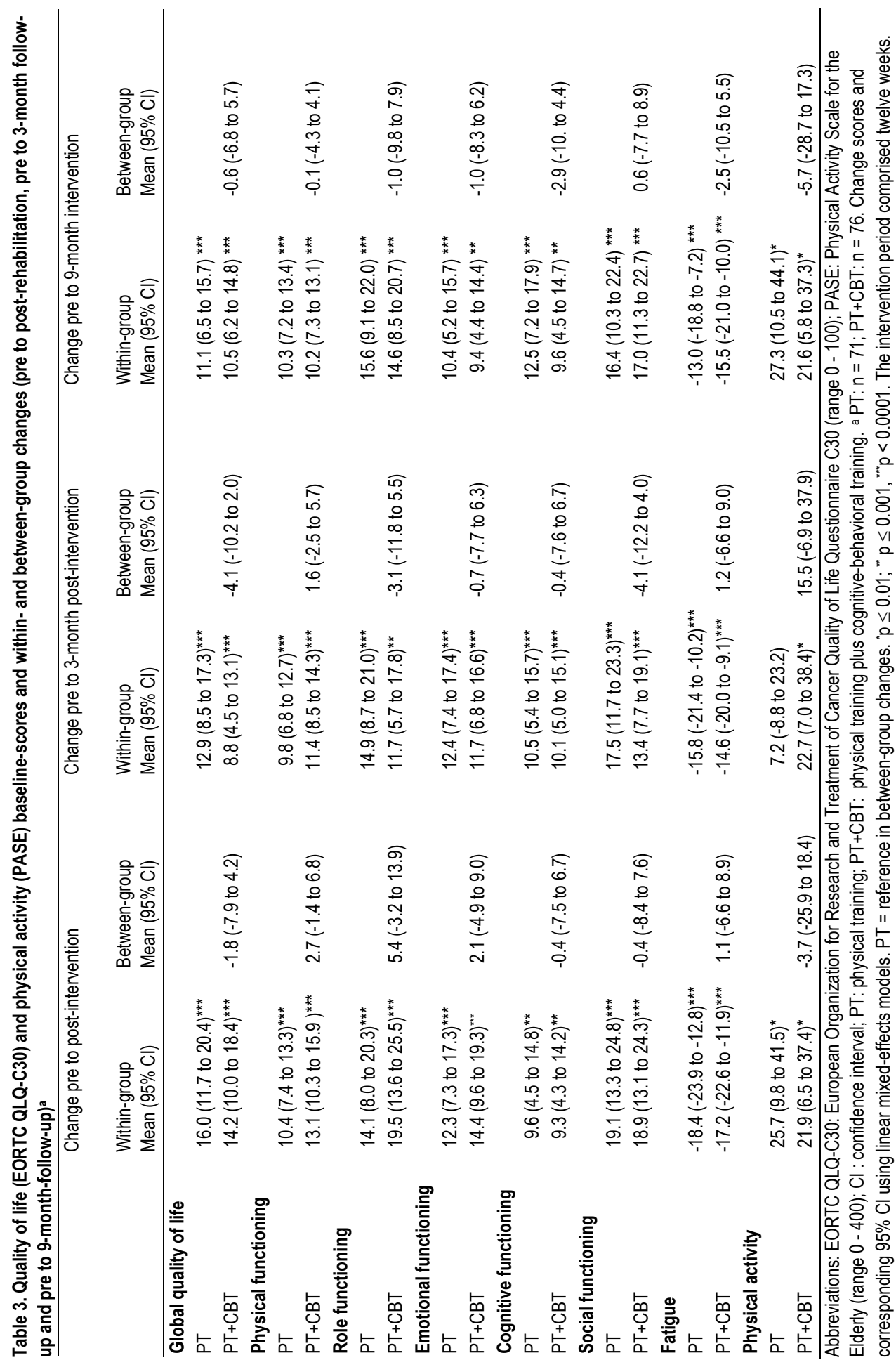



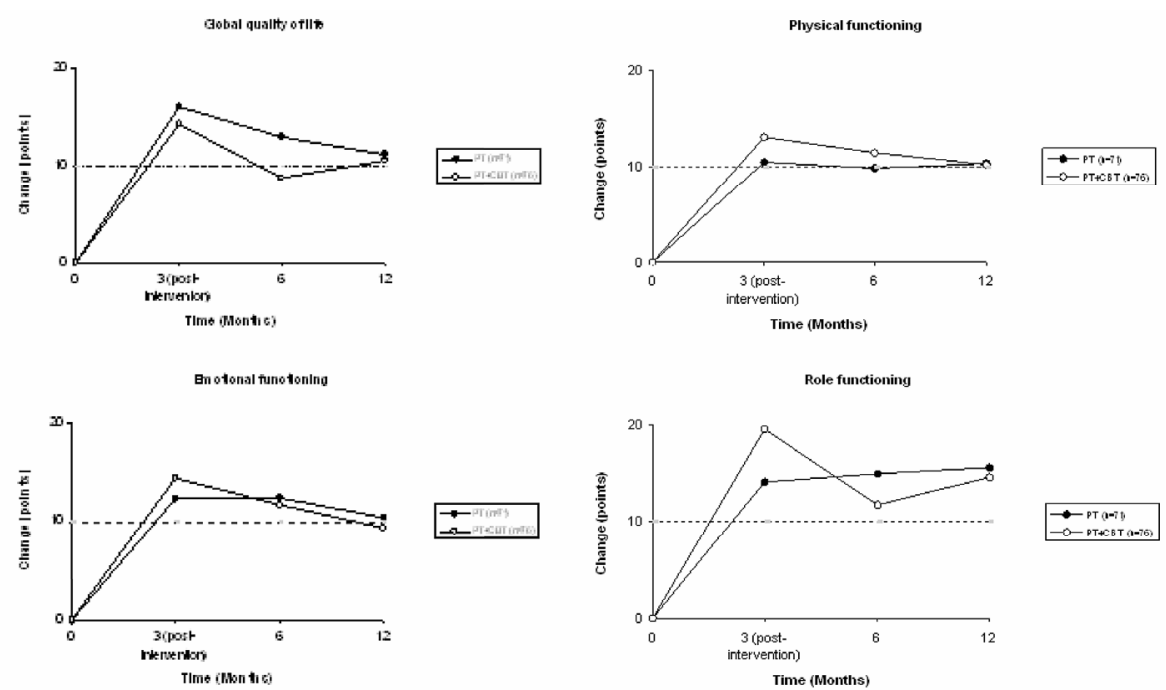

Figure 2. Change of quality of life measured using the EORTC QLQ-C30 from baseline to twelve months by intervention assignment

Abbreviations: PT: physical training; PT+CBT: physical plus cognitive-behavioral therapy EORTC QLQ-C30:

European Organization for Research and Treatment of Cancer Quality of Life Questionnaire C30. The dashed line represents the minimally clinically-important difference.

\section{Discussion}

The results of this randomized trial show that combining a self-management physical training program for cancer survivors with structured cognitivebehavioral therapy does not add to the beneficial effects of physical training neither in the short-term nor in the long-term.

Quality of life was improved following 12-week self-management physical training consisting of aerobic training, resistance exercise, and group sports in cancer survivors. Nine months after the intervention, improvements in global quality of life, physical, role, emotional, cognitive and social functioning and fatigue were maintained and were clinically relevant. Only participants in the PT group reported a decrease in global quality of life from post-intervention to 9-month post-intervention. However, this small decrease was not clinically relevant and the improvement in global quality of life at 9month post-intervention was still clinically relevant compared to preintervention. Global quality of life at 9-month post-intervention of PT participants was comparable to that of $\mathrm{PT}+\mathrm{CBT}$ participants. Moreover, the 
participants maintained their physical activity levels once the program was completed.

Participants in the trial were well motivated to participate in the intervention and applied for participation on their own initiative. Also, their attendance rates remained high during the intervention. Their quality of life appeared to be low at baseline. The results can be generalized to cancer survivors with physical and/or psychosocial problems who voluntarily apply for a physical training program.

To appreciate the findings some aspects of the study need to be addressed. Strengths of the present study were the length of follow-up, the use of intention-to treat analyses, the supervised, standardized intervention, the large sample size, high attendance rates and low drop-out rates. A limitation of the study was the lack of a control group for the long-term effects. We included a waiting-list comparison group for the evaluation of the effects immediately after our intervention, and showed significant improvements in quality of life in the intervention condition compared to control (Korstjens et al. 2008a; Korstjens et al. 2006a). However, for feasibility reasons, due to the facts that cancer survivors were highly motivated to attend an exercise intervention (Stull et al. 2007) and that alternatives were available in the Netherlands, a control group could not be assessed at 3-month and 9-month post-intervention. Therefore, we cannot definitely conclude that the maintained improvements in quality of life are specific to our intervention. Nonetheless, considering that quality of life was significantly and clinically relevantly improved directly following the intervention compared to waitinglist control patients and that these improvements were maintained at 9-month post-intervention, some evidence is provided that the beneficial long-term effects are not simply attributable to the passage of time and may likely be a consequence of the intervention.

The changes of quality of life in PT $+\mathrm{CBT}$ and PT were of a similar magnitude. It is unlikely that including more subjects in the study would have changed the conclusion that cognitive-behavioral therapy did not add to the beneficial effects of physical training. A post-hoc power analysis using the change scores found in the present study revealed that more than 1100 participants per group would have been required to show statistically significant differences in improvement of quality of life between PT + CBT and PT. A study of this size would not be feasible, and it is also doubtful whether a significant difference here would have sufficient clinical relevance. The fact that adding cognitive-behavioral therapy did not have additional effects does not allow the conclusion that cognitive-behavioral therapy by itself may not 
improve quality of life in cancer survivors as we did not include a group who received cognitive-behavioral therapy alone in our design.

Three other studies (Berglund et al. 1994; Daley et al. 2007; Milne et al. 2007) reported long-term results following an exercise intervention for cancer patients who had completed their cancer-related treatment. Similar to our findings in a mixed group of cancer survivors, Milne et al. (2007) showed that quality of life of breast cancer survivors was improved directly after and three months following a 12-week combined aerobic and resistance exercise intervention. Contrary to the findings in the present trial, they reported that quality of life still increased from post-intervention to 3-month post-intervention. This effect may be partly due to the attention given to the participants during regularly telephone calls (every three weeks) during follow-up, whereas we did not approach our participants during follow-up. Daley et al. (2007) showed a beneficial effect on breast cancer survivors' quality of life directly following an 8-week physical training program when compared to control that was not maintained at 4-month follow-up. To date, only one study included a 1-year follow-up (Berglund et al. 1994). This 7week group rehabilitation program, however, did not lead to improvements in quality of life on the short-term and the long-term. This could well reflect the short duration of the intervention and the low intensity of the program, which included only four information sessions, three coping skills training sessions and four light-intensity physical training sessions.

Our findings are in contrast to previous reports suggesting that the effect of cognitive-behavioral therapy may become more prominent over longer follow-up times (Antoni et al. 2006; Nezu et al. 2003). An explanation may be that the physical training intervention in the present trial comprised a selfmanagement approach. It has been shown that including social cognitive components, such as self-management, in an intervention may have positive effects on quality of life in cancer patients (Graves 2003). Furthermore, PT was offered in a group-format that provides opportunities for social interaction, social comparison and group support that might improve self-efficacy and subsequently quality of life (Graves 2003).

Despite the fact that primary treatment has been successfully completed, cancer survivors often report long-lasting physical and psychological complaints (Michael et al. 2000; Bower et al. 2006; Elbl et al. 2006). The present physical training program was developed based on the best available evidence (Van Weert et al. 2008). However, research on the optimal content and extent of a physical training program for cancer survivors is scarce. Future research should focus on whether an exercise program should consist 
of aerobic exercise, resistance exercise or a combination of both. Furthermore, the optimal duration of an intervention, the number of training sessions needed per week to accrue health benefits, and application of booster sessions post-intervention need to be investigated. Moreover, research is needed to reveal the characteristics of cancer survivors who benefit most from exercise programs.

In conclusion, the short- and long-term quality of life of cancer survivors may improve from participation in physical training programs. Adding cognitive-behavioral therapy to supervised group-based self-management physical training appears not to further enhance the beneficial effects of physical training alone. Physical training should be considered as a useful component in the standard care for cancer survivors. Physicians should encourage participation in exercise programs. 



\section{Chapter 6}

\section{Effects of cancer rehabilitation on problem- solving and distress: a RCT comparing physical and cognitive-behavioral training versus physical training}

Korstjens, I., Mesters, I., May, A. M., Van Weert, E., Van den Hout, J.H.C., Ros, W. J. G., Hoekstra-Weebers, J. E. H. M., Van der Schans, C.P., \& Van den Borne, B. Effects of cancer rehabilitation on problem-solving and distress: a RCT comparing physical and cognitivebehavioral training versus physical training. To be submitted as a shorter version of chapter 6 . 


\section{Abstract}

Objective. We compared the effects on problem-solving and distress of a 12-week group-based self-management cancer rehabilitation program, combining comprehensive physical training (PT, twice weekly) and cognitive-behavioral training (CBT, once weekly), with the effect of comprehensive physical training only $(\mathrm{PT})$. We expected that $\mathrm{PT}+\mathrm{CBT}$ would outperform PT in improvements in problem-solving, as well as distress (anxiety and depression), and that higher distressed participants would benefit most from adding CBT to PT.

Method. Cancer survivors (48.8 110.9 years of age), all cancer types, medical treatment completed $\geq 3$ months ago) were randomly assigned to PT + CBT $(n=76)$ or PT $(n=71)$. Problem-solving and distress (anxiety and depression) were measured using the SPSI-R and the HADS, respectively, before and after rehabilitation, and at three and nine months after rehabilitation.

Results. Longitudinal intention-to-treat analyses showed no differential pattern in change between PT + CBT and PT. Participants in both conditions showed significant improvements in one domain of problem-solving (negative problem orientation), as well as in distress (anxiety and depression) after rehabilitation and these improvements were maintained at three and nine months after rehabilitation. Higher distressed participants did not profit more from adding CBT to PT than participants with lower distress.

Reduced negative problem orientation was associated with reduced anxiety and depression.

Conclusion. Comprehensive physical training had significant and durable positive effects on cancer survivors' negative problem orientation, as well as on anxiety and depression. Adding cognitive-behavioral training did not add to these beneficial effects, neither in the short-term nor in the longterm. 


\section{Introduction}

After cancer diagnosis and its treatment about $26 \%$ of cancer survivors express a need for professional support in managing physical, psychological and social problems (Van Harten et al. 1998). To address such difficulties, problem-solving theory might provide some guidance.

In short, problem-solving is a general coping strategy, by which people attempt to find effective or adaptive solutions to stressful problems (Chang et al. 2004; D'Zurilla and Nezu 2007; Nezu 2004; Nezu et al. 1998; Nezu et al. 1999a). Their coping efforts are aimed at changing the problematic nature of a situation itself, i.e. problem-focused coping, changing their reactions to such situations, i.e. emotion-focused coping, or both (Lazarus 1993). Problem-solving involves two processes. Firstly, problem orientation, positive or negative, is mainly a motivational process involving a person's generalized thoughts and feelings about problems and his own coping ability. Secondly, problem-solving style, i.e. rational problem-solving, impulsive/carelessness style and avoidance style, refers to people's cognitive-behavioral activities aimed at finding solutions to particular problems. The circular problemsolving process contains five steps: problem orientation, followed by systematically applying four rational problem-solving skills, i.e. problem definition and formulation (including goal-setting), generation of alternative solutions, decision making, and solution implementation and verification. Problem-solving training aims to improve a person's problem-solving, i.e. to enhance one's positive problem orientation, decrease one's negative problem orientation, increase one's rational problem-solving, and decrease one's impulsive/carelessness and avoidance styles (D'Zurilla and Nezu 2007; Nezu 2004). So far research has confirmed that in cancer patients particularly more negative problem orientation was correlated with more anxiety and depression (Nezu et al. 1999b). Moreover, more negative problem orientation and less rational problem-solving predicted more anxiety and depression in cancer survivors years after medical treatment (Nezu et al. 1999b). Hence, a meta-analysis showed that cognitive-behavioral problemsolving training may decrease cancer survivors' distress (Osborn et al. 2006). Specifically, 10-week problem-solving training for highly distressed cancer patients showed large improvements in problem-solving and distress that were maintained at one-year follow-up (Nezu et al. 2003; Osborn et al. 2006). Meta-analyses of physical interventions in cancer survivors reported, besides benefits for physical functioning, also modest positive effects on distress in the short-term (Conn et al. 2006; McNeely et al. 2006; Schmitz et 
al. 2005). Therefore, adding problem-solving training to physical training might address cancer survivors' physical, psychological and social problems optimally and might have especially additional effects on distress (anxiety and depression) in the long-term. Furthermore, the additional value of problem-solving training might be more prominent in cancer survivors with higher pre-rehabilitation distress than in cancer survivors experiencing less distress.

We developed a 12-week group-based self-management rehabilitation program $(\mathrm{PT}+\mathrm{CBT})$ combining a comprehensive physical training $(\mathrm{PT})$ (integrating self-management techniques in 2-hour exercise and sports sessions, twice weekly) and a cognitive-behavioral problem-solving training (CBT) (2-hour sessions, once weekly). In recent studies we compared PT and $\mathrm{PT}+\mathrm{CBT}$ and showed that cancer survivors' physical fitness (May et al. 2008) and quality of life (Korstjens et al. 2008a; May et al. 2007b) equally improved with both interventions. For this paper, we hypothesized, firstly, that compared to the effects of PT, PT $+\mathrm{CBT}$ would show more short-term and long-term positive effects on problem-solving, anxiety and depression. Primary outcome was anxiety as prevalence of anxiety in cancer populations is higher than that for depression (Sheard and Maguire 1999) and because meta-analyses reported that psychological interventions in these populations show larger beneficial effects on anxiety than on depression (Sheard and Maguire 1999; Osborn et al. 2006). Secondly, we hypothesized that initially higher distressed participants would profit more from adding CBT to PT than participants with lower pre-rehabilitation distress, and thirdly, that improvements in problem-solving would be associated with improvements in anxiety and depression.

\section{Methods}

\section{Study design}

In this four-center prospective controlled study, participants were randomly assigned to $\mathrm{PT}$ or $\mathrm{PT}+\mathrm{CBT}$. Measurements were performed before and after rehabilitation (12 weeks after baseline), at 3-month follow-up (6 months after baseline) and at 9-month follow-up (one year after baseline). 


\section{Settings and participants}

The Dutch centers involved were: two university medical centers, one general hospital and one rehabilitation center. The medical ethics committee of the University Medical Center Utrecht and the local research ethics committees approved the study. Written informed consent was obtained from each participant. Eligible for the study were cancer patients (age $\geq 18$ years) who successfully completed curative cancer treatment, i.e. minimum estimated life expectancy of one year, at least three months ago. Referral was required by a medical specialist or a general practitioner who checked whether at least three of the following criteria were met: having physical complaints, reduced physical capacity, psychological problems, increased fatigue, sleep disturbances, and problems in coping with reduced physical and psychosocial functioning due to cancer. Patients were excluded in case of restrictive side effects from medication, serious cognitive disturbances, psychopathology, or emotional instability that might impede participation in rehabilitation, or if they needed intensive medical treatment or rehabilitation.

\section{Recruitment and allocation}

Leaflets handed out by general practitioners or by medical professionals in hospitals, local newspapers, radio and television, and a website (www.oncorev.nl) informed cancer patients about the program and the study. Ideally, patients in need for rehabilitation are referred by their doctors. In practice, many patients apply for rehabilitation on their own initiative, and then, are requested to obtain their doctor's referral to check the selection criteria (Korstjens et al. 2008b). Patients who contacted a center involved in the study received a program and study information package, containing an information letter, an informed consent form, a screening questionnaire and referral papers for their medical specialists or general practitioners. Each center delivered one group at a time. In each center consecutive groups of 8 12 eligible subjects were assigned to PT or PT $+\mathrm{CBT}$ and were scheduled for baseline measurements. Randomization at group level was applied; before enrolling participants in the study, the sequence of $\mathrm{PT}$ and $\mathrm{PT}+\mathrm{CBT}$ groups at each center was determined by an independent researcher from the University Medical Center Utrecht using a randomization list. After randomization, the sequence of treatments in each center was fixed and could not be influenced by research centers or researchers. Until the first session, participants were blinded to the rehabilitation group they were allocated to. Center $1(\mathrm{n}=15)$ delivered one PT and one PT+CBT group, centers $2(\mathrm{n}=$ 
44) and $3(n=39)$ each delivered two PT and two PT+CBT groups, and center $4(n=49)$ delivered three PT and three PT+CBT groups. Recruitment and assessments of participants occurred between February 2004 and January 2007 (Figure 1).

\section{The interventions}

The two interventions compared in this study were 1) a 12-week (twice weekly, 2-hour sessions) comprehensive group-based self-management physical training program (PT), and 2) the same comprehensive physical training program plus a cognitive-behavioral training program (CBT) (weekly, 2-hour sessions). Both programs aimed at improving participants' quality of life. PT and CBT were based on principles of self-management: patients cope with the physical and psychosocial consequences of their disease and their treatment by means of a circular process of goal selection, information collection, information processing and evaluation, decisionmaking, action and self-reaction (Mesters et al. 2002). Both programs were purposely developed as group-wise interventions to enhance fellow patient contacts. The group format in both PT and CBT provided opportunities for social comparison (Stanton et al. 1999), social support (Helgeson and Cohen 1996) and modeling (Bandura 1986). Additionally, both PT and CBT were tailor-made by fitting the activities to individual participants. PT was guided by two physiotherapists and CBT was guided by a psychologist, and a nurse, physiotherapist or social worker. All therapists were experienced in their profession and in the field of cancer rehabilitation. The experience of PT therapists in this field ranged from 2.5 to 6.3 years (median 5.1 years) and CBT therapists were working in cancer rehabilitation between 2.4 to 11.3 years (median 4.4 years). All therapists received group training to apply the standardized self-management protocols: PT therapists for one day, CBT therapists for two days.

\section{Comprehensive physical training}

PT, described by Van Weert and colleagues (2008), integrated selfmanagement principles and focused on physical activity. In accordance with self-management-principles, participants learned to use heart rate monitors, the Borg Scale for dyspnoea and fatigue (Borg 1982) and training logs to monitor and evaluate their performance, and they received feedback, information and support from their therapists in regulating their performance. In short, each session consisted of individual aerobic bicycle training (30 minutes), muscle strength training (30 minutes), and group sports and games 
(60 minutes). Sports and games, such as badminton, soccer, and swimming, were aimed at promoting enjoying sports and improving self-efficacy in order to stimulate participants to incorporate sporting activities into daily life and to adopt a physically active lifestyle. Additionally, patients received information on exercise physiology, illness perceptions and selfmanagement to support them in regulating their physical training. During the first four weeks participants followed a tailor-made basic training program, in which training intensity was based on individual exhaustive exercise testing at baseline. In cooperation with the therapists, participants determined their personal goals for training from week 5 onward. Then, training intensity also depended on personal goals, i.e. improvement of physical condition, improvement of muscular strength, coping with fatigue, and/or handling limitations.

\section{Cognitive-behavioral training}

CBT focused on training self-management skills to enable participants to effectively solve their personal problems associated with cancer. This training was formatted in line with a cognitive-behavioral problem-solving therapy protocol for individual cancer patients (Nezu et al. 1998) and a group problem-solving protocol successfully applied in patients with nonspecific low back pain (Van den Hout et al 2003; Kole-Snijders et al 2006). Participants learned to apply self-management skills in striving for personal goals (e.g. in work, household, hobbies, physical activity, family relationships and social contacts). Generalization to daily life during and after rehabilitation was promoted by practicing activities during sessions and by homework assignments (maximally 30 minutes weekly). Every session was structured in: 1) recapitulation of the previous week's session and exchanging daily life experiences; 2) discussing home assignments; 3) introducing new topics or problem-solving skills; 4) practicing problem-solving skills; 5) introducing the next homework assignments; and 6) relaxation exercises. Participants used a workbook containing a summary of the training, worksheets and assignments, as well as information on additional topics relevant to cancer patients. The first three weeks focused primarily on exchanging participants' experiences with cancer, interactive psycho-education about stress, relaxation, fatigue, exercise physiology, illness perceptions, as well as on promoting optimism and self-efficacy for self-management. From week 4 onward participants were primarily trained in applying problem-solving skills to realize personal goals by practicing the following steps in the circular problem-solving process: 1) problem orientation; 2) problem 
definition and formulation, including goal setting; 3) generation of alternative solutions (brainstorming); 4) decision-making; and 5) solution implementation and verification.

\section{Measures}

Socio-demographic and medical variables were assessed using self-report questionnaires, with confirmation of medical data by the referring physicians.

Implementation process was evaluated in several ways. A Case Record Form was completed after each session by exercise trainers and psychologists to monitor each participant's attendance (yes/no) and adverse events. Furthermore, in a Completeness Form psychologists rated after each CBT session the extent to which the session's elements were performed according to the protocol (1 - 6 scale from not at all to completely). Additionally, after each CBT session psychologists also monitored in the Case Record Form whether participants were involved in activities (yes/no) and whether they conducted their home assignments (yes/no). Finally, participants evaluated their appreciation of rehabilitation and its effectiveness ( $1-10$ scale from not at all to very much).

Problem-solving was measured using the SPSI-R (D'Zurilla et al. 2002; D'Zurilla and Nezu 2007; Kole-Snijders et al. 2006; Nezu 2004), a selfreport questionnaire. The SPSI-R, based on factor-analytic studies of the original SPSI, consists of five subscales: 1) negative problem orientation (10 items, range $0-40), 2$ ) positive problem orientation (5 items, range 0-20), 3) rational problem solving (20 items, range 0-80), 4) impulsivity/carelessness style (10 items, range 0-40) and 5) avoidance style (7 items, range 0-28). Higher scores on 1) negative problem orientation indicate the general tendency to view a problem as a significant threat to well-being, believe that problems are unsolvable (pessimism, negative outcome expectancies (Bandura 1997)), doubt one's personal ability to solve problems successfully (low-self-efficacy (Bandura 1997)), and become frustrated and upset when confronted with problems in living (low frustration tolerance). In contrast, higher scores on 2) positive problem orientation indicate the general tendency to appraise a problem as a challenge rather than a threat, believe that problems are solvable (optimism, positive outcome expectancies) (Bandura 1997), believe in one's personal ability to solve problems successfully (selfefficacy) (Bandura 1997), believe that successful problem-solving takes time, effort, and persistence, and commit oneself to solving problems with 
dispatch rather than avoidance. A high score on 3) rational problem solving style indicates that the person carefully and systematically gathers facts and information, identifies demands and obstacles, sets problem-solving goals (problem definition), generates a variety of different alternative solutions (generation of alternative solutions), evaluates possible consequences, judges and compares the alternatives, chooses (decision making) and, then, implements a solution while carefully monitoring and evaluating the outcomes (solution implementation and verification). A high score on 4) impulsivity/carelessness style indicates that a person considers only a few solution alternatives, often impulsively going with the first idea that comes to mind, scans alternatives and consequences quickly, carelessly and unsystematically, and monitors and evaluates solution outcomes carelessly and inadequately. A high score on 5) avoidance style indicates that a person prefers to avoid problems rather than confront them, puts off solving problems for as long as possible, waits for problems to resolve themselves, and attempts to shift the responsibility for solving his problem to others. Items are measured on 5-point Likert scales. Higher scores in positive problem orientation and rational problem solving and lower scores in the other scales are considered to reflect functional, constructive, adaptive and effective problem-solving (D'Zurilla et al. 2002). To obtain the total SPSI-R score (range 0 - 20), firstly, negative problem orientation, avoidance style and impulsivity/carelessness style are reversed-scored, secondly, each of the five subscales is divided by its number of items, and, finally, these scales are summed (D'Zurilla et al. 2002). In the present study Cronbach's $\alpha$ 's of the positive problem orientation were relatively low: 0.49 at baseline, 0.64 after rehabilitation, 0.66 at 3-month follow-up and 0.64 at 9-month follow-up. The Cronbach's $\alpha$ 's of the other scales were at least 0.83 at all measurement occasions, indicating good internal consistency (Nunnally 1978). These findings, except for those of positive problem orientation, reflect findings of other research (D'Zurilla et al 2002; Van den Hout et al. 2001). Reference scores of a sample of middle-aged adults living in the community $(\mathrm{n}=100$, age $46.5 \pm 4.7,70 \%$ females) are: positive problem orientation $13.5 \pm 3.9$; rational problem-solving $47.9 \pm 15.1$; negative problem orientation 9.5 4.0 ; impulsivity $9.1 \pm 6.0$; avoidance $6.3 \pm 5.9$; total problem-solving $14.4 \pm 3.0$ (D'Zurilla et al 2002).

Distress was measured using the Hospital Anxiety and Depression Scale (HADS) (Spinhoven et al. 1997; Van Hemert and Ormel 1996; Zigmond and Snaith 1983), a self-report questionnaire of anxiety and depression. Because items that may have a physical cause (e.g. insomnia or weight loss) are not 
included in this questionnaire, the HADS is considered to be unbiased by coexisting general medical conditions (Spinhoven et al. 1997). The total scale (range 0 - 42), assessing general distress or negative affect, contains two 7-item subscales: anxiety (range 0 - 21) and depression (range 0 - 21). Items are measured on 4-point Likert scales. Higher scores reflect higher levels of anxiety and depression. In the present study Cronbach's $\alpha$ 's were at least 0.81 at all measurement occasions, reflecting previous scale findings (Spinhoven et al. 1997), and indicating good internal consistency (Nunnally 1978). Reference scores of a random sample of the general Dutch population $(\mathrm{n}=199$, age $39.9 \pm 12.4$ years, $53.8 \%$ females) are: anxiety $5.1 \pm 3.6$; depression 3.4 \pm 3.3 ; total scale $8.4 \pm 6.3$. Reference scores of Dutch psychiatric outpatients $(\mathrm{n}=491$, age $35.6 \pm 11.2,61.9 \%$ females $)$ are: anxiety $11.1 \pm 4.7$; depression 9.3 \pm 5.2 ; total scale 20.4. \pm 8.9 (Spinhoven et al. 1997). In the present study participants with anxiety scores $\geq 11$ and/or with depression scores $\geq 11$ were classified as higher distressed, according to the cut-off scores for "definite cases" of anxious or depressive symptoms in the HADS manual (Van Hemert and Ormel 1996), and those with anxiety and depression scores $<11$ were classified as participants with lower distress ("doubtful" or "non-cases" according the HADS manual).

\section{Statistical analysis}

A priori power analysis for a comparison between the randomized groups on the primary outcome anxiety estimated a sample size of 64 participants in each group to detect a moderate effect-size $(\mathrm{d}=0.50)$ with a power of 0.80 and a two-tailed alpha of 0.05 . Accounting for an estimated dropout of $10 \%$, 71 participants in each group were needed.

Statistical analysis was performed using SPSS statistical software, version13.0, 2004. For testing hypotheses 1 and 2 longitudinal intention-to-treat analyses were conducted, using mixed linear regression models (Blackwell et al. 2006). In these analyses the program accounts for missing data based on the observed data. The rehabilitation effect on problem-solving, both anxiety and depression was tested in PT $+\mathrm{CBT}$ as compared to PT immediately after rehabilitation, and at three and nine months follow-up. Level 1 was time, level 2 was participant. Center was also included as a factor because of the four-center study design. Furthermore, we included a covariate "pre-rehabilitation distress" (higher, lower) and interaction terms "prerehabilitation distress*time" and "pre-rehabilitation distress* rehabilitation group". 
Effect sizes (ES) were calculated as indices measuring the magnitude of a treatment effect. An ES $<0.2$ reflects "no effect," ES $\geq 0.2,<0.5$ "small effect," ES $\geq 0.5, \leq 0.8$ "moderate effect" and ES $\geq 0.8$ "large effect" (Cohen 1988).

For testing hypothesis 3 , firstly, for each of the anxiety and depression scales a multiple linear regression analysis was conducted to assess whether participants' baseline scores in these outcomes were correlated with their baseline scores in the problem-solving scales, while adjusting for center. Secondly, for each of the anxiety and depression scales a multiple linear regression analysis was conducted to assess whether participants' pre-to-post rehabilitation changes in these scales were correlated with pre-to-post rehabilitation changes in the problem-solving scales. Dependent variables were the post-rehabilitation scores of the anxiety and depression scales, respectively, independent variables were the post-rehabilitation scores of the problem-solving scales, and we corrected for baseline scores of these dependent and independent variables and for center. Assumptions regarding multicollinearity and multiple linear regression analyses were met. Significant partial correlations (r) between predictors and outcomes, controlling for all other predictors in the model, are presented.

Differences were explored in baseline socio-demographic and medical characteristics between included patients and those who met the inclusion criteria but refused participation or no longer felt a need for rehabilitation at baseline as well as between participants who stayed in the study and those who discontinued rehabilitation. In addition, differences were tested between PT and PT $+\mathrm{CBT}$ and between participants with higher and those with lower pre-rehabilitation distress in socio-demographic and medical characteristics, in process variables, and in baseline problem-solving and distress. ANOVA was used for continuous data and Chi-Square tests were used for categorical data. 


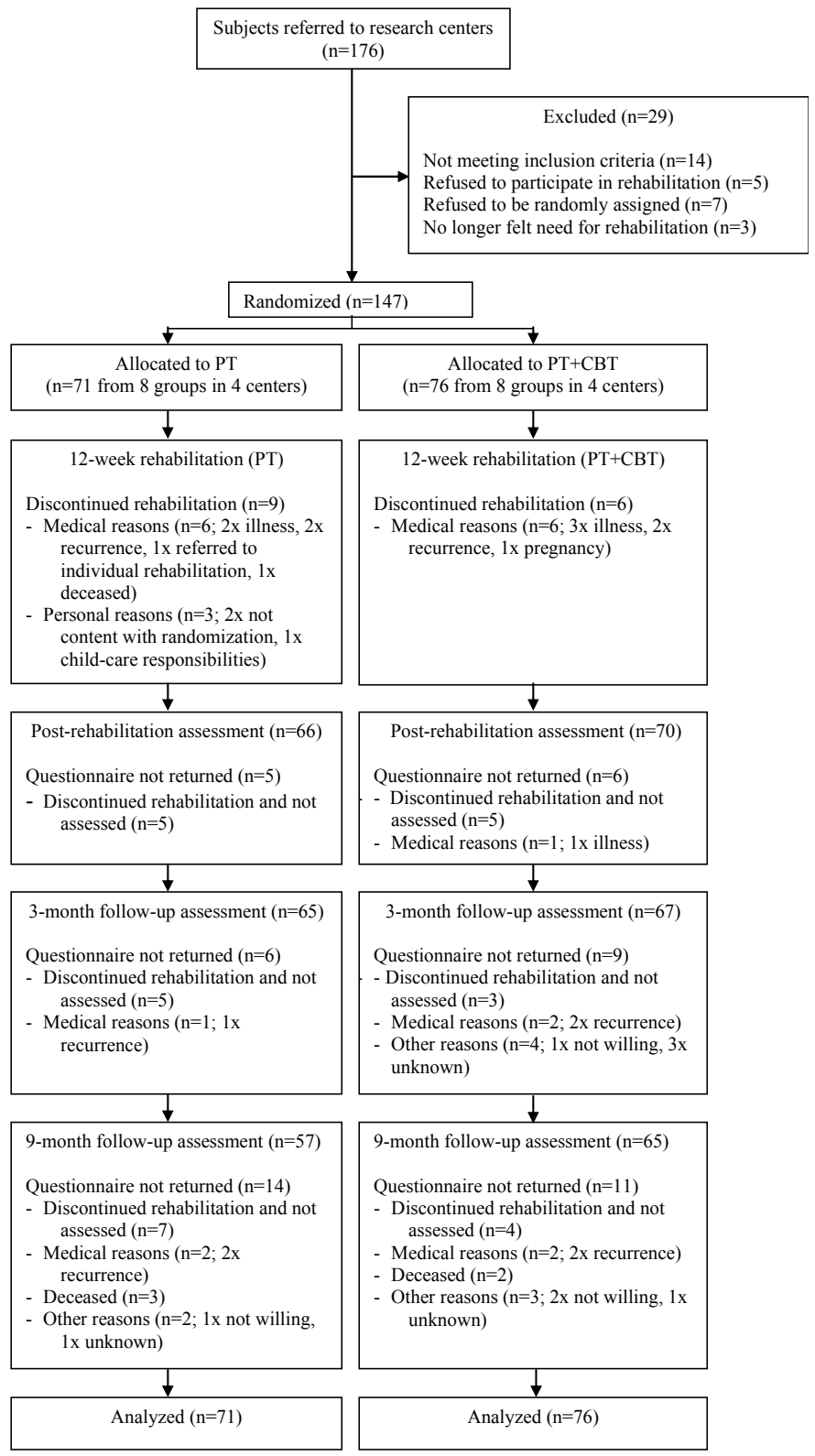

Figure 1. Flow of the participants through the study Abbreviations: PT: physical training; PT+CBT: physical training plus cognitive-behavioral training. 


\section{Results}

No differences were found in baseline socio-demographic and medical characteristics comparing the 147 included patients to the 15 patients who met the inclusion criteria, but refused participation or no longer felt a need for rehabilitation at baseline, except that included patients received less often radiotherapy $(57.1 \%$ versus $86.7 \%$, $\mathrm{p}$-value $=0.03)($ Table 1$)$. No differences were found between the 132 participants who stayed in the study and the 15 participants who discontinued rehabilitation.

\section{Patient characteristics}

Groups were balanced in all socio-demographic and medical variables (Table 1, p-values $>0.05$ ). At baseline, less than one third of the participants were higher distressed (HADS anxiety $\geq 11$ and/or HADS depression $\geq 11$; PT: $\mathrm{n}=19(26.8 \%)$; PT+CBT: $\mathrm{n}=24(31.6 \%)(\mathrm{p}$-value $=0.52))$. Initially higher distressed participants did not differ from initially lower distressed participants in socio-demographic and medical variables, except that in $\mathrm{PT}+\mathrm{CBT}$ initially higher distressed participants were older $(51.7$ years \pm 10 versus 45.9 years \pm 10.3$)$ and were less often employed at diagnosis $(50 \%$ versus $80.8 \%$ ) than their counterparts with lower distress ( $\mathrm{p}$-values $<0.05$ ).

\section{Process}

Participants in PT and in PT + CBT completed $83.5 \%$ of 24 physical training sessions (PT 20 $\pm 5.2 ; \mathrm{PT}+\mathrm{CBT} 20 \pm 4.7$ ) and $\mathrm{PT}+\mathrm{CBT}$ participants completed

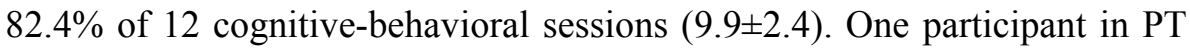
collapsed during rehabilitation and died at the first-aid station, which, after autopsy, the physician diagnosed as not related to the intervention. No further adverse events were reported.

The psychologists monitored the extent to which they performed the CBT sessions' elements according to protocol: the median of overall performance during 12 sessions in 8 groups was 4.5 ( \pm 0.8 , range 3.3 to 5.6, scale 1 - 6 from not at all to completely). CBT participants were active in $75.4 \%$ of 12 sessions $(9.1 \pm 2.5)$ and they completed their home assignments in $56.7 \%$ of the sessions $(6.8 \pm 3.3)$.

After rehabilitation, $31.3 \%$ of PT participants stated that they would have preferred to receive $\mathrm{PT}+\mathrm{CBT}$ and $81.4 \%$ of the $\mathrm{PT}+\mathrm{CBT}$ participants rather 
received $\mathrm{PT}+\mathrm{CBT}$ than $\mathrm{PT}$. These figures differed significantly between $\mathrm{PT}$ and PT + CBT ( -values $<0.05$ ). PT participants rated their appreciation of rehabilitation with $8.4 \pm 1.1$ and its effectiveness with $7.9 \pm 1.6$ and $\mathrm{PT}+\mathrm{CBT}$ participants rated their appreciation of rehabilitation with $8.6 \pm 1.0$ and its effectiveness with $8.3 \pm 1.3$ ( 0 - 10 scale from not at all to very much). These figures did not significantly differ between PT and PT + CBT (p-values $>$ 0.05). No significant differences between initially higher distressed and initially lower distressed participants were revealed on any of the process measures ( $p$-values $>0.05$ ).

Table 1. Baseline characteristics ${ }^{a}$

\begin{tabular}{|c|c|c|c|}
\hline & $\begin{array}{l}\text { PT } \\
n=71\end{array}$ & $\begin{array}{l}\mathrm{PT}+\mathrm{CBT} \\
\mathrm{n}=76\end{array}$ & $\begin{array}{l}\text { Overall } \\
n=147\end{array}$ \\
\hline Age (years) & $49.9 \pm 11.3$ & $47.8 \pm 10.5$ & $48.8 \pm 10.9$ \\
\hline \multicolumn{4}{|l|}{ Sex } \\
\hline Female & $57(80.3)$ & $66(86.8)$ & $123(83.7)$ \\
\hline \multicolumn{4}{|l|}{ Educational level } \\
\hline Low & $14(19.7)$ & $6(7.9)$ & $20(13.6)$ \\
\hline Middle & $32(45.1)$ & $40(52.6)$ & $72(49.0)$ \\
\hline High & $25(35.2)$ & $30(39.5)$ & $55(37.4)$ \\
\hline \multicolumn{4}{|l|}{ Marital status } \\
\hline Married/living together & $51(71.8)$ & $53(69.7)$ & $104(70.7)$ \\
\hline \multicolumn{4}{|l|}{ Employment status } \\
\hline Employed at diagnosis & $53(74.6)$ & $54(71.1)$ & $107(72.8)$ \\
\hline Actually working at baseline & $23(32.4)$ & $32(42.1)$ & $55(37.4)$ \\
\hline \multicolumn{4}{|l|}{ Cancer type } \\
\hline Breast & $34(47.9)$ & 48 (63.2) & $82(55.8)$ \\
\hline Hematological & $8(11.3)$ & $15(19.7)$ & $23(15.6)$ \\
\hline Gynecological & $11(15.5)$ & $6(7.9)$ & $17(11.6)$ \\
\hline Urologic & $6(8.5)$ & $3(3.9)$ & $9(6.1)$ \\
\hline Lung & $2(2.8)$ & $2(2.6)$ & $4(2.7)$ \\
\hline Colon & $2(2.8)$ & $1(1.3)$ & $3(2.0)$ \\
\hline Other & $8(11.3)$ & $1(1.3)$ & $9(6.1)$ \\
\hline \multicolumn{4}{|l|}{ Treatment $>3$ months ago } \\
\hline Surgery & $62(87.3)$ & $64(84.2)$ & $126(85.7)$ \\
\hline Chemotherapy & $45(63.4)$ & $55(72.4)$ & $100(68.0)$ \\
\hline Radiotherapy & $41(57.7)$ & $43(56.6)$ & $84(57.1)$ \\
\hline Time since treatment (years) & $1.4 \pm 2.1$ & $1.2 \pm 1.3$ & $1.3 \pm 1.7$ \\
\hline \multicolumn{4}{|l|}{ Distress at start } \\
\hline HADS total score & $12.9(7.6)$ & $13.9(7.8)$ & $13.4(7.7)$ \\
\hline \multicolumn{4}{|l|}{ Problem solving at start } \\
\hline SPSI-R total score & $12.5(2.5)$ & $12.1(2.2)$ & $12.3(2.3)$ \\
\hline
\end{tabular}

Abbreviations: PT: physical training; PT+CBT: physical plus cognitive-behavioral training. a Continuous data: mean \pm standard deviation; categorical data: frequency (percentage). 


\section{Pre-rehabilitation levels of problem-solving, anxiety and depression}

At the start of rehabilitation, $\mathrm{PT}$ and $\mathrm{PT}+\mathrm{CBT}$ were balanced in all domains of problem-solving and distress (Table 2, p-values $>0.05$ ). Participants performed significantly worse in almost all domains of problem-solving, except in rational problem-solving, and in anxiety and depression than did reference groups in general population samples (see for reference scores section "Measures"; p-values < 0.05). Furthermore, participants reported significantly better total problem-solving than did participants in the problem-solving training for distressed cancer patients of Nezu and colleagues (2003) (Table 1; total problem-solving $12.3 \pm 2.3$ versus $8.7 \pm 1.8$ ). Additionally, participants in the current study reported less anxiety and depression than Dutch psychiatric out-patients (Spinhoven et al. 1997) (p-values < 0.05). At baseline, higher distressed participants in PT and PT $+\mathrm{CBT}$ showed more negative problem orientation, anxiety, and depression than participants with lower distress ( $\mathrm{p}$-values $<0.05$ ), which was in accordance with our expectations. However, contrary to our expectations, initially higher distressed participants in PT and $\mathrm{PT}+\mathrm{CBT}$ did not significantly differ from initially lower distressed participants in the other domains of problemsolving ( $p$-values $>0.05$ ). At baseline, within the subgroups of higher distressed participants and participants with lower distress no significant differences were found between PT and PT $+\mathrm{CBT}$ in any domain of problemsolving and distress ( $\mathrm{p}$-values $>0.05)$.

\section{Changes in problem-solving, anxiety and depression}

Participants in $\mathrm{PT}+\mathrm{CBT}$ showed no significant changes beyond those of participants in PT in any domain of problem-solving or distress (between group-changes, Table 2).

Moreover, initially higher distressed participants did not profit more from adding CBT to PT than initially lower distressed participants in any domain of problem-solving or distress (interactions pre-rehabilitation distress*rehabilitation group: all p-values $>0.05)$.

After rehabilitation, participants in $\mathrm{PT}$ and $\mathrm{PT}+\mathrm{CBT}$ reported significant, small to moderate (Cohen 1988), reductions in negative problem orientation (ES 0.24 and 0.33 , respectively), anxiety (ES 0.55 and 0.45 , respectively), and depression (ES 0.49 and 0.44, respectively) and maintained these reductions at three and nine months follow-up (within-group changes, Table 2). After rehabilitation, participants in $\mathrm{PT}$ and in $\mathrm{PT}+\mathrm{CBT}$ were at the level of the general population in two domains of problem-solving, positive 


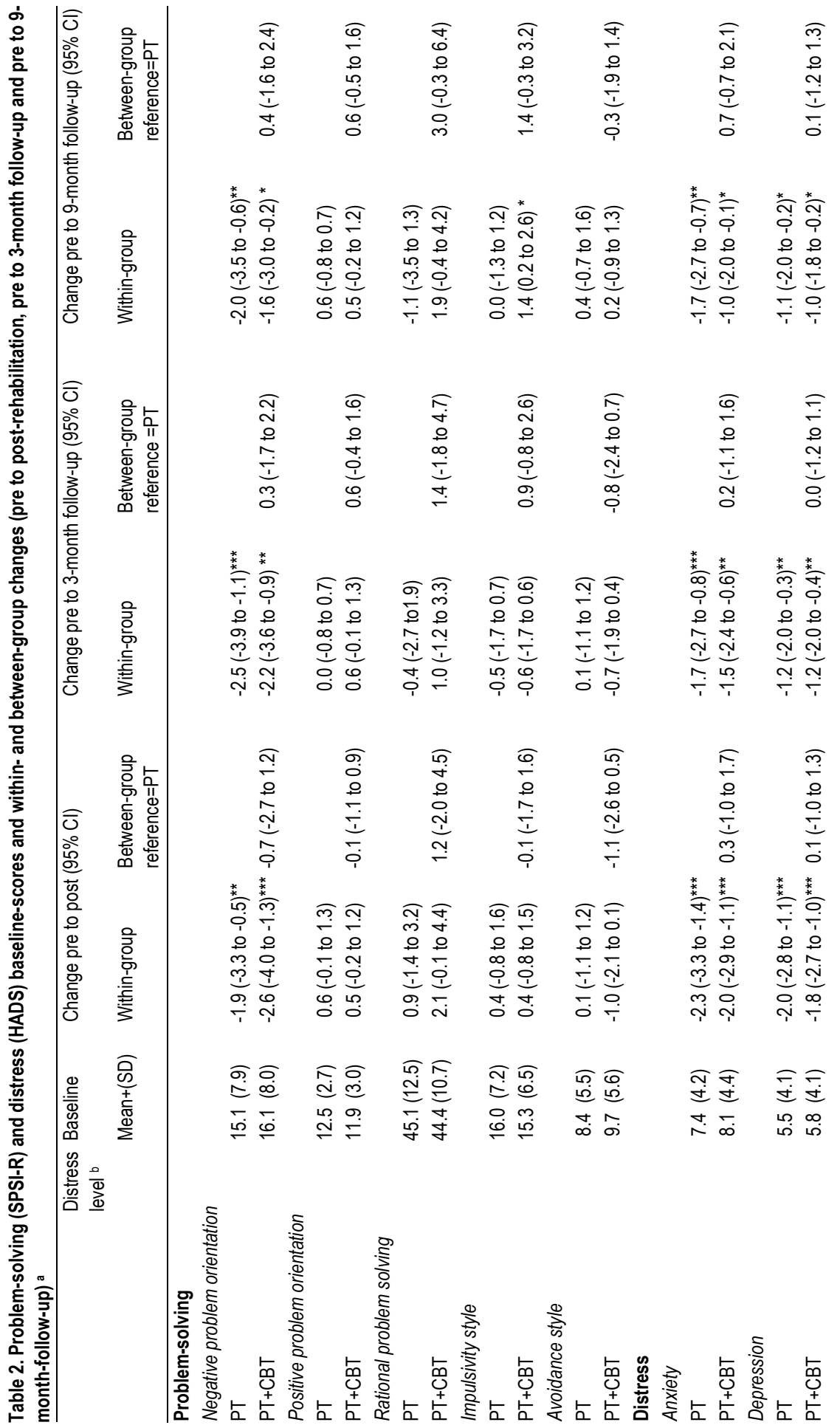




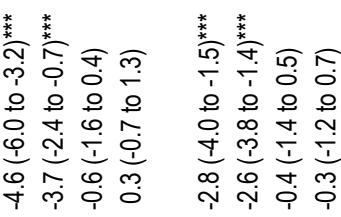

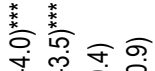

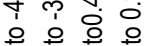

7.00

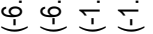

审守审审

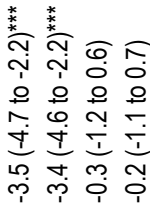

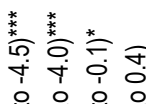

우 운우운

I

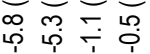

क $\widehat{\triangleleft} \propto$

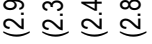

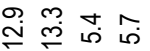

离 흘

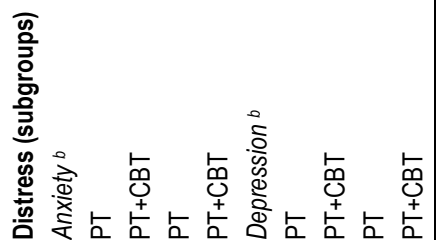

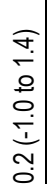

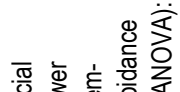

䂞

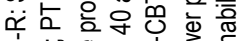

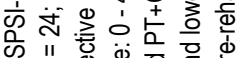

我 "

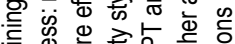

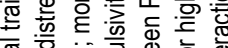

징

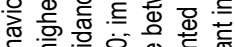

क्ष

di

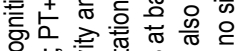

ठ

謇 "

बi

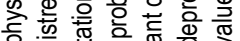

응 흔.

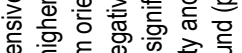

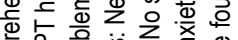

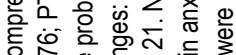

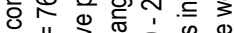

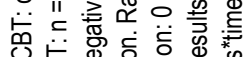

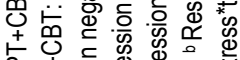

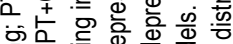

잉

둔

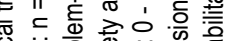

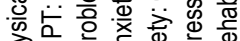

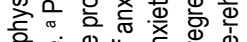

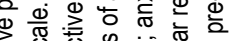

के

类.

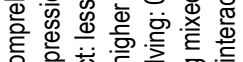

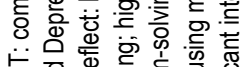

는 훙

需

产离

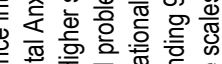

政产

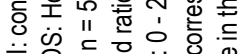

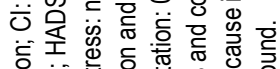

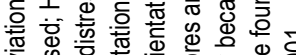

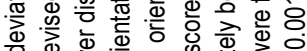
은 先

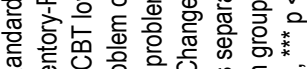
要

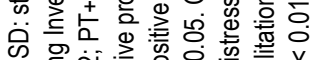

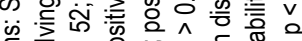

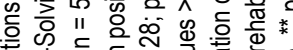

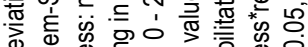

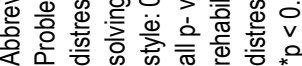


problem orientation and rational problem-solving, and remained at these levels at 3-month follow-up (p-values > 0.05). Moreover, participants in $\mathrm{PT}+\mathrm{CBT}$ remained at the general population level in rational problemsolving at 9-month follow-up (p-values $>0.05$ ). Anxiety and depression were decreased in $\mathrm{PT}$ and $\mathrm{PT}+\mathrm{CBT}$ to general population levels after rehabilitation ( $\mathrm{p}$-values $>0.05)$. In PT anxiety and depression remained at these levels at three and nine months follow-up, whereas in PT+CBT only depression remained at the general population level at 3-month follow-up ( $\mathrm{p}$ values $>0.05$ ). Initially higher distressed participants did not differ from initially lower distressed participants in changes in problem-solving (prerehabilitation distress*time interactions: all p-values $>0.05$ ). Initially higher distressed participants in $\mathrm{PT}$ and $\mathrm{PT}+\mathrm{CBT}$ showed significant reductions in anxiety and depression after rehabilitation and these reductions were still maintained at 9-month follow-up ( $p$-values $<0.05$ ), but they did not yet reach the general population's levels (p-values $>0.05$ ). Initially lower distressed participants showed significant reductions in anxiety (PT) and depression ( $\mathrm{PT}$ and $\mathrm{PT}+\mathrm{CBT}$ ) after rehabilitation (p-values $<0.05$ ), but these reductions were not maintained. Anxiety and depression of these initially lower distressed participants were at the general population's levels at all measurement occasions ( $\mathrm{p}$-values $>0.05$ )

\section{Associations between problem-solving and anxiety and depression}

At the start of rehabilitation, more anxiety of participants was associated with more negative problem orientation $(\mathrm{r}=0.47)$ and with less avoidance $(\mathrm{r}$ $=0.17)$, more depression was associated with more negative problem orientation $(\mathrm{r}=0.30)$ ( $\mathrm{p}$-values $<0.05)$. After rehabilitation, participants' reduced anxiety and reduced depression were associated with reduced negative problem orientation $(\mathrm{r}=0.36 ; \mathrm{r}=0.31$, respectively) and with increased impulsivity $(\mathrm{r}=0.25 ; \mathrm{r}=0.29$, respectively) ( $\mathrm{p}$-values $<0.05$ ). None of the other problem-solving scales were significantly correlated with anxiety or depression ( $\mathrm{p}$-values $>0.05$ ).

\section{Discussion}

This four-center trial was the first study to investigate short and long-term effects of a comprehensive physical training program and the additional value of cognitive-behavioral training on cancer survivors' problem-solving 
and distress. Our first hypothesis, that $\mathrm{PT}+\mathrm{CBT}$ would show more short and long-term positive effects on problem-solving, anxiety and depression, than PT, was not supported. Furthermore, no support was found for our second hypothesis that initially higher distressed participants would profit more from adding CBT to PT than participants with lower pre-rehabilitation distress. After rehabilitation, participants in both PT and in PT $+\mathrm{CBT}$ showed significant small to moderate reductions in negative problem orientation, anxiety and depression, which were maintained at three and nine months after rehabilitation. Initially higher distressed participants did not differ from initially lower distressed participants in changes in problem-solving. Initially lower distressed participants showed significant reductions in anxiety (PT) and depression $(\mathrm{PT}$ and $\mathrm{PT}+\mathrm{CBT})$ after rehabilitation, but these reductions were not maintained. Initially higher distressed participants in PT and $\mathrm{PT}+\mathrm{CBT}$ showed significant reductions in anxiety and depression after rehabilitation. These reductions were maintained up to 9-month follow-up. Our third hypothesis, that improvements in problem-solving were associated with improvements in anxiety and depression, was partly supported by significant associations between reductions in negative problem orientation and reductions in anxiety and depression.

Our study confirmed the beneficial effects of physical training interventions on cancer survivors' distress (Conn et al. 2006; Daley at al. 2007; McNeely et al. 2006; Milne et al. 2007; Schmitz et al. 2005; Segar et al. 1998). Unique is that we showed positive effects on distress of a physical training intervention for a longer follow-up period (9 months) in a mixed group of cancer survivors. Until now only short-term follow-up studies (10-week (Segar et al. 1998), 3-month follow-up (Milne et al. 2007) and 18 week-follow-up (Daley et al. 2007)) in breast cancer survivors showed beneficial effects of physical training on anxiety and/or depression. The benefits of comprehensive physical training might, firstly, be explained by its significant effects on physical fitness (May et al. 2008). An additional explanation might well be the integrated self-management approach, which might have reduced negative problem orientation, which is related to increased self-efficacy and more positive outcome expectancies (Bandura 1997; D'Zurilla et al. 2002; Nezu et al. 2004). A final explanation might be the group-format, providing opportunities for cooperation within the group, which was positively related to improved physical functioning and quality of life (May et al. 2007a).

Contrary to our expectations, CBT did not have additional value. This can hardly be explained by flaws in the delivery of CBT considering the experienced therapists trained to apply the standardized protocols, good fidelity 
and completeness, high attendance and low drop-out rates. More plausible explanations are the relatively mild complaints of participants at the start of rehabilitation combined with significant and durable benefits of comprehensive physical training, with the result that after rehabilitation participants were at general population levels with respect to positive problem orientation, rational problem-solving, anxiety and depression. Initially higher distressed participants profited most, which is important and desirable as rehabilitation aims to support those cancer survivors experiencing problems. The fact that adding CBT did not have additional effects for distress reduction does not allow the conclusion that CBT by itself may not reduce distress in cancer survivors as we did not include a CBT-only intervention in our design. Future research might show whether the large benefits of problemsolving training in highly distressed cancer patients reported by Nezu et al. (2003) could be replicated in highly distressed cancer survivors in the Netherlands. The long-term beneficial effects on negative problem orientation and distress of comprehensive physical training in a mixed group of cancer survivors are promising. These findings are especially relevant for the cancer survivors who usually enroll in rehabilitation which is offered in more than 60 centers in The Netherlands. These cancer survivors, who apply for rehabilitation on their own initiative, meet selection criteria comparable to ours. Due to the fact that cancer survivors are highly motivated to attend an exercise intervention (Stull et al. 2007) and that, as mentioned, alternatives are available in the Netherlands we were not able to include a randomized non intervention control group. A further limitation of our study was the rather low internal consistency of the positive problem orientation scale. Strengths of our study were the good internal consistency of all other measurement instruments, the length of the follow-up period, the large sample size, high attendance and low drop-out rates.

In conclusion, comprehensive physical training had significant and durable positive effects on cancer survivors' negative problem orientation, as well as on anxiety and depression. Adding cognitive-behavioral training did not add to these beneficial effects, neither in the short-term nor in the long-term. 
Chapter 7

General discussion 
The main aims of this dissertation were, firstly, to investigate the effects of multi-modal physical and psychosocial rehabilitation on cancer survivors' quality of life and, secondly, to investigate whether multi-modal physical and psychosocial rehabilitation was more effective than single-focused physical rehabilitation. Two cancer rehabilitation programs were analyzed. Firstly, the Dutch 12-week group-based rehabilitation program "Recovery \& Stability", which consisted of physical training sessions (twice weekly), guided by physiotherapists, and 7 psychosocial sessions, guided by different professionals addressing different themes. Lastly, the 12-week group-based rehabilitation program "OncoRev" in which physical training sessions (twice weekly) as well as psychosocial training sessions (once weekly) were based on standardized self-management protocols and guided by therapists. In the trial investigating the "OncoRev" program, single focused rehabilitation consisted of comprehensive self-management physical training and multimodal rehabilitation contained comprehensive self-management physical training as well as self-management cognitive-behavioral training. This general discussion starts with presenting the main findings of our studies, followed by theoretical reflections on the rehabilitation programs and methodological reflections on the study designs. Next, this chapter addresses implications for practice and future research. Lastly, this chapter presents a final conclusion.

\section{Main findings}

After participation in the multi-modal "Recovery \& Stability" program, cancer survivors reported significant improvements in all domains of quality of life (chapter 2). However, these improvements were clinically relevant only for 2 subgroups of participants in three domains of quality of life, i.e. for non-working participants in role functioning, and for participants with other cancer diagnoses than breast cancer in physical functioning and social functioning. Participants in the "Recovery \& Stability" program valued rehabilitation as an important stepping stone in their ongoing recovery process (chapter 3). Furthermore, they saw the combination of physical and psychosocial sessions and the group-format as strengths of the program and suggested that the program should focus more on developing skills to cope with cancer (chapter 3). A new program was developed and tested in the "OncoRev" trial that investigated the effects of multi-modal rehabilitation and single focused rehabilitation on cancer survivors' quality of life, prob- 
lem-solving and distress immediately after rehabilitation and at three and nine months after rehabilitation (chapters 4, 5, and 6). Immediately after rehabilitation participants in the rehabilitation groups showed significant clinically relevant improvements, predominantly in physical domains of quality of life, i.e. reduced role limitations due to physical problems, better physical functioning, and improved vitality and health change (chapter 4) as compared with waiting-list participants with no intervention. The effects on cancer survivors' quality of life of multi-modal rehabilitation did not significantly differ from those of single focused rehabilitation at any of the followup measurements (chapters 4 and 5). Compared with pre-rehabilitation, participants in the rehabilitation groups reported significant improvements in all domains of quality of life at all follow-up measurements (chapters 4 and 5). These improvements remained clinically relevant in almost all domains of quality of life at 9 months after rehabilitation (chapter 5). Compared with pre-rehabilitation, participants in the rehabilitation groups also showed significant reductions in one domain of problem-solving, i.e. negative problem orientation, and in distress, i.e. anxiety and depression, at all follow-up measurements (chapter 6). Furthermore, also in problem-solving and distress multi-modal rehabilitation did not outperform single focused rehabilitation. Subsequent detailed analysis of the effects of the interventions on participants with higher levels of pre-rehabilitation distress compared with participants with lower pre-rehabilitation distress showed no difference in benefits in problem solving and distress at any of the follow-up measurements (chapter 6).

The "OncoRev" single focused training had substantial and durable beneficial effects for quality of life, distress and one domain of problem-solving, i.e. negative problem orientation. Combining this training with cognitivebehavioral training did not have additional value, neither in the short-term, nor in the long-term.

\section{Theoretical reflections}

The participants in the "OncoRev" program reported clinically relevant improvements in more domains of quality of life than the participants in the "Recovery \& Stability" program (chapters 2 and 5). Thus, in accordance with our expectations, the "OncoRev" self-management rehabilitation program seemed more effective than the "Recovery \& Stability" program. However, contrary to our expectations, multi-modal rehabilitation did not 
lead to benefits beyond those of single focused rehabilitation (chapters 4, 5, and 6). The theories applied in this dissertation might help to understand these findings.

\section{Biopsychosocial model}

Combining physical training and psychosocial training in both rehabilitation programs was based on the biopsychosocial model (Engel 1977; Engel 1980; Engel 1997) that implies that physical and psychosocial interventions might each affect both physical and psychosocial problems, and that when combined these interventions might enhance the effect of the entire intervention. As the "Recovery \& Stability" study did not compare the separate effects of the physical and psychosocial modules (chapter 2), it was not possible to investigate whether multi-modal rehabilitation was more effective than single focused rehabilitation. Participants in the focus groups stated that although they were primarily attracted to physical rehabilitation, they had appreciated that "Recovery \& Stability" contained physical as well as psychosocial sessions (chapter 3). The "OncoRev" trial evaluated multimodal as well as single focused physical rehabilitation, but the separate effects of cognitive-behavioral rehabilitation were not investigated (chapters 4,5 , and 6). Therefore, although this study showed that adding cognitivebehavioral training to physical training did not have additional value this trial does not allow the conclusion that cognitive-behavioral training by itself may not have beneficial effects. Participants in multi-modal as well as in single focused rehabilitation valued the rehabilitation they received (chapter 6). After rehabilitation, more than eighty percent of the participants in multimodal rehabilitation would not have wanted to receive single focused training and one third of participants in single focused training would have preferred multi-modal rehabilitation (chapter 6). Thus, although cognitivebehavioral training did not have additional value, many participants in cancer rehabilitation felt that physical as well as psychosocial interventions were necessary to fully address their needs. In fact, integrating selfmanagement within physical training, extensively described by van Weert and colleagues (2008), may have addressed these needs to such a level that adding cognitive-behavioral training did not have a measurable surplus value. By including self-management in physical training this physical training in itself may well have become an adequate biopsychosocial intervention. This explanation should be further investigated and other possible explanations need to be considered too. 


\section{Social comparison, social support, and modeling}

The group-format and group processes in rehabilitation may also have contributed to the beneficial effects of rehabilitation and to the fact that multi-modal rehabilitation was not more effective than single focused rehabilitation. The group-format provided opportunities for social comparison (Bogart and Helgeson 2000; Festinger 1954; Stanton et al. 1999), social support (Helgeson and Cohen 1996) and modeling (Bandura 1986). Participants in the focus groups appreciated the patient-to-patient contacts in both modules of "Recovery \& Stability", because they felt that participants supported each other by sharing experiences, worries, humor and striving for physical and mental improvement (chapter 3). They mentioned experiences in both modules that were examples of downward and upward social comparisons (Stanton et al. 1999; Taylor and Brown 1988; Taylor and Lobel 1989), social support (Helgeson and Cohen 1996) and modeling (Bandura 1986). The group-format may well have supported participants in reaching physical and mental improvements by enabling them to work together in striving toward their individual goals. This notion was supported by a recent study on the role of group cohesion in the "OncoRev" program (May et al. 2007a). This study showed that higher ratings of cooperation within the group predicted better post-rehabilitation global quality of life and physical functioning in men and women and also less fatigue in men.

\section{Social cognitive theory, self-efficacy, and self-management}

It has been shown that including more social cognitive components, such as modeling and self-efficacy enhancing techniques and a self-management approach in an intervention had positive effects on cancer patients' quality of life (Graves 2003). Therefore, also the self-management approach (Creer 2000; Holroyd and Creer 1986; Mesters et al. 2002), including the applied self-efficacy enhancing techniques (Bandura 1997) in both "Oncorev" modules may explain why participants in single focused rehabilitation in the "OncoRev" program showed greater improvements in quality of life (chapter

5) than participants in multi-modal "Recovery \& Stability" rehabilitation (chapter 2).

\section{Problem-solving model of stress and coping}

The circular problem-solving process, in which people manage and adapt to stressful life events, is highly comparable to the circular self-management 
process (chapter 1). Therefore, measures of problem-solving might, besides evaluating changes in specific problem-solving domains, also reflect changes in self-management of participants. In cancer patients particularly more negative problem orientation was correlated with more anxiety and depression, and in cancer survivors more negative problem orientation and less rational problem-solving predicted more anxiety and depression years after medical treatment (Nezu et al. 1999a; Nezu 2004). The "OncoRev" trial showed that, before rehabilitation negative problem orientation was associated with anxiety and depression and, that after rehabilitation reduced negative problem orientation was associated with reduced anxiety and depression (chapter 6). This implies that in cancer rehabilitation negative problem orientation is the most relevant problem-solving domain. The associations of problem orientation with problem-focused and emotionfocused coping and cognitive appraisal (Lazarus 1993), self-efficacy and outcome-expectancies (Bandura 1997) reported by others (D'Zurilla and Nezu 2007; Nezu 2004) support the notion that integrating self-management in physical training may have contributed to its benefits for participants' quality of life and distress.

\section{Methodological reflections}

\section{Recruitment}

"Recovery \& Stability" and "OncoRev" focused on cancer survivors who had successfully completed their primary medical treatment, but were still struggling with physical, psychological, and/or social problems. Cancer survivors received information about the available rehabilitation programs from, for instance, family, friends, physicians, or news media. Ideally, patients in need for rehabilitation are referred by their doctors. In practice, many patients who are able and willing to enter such a program apply on their own initiative, and then are requested to obtain their doctor's referral to check the selection criteria. At the time of the start of "Recovery \& Stability" few cancer survivors were aware of existing opportunities for cancer rehabilitation. Although cancer rehabilitation is now being offered in more than sixty Dutch centers, unfortunately, rehabilitation seems not yet accessible for all those in need. One reason might be that the costs of rehabilitation are still not covered by all health insurance companies. Furthermore, physicians do not systematically screen cancer patients for rehabilitation needs 
and do not regularly refer those in need for rehabilitation. Those who were most in need for rehabilitation might have been less able to seek for help because of the impact of their problems. Thus, our studies included cancer survivors with physical, psychological and/or social problems, but may not have been easily accessible for those who were most in need of rehabilitation.

\section{Internal validity}

Rehabilitation protocols and process-evaluation. The physical module in the "Recovery \& Stability" program followed a fairly detailed protocol for each session and the physical training sessions were guided by two expert physiotherapists (chapter 3). However, the psychosocial sessions in "Recovery \& Stability" were guided by a variety of different professionals, and the psychosocial protocol only determined for each session the theme and the health care professionals, and provided in broad outline instructions for these sessions (chapter 3). Another limitation in this study was the lack of a process-evaluation. Therefore, it can not be ruled out that "OncoRev" showed more clinically relevant improvements in quality of life than "Recovery \& Stability" because of flaws in the delivery of "Recovery \& Stability". The "OncoRev" study, included detailed protocols for both modules delivered by carefully trained therapists, and a process-evaluation showing good fidelity and completeness of the interventions that enhanced the internal validity of this study (chapter 4,5 , and 6). Therefore, the lack of additional value of cognitive-behavioral training can hardly be attributed to flaws in the delivery of this training.

Quantitative and qualitative assessments. The quantitative studies all applied validated self-report questionnaires. Furthermore, in the "OncoRev" study quality of life was measured using a cancer-specific questionnaire (chapter 4) as well as with a general health-related questionnaire (chapter 5). The fact that the results of both these questionnaires were consistent with each other, enhance the validity of the findings in our studies. Self-report of quality of life is adequate as the experience of quality of life is subjective and is best evaluated by the respondents themselves. This also holds for the measures of distress and to some extent for problem-solving, although one might argue that assessments such as clinician-rated distress assessments and performance tests for problem-solving might have enhanced the validity of these measures (D'Zurilla and Nezu 2007). We did not assess or control for possible social desirability bias. However, since the subjective self-reported 
improvements in physical functioning (chapters 4 and 5) were confirmed by significant clinically relevant improvements in physical fitness measured by objective physical tests (May et al. 2008), social desirability bias may have been limited. In addition to the quantitative evaluation of "Recovery \& Stability" the qualitative focus group study offered in-depth insights in the views of the participants on the value of rehabilitation, the group-format, and the separate modules, which supported further development of the rehabilitation program.

Sample sizes and drop-out rates. The sample size in the "Stability and Recovery" study was large, and the sample sizes in the focus group study and the "OncoRev" study were adequate for answering the main research questions. Drop-out rates of participants in the "Recovery \& Stability" study and in the "OncoRev" study were low. Reasons for dropping out from the "Recovery \& Stability" program were not recorded due to logistic restrictions. Dropout from the "OncoRev" program was mostly due to medical reasons, such as recurrences. Considering that some dropout from a cancer rehabilitation program due to a recurrence or a new cancer diagnosis is inevitable and given the mostly medical reasons for dropping out from "OncoRev", it seems plausible that dropping out from "Recovery and Balance" was at least partially due to medical reasons. This indicates that participants in both programs were highly motivated and that the programs were feasible for these cancer survivors.

Short-term and long-term comparisons. "Recovery \& Stability" and "OncoRev" belong to the few cancer rehabilitation programs worldwide that combined physical and psychosocial rehabilitation. The design of the "Recovery \& Stability" study did not offer the opportunity to compare physical and psychosocial interventions, to compare the effects of rehabilitation with no intervention, or to measure long-term effects. The "OncoRev" study tried to overcome some of these limitations. This latter study was the first to compare long-term effects on cancer survivors' quality of life of multi-modal rehabilitation versus physical training (chapters 4 and 5). Moreover, problem-solving and distress were also assessed, effects in subgroups of participants with higher versus lower pre-rehabilitation distress were investigated, as well as associations between (changes in) problemsolving and (changes in) distress (chapter 6). Strengths of the "OncoRev" study were the randomized controlled design for the comparison between the rehabilitation groups and the follow-up of participants up to 3 (chapter 4) and 9 (chapters 5 and 6) months after rehabilitation. Although the theorybased development of the physical as well as the cognitive-behavioral 
training as group-based self-management interventions was primarily considered a strength of the program, at the same time it might be perceived as a study limitation because it reduced the contrast between single focused and multi-modal rehabilitation. As randomized assignments of participants to no intervention were not feasible, a non-randomized comparison group of cancer survivors, waiting at least 3 months for rehabilitation in other centers in the Netherlands, was assessed at baseline and 3 months later (chapter 4). Longer follow-up assessments of this waiting-list comparison group were impossible, because cancer survivors usually do not have to wait for rehabilitation much longer than 3 months. A final limitation was that the separate effects of the cognitive-behavioral training in "OncoRev" were not measured. The reason was that cancer survivors who requested rehabilitation and knew that multi-modal rehabilitation was included in the design, would not want to take the risk to receive single focused rehabilitation.

\section{Generalizability}

The rehabilitation programs in this dissertation belong to the few cancer rehabilitation programs worldwide that focused on mixed groups of cancer survivors, i.e. that included other cancer survivors besides breast cancer survivors. Furthermore, the participants in our studies belonged to the approximately $26 \%$ of cancer survivors who express a need for rehabilitation (Van Harten et al. 1998). However, our results can not be generalized to all cancer survivors who might need cancer rehabilitation. Firstly, potential participants may not have entered rehabilitation, because they were not aware of the existence of cancer rehabilitation, or were not able to apply for cancer rehabilitation at their own initiative, or had difficulties to travel to the centers involved. Secondly, most participants were females and the majority of the participants had a breast cancer diagnosis, which implies that males and survivors of other cancer types were under represented. Furthermore, the programs more likely attract those who want to become physically active (chapter 3) and who should be regarded as a specific sample of the Dutch cancer population. Nevertheless, the programs were intended for those who opt for physical training. As the rehabilitation programs were conducted in six different centers throughout the Netherlands, the group of patients covered seems to be a good reflection of clinical and daily practice and the results of these studies may be representative for cancer survivors who usually apply for rehabilitation in the Netherlands. 


\section{Implications for practice}

This dissertation supports the notion that cancer rehabilitation after completion of medical treatment is a feasible approach, with substantial, durable and clinically relevant benefits, which can be broadly implemented and embedded in usual cancer care. In the past ten years, accessibility of cancer rehabilitation has grown in the Netherlands. Today, over 60 centers are offering cancer rehabilitation programs. Still, even more rehabilitation will be needed. It was estimated that, in the year 2000, 4,890 Dutch cancer survivors needed cancer rehabilitation and due to increasing incidence and survival rates this number is estimated to rise to 6,900 in 2015 (Gijsen et al. 2005). Offering cancer rehabilitation in more centers would also reduce the distance patients would have to travel for rehabilitation. Furthermore, it would enhance the awareness of available cancer rehabilitation in more cancer survivors, their health care professionals and others. The infrastructure of the "Recovery \& Stability Foundation" offers tools for implementing cancer rehabilitation at a larger scale and for continuous quality management through regular evaluations by participants and trainers, and educational and inter-vision activities for trainers. This foundation aims to increase accessibility of the program by promoting the idea that more health insurance companies cover the costs of cancer rehabilitation and that, moreover, these costs will be covered by the basic package of all health insurance companies. In the coming years Dutch cancer aftercare programs will be rigorously reorganized and standardized, based on a recent report of the Health Council of the Netherlands (2007) that emphasizes that health care professionals should systematically screen cancer patients during and after their primary medical treatment for their individual physical and psychosocial needs. Then, cancer survivors who are less able or reluctant to request rehabilitation by themselves might obtain easier access.

The greater benefits of the "OncoRev" program for quality of life compared with those of "Recovery \& Stability" indicate that in daily practice continuous quality monitoring, for instance by conducting focus groups, and updating the program is worthwhile. Furthermore, based on the "OncoRev" study (chapters 4, 5, and 6), one may consider that group-based physical training integrating a self-management approach sufficiently addresses the needs of cancer survivors. However, this might hold primarily for cancer survivors who are attracted to physical training. Due to the study-designs in this dissertation, one may not conclude that cognitive-behavioral training by itself would not have been beneficial for cancer survivors' quality of life. A 
cognitive-behavioral program might support, for instance, highly distressed cancer survivors who are not able or not motivated to enter physical training. However, given that quality of life is a multidimensional concept and also includes physical domains, promoting physical activity of the participants should then be one of the program goals.

The challenge is to keep on further fine tuning cancer rehabilitation to address individual participants' needs optimally and to promote costs and time effective rehabilitation. Variations on contents, duration, setting, and the timing of the program might be considered for specific subgroups. This fine tuning of cancer rehabilitation to the needs of individual participants may be facilitated by including more participants in rehabilitation, on the one hand, and by incorporating a self-management approach, on the other hand. Then, specific subgroups of participants might follow specific groupprograms, and within each group participants might work toward their individual goals. To date, the majority of participants in our rehabilitation studies were females with breast cancer. Women may have been more attracted than men to the psychosocial sessions and the group-format in rehabilitation because of collectivistic values, e.g. social interaction, whereas men might have preferred individual physical fitness training for reaching individual achievements. However, studies on gender differences in individualistic and collectivistic values have been inconsistent with one another (Ryckman 2003). Moreover, in a younger study population, although women assigned greater importance to collectivistic values than men, women and men did not differ in individualistic values (Ryckman 2003), and, in fact, women placed greater importance on achievement than men. In "OncoRev", cooperation within the group seemed helpful in achieving individual goals for men and women (May et al. 2007a). More men might enter rehabilitation if they were well informed about the value of a group program for reaching their individual goals, for instance by their health care professionals. Thus, efforts should be made to include more males and cancer survivors with other cancer types and to address their specific needs. The same holds for cancer survivors with different socio-economic and socio-cultural backgrounds. Homogeneous rehabilitation groups do not seem to be a requisite for effective rehabilitation as our studies showed that cancer rehabilitation was effective in mixed groups of cancer survivors.

The "OncoRev" study suggested that integrating self-management in physical training might be an effective strategy. This might imply that physical training interventions should change from therapist-oriented, i.e., the therapist prescribes the intervention while patients follow these instructions, to 
patient-oriented, where the role of the therapist shifts from healthcare provider to coach. To attain that goal, education of physical therapists should shift from a biomedical to a biopsychosocial perspective, incorporating selfmanagement issues, communication and coaching techniques.

\section{Implications for future research}

Future research might address several issues. Firstly, our studies did not compare cognitive-behavioral training with no intervention. Therefore, future research might compare usual care with the benefits of the groupbased cognitive-behavioral problem-solving training for Dutch cancer survivors' quality of life. The large and durable effects of problem-solving training on quality of life in distressed cancer patients in the United States in particular (Nezu et al. 2003), and of cognitive-behavioral interventions in cancer populations in general (Osborn et al. 2006) make such an investigation relevant. In the Netherlands individual cognitive-behavioral therapy, especially designed to reduce fatigue in highly fatigued cancer survivors, had long-term positive effects on their fatigue, functional impairments and psychological distress (Gielissen et al. 2007). Presently, a trial is being prepared in several Dutch radio-therapeutic institutes to compare usual care with individual cognitive-behavioral training for highly distressed cancer survivors after radio-therapy, based on the protocol of Nezu et al. (1998) that was also used in the "OncoRev" cognitive-behavioral training module (www.kankerbestrijding.nl). However, from the perspective of costs effectiveness and because group interventions were reported to be at least as effective as individual interventions in cancer patients (Sheard and Maguire 1999), it might also be worthwhile to compare usual care with group-based cognitive-behavioral training in Dutch cancer survivors. Secondly, integrating the self-management approach in the physical training in "OncoRev" seemed to be an effective approach. Nonetheless, the most rigorous evaluation of the value of the self-management approach in physical training in cancer rehabilitation would be a randomized study comparing physical training not based on self-management with physical training based on a self-management approach. Thirdly, the potential benefits of a group-wise approach beyond those of an individual approach might be tested in a randomized trial comparing individual self-management physical training with group-based self-management physical training. Fourthly, future research might also focus on the costs and time effectiveness of cancer rehabilitation. A recent study showed that long-term cancer survivors visit 
their medical specialists more often than the general Dutch population (Mols et al. 2007). Therefore, it is useful to investigate the costs of cancer rehabilitation in relation to its potential to reduce the costs of, for instance, health care utilization and days of sick leave. Lastly, research might be aimed at enhancing accessibility of cancer rehabilitation for more cancer survivors. For example, studies might focus on how to reach more male cancer survivors, more cancer survivors with other cancer types than breast cancer, and different socio-economic subgroups.

\section{General conclusion}

To conclude, comprehensive group-based physical training based on selfmanagement principles had substantial and durable positive effects on quality of life of cancer survivors who experience physical and/or psychosocial problems after their completion of primary medical treatment. Combining this self-management training with cognitive-behavioral training did not show additional benefits, neither in the short-term nor in the long-term. Therefore, group-based self-management physical training is sufficient for most cancer survivors who apply for rehabilitation that includes physical training, and can be embedded in usual cancer care. 



\section{References}

Aaronson, N. K., Ahmedzai, S., Bergman, B., Bullinger, M., Cull, A., Duez, N. J., Filiberti, A., Flechtner, H., Fleishman, S. B., De Haes, J. C., \& et al. (1993). The European Organization for Research and Treatment of Cancer QLQ-C30: a quality-of-life instrument for use in international clinical trials in oncology. Journal of the National Cancer Institute, 85, 365-76.

Andersen, B. L. (2002). Biobehavioral outcomes following psychological interventions for cancer patients. Journal of Consulting and Clinical Psychology, 70, 590-610.

Antoni, M. H., Lechner, S. C., Kazi, A., Wimberly, S. R., Sifre, T., Urcuyo, K. R., Phillips, K., Gluck, S., \& Carver, C. S. (2006). How stress management improves quality of life after treatment for breast cancer. Journal of Consulting and Clinical Psychology, 74, 1143-52.

Apolone, G., Filiberti, A., Cifani, S., Ruggiata, R., \& Mosconi, P. (1998). Evaluation of the EORTC QLQ-C30 questionnaire: a comparison with SF-36 Health Survey in a cohort of Italian long-survival cancer patients. Annals of Oncology, 9, 549-57.

Bandura, A. (1986). Social foundations of thought and action: a social cognitive theory. Englewood Cliffs: Prentice-Hall.

Bandura, A. (1997). Self-efficacy: the exercise of control. New York: Freeman.

Barrett, B., Brown, D., Mundt, M., \& Brown, R. (2005). Sufficiently important difference: expanding the framework of clinical significance. Medical Decision Making, 25, 25061.

Berglund, G., Bolund, C., Gustafsson, U. L., \& Sjoden, P. O. (1994). One-year follow-up of the 'Starting Again' group rehabilitation programme for cancer patients. European Journal of Cancer, 30, 1744-51.

Berglund, G., Bolund, C., Gustafsson, U. L., \& Sjoden, P. O. (1997). Is the wish to participate in a cancer rehabilitation program an indicator of the need? Comparisons of participants and non-participants in a randomized study. Psycho-Oncology, 6, 35-46.

Bjordal, K., Mastekaasa, A., \& Kaasa, S. (1995). Self-reported satisfaction with life and physical health in long-term cancer survivors and a matched control group. European Journal of Cancer, 31, 340-5. 
Blackwell, E., de Leon, C. F., \& Miller, G. E. (2006). Applying mixed regression models to the analysis of repeated-measures data in psychosomatic medicine. Psychosomatic Medicine, 68, 870-8.

Bogart, L. M., \& Helgeson, V. S. (2000). Social comparisons among women with breast cancer: a longitudinal investigation. Journal of Applied Psychology, 30, 547-575.

Borg, G. A. (1982). Psychophysical bases of perceived exertion. Medicine and Science in Sports and Exercise, 14, 377-81.

Bower, J. E., Ganz, P. A., Desmond, K. A., Bernaards, C., Rowland, J. H., Meyerowitz, B. E., $\&$ Belin, T. R. (2006). Fatigue in long-term breast carcinoma survivors: a longitudinal investigation. Cancer, 106, 751-8.

Chang, E. C., D'Zurilla, T. J., \& Sanna, L. J. (2004). Social problem-solving: theory, research and training. Washington: American Psychological Association.

Cohen, J. (1988). Statistical power analysis for the behavioral sciences. New York: Academic Press.

Conn, V. S., Hafdahl, A. R., Porock, D. C., McDaniel, R., \& Nielsen, P. J. (2006). A metaanalysis of exercise interventions among people treated for cancer. Supportive Care in Cancer, 14, 699-712.

Courneya, K. S., \& Friedenreich, C. M. (1999). Physical exercise and quality of life following cancer diagnosis: a literature review. Annals of Behavioral Medicine, 21, 171-9.

Courneya, K. S., Friedenreich, C. M., Sela, R. A., Quinney, H. A., Rhodes, R. E., \& Handman, M. (2003a). The group psychotherapy and home-based physical exercise (grouphope) trial in cancer survivors: physical fitness and quality of life outcomes. PsychoOncology, 12, 357-74.

Courneya, K. S., Mackey, J. R., Bell, G. J., Jones, L. W., Field, C. J., \& Fairey, A. S. (2003b). Randomized controlled trail of exercise training in postmenopausal breast cancer survivors: cardiopulmonary and quality of life outcomes. Journal of Clinical Oncology, 21, 1660-8.

Creer, T. L. (2000). Self-management of chronic illness. In M. Boekaerts, P. R. Pintrich \& M. Zeidner (Eds.), (2000). Handbook of self-regulation. (pp. 601-29). San Diego: Academic Press.

Curt, G. A., Breitbart, W., Cella, D., Groopman, J. E., Horning, S. J., Itri, L. M., Johnson, D. H., Miaskowski, C., Scherr, S. L., Portenoy, R. K., \& Vogelzang, N. J. (2000). Impact of cancer-related fatigue on the lives of patients: new findings from the Fatigue Coalition. The Oncologist, 5, 353-60.

D'Zurilla, T. J., \& Goldfried, M. R. (1971). Problem-solving and behavior modification. Journal of Abnormal Psychology, 78, 107-26.

D'Zurilla, T. J., \& Nezu, A. M. (2007). Problem-solving therapy: a positive approach to clinical intervention. New York: Springer.

D'Zurilla, T. J., Nezu, A. M., \& Maydeu-Olivares, A. (2002). Social Problem-Solving Inventory - Revised (SPSI-R). North Tonawanda: Multi-Health Systems.

Daley, A. J., Crank, H., Saxton, J. M., Mutrie, N., Coleman, R., \& Roalfe, A. (2007). Randomized trial of exercise therapy in women treated for breast cancer. Journal of Clinical Oncology 25, 1713-21.

De Haes, J. C. J. M. (1988). Kwaliteit van leven van kankerpatiënten, Thesis Leiden University.

Elbl, L., Vasova, I., Tomaskova, I., Jedlicka, F., Navratil, M., Pospisil, Z., \& Vorlicek, J. (2006). Cardiac function and cardiopulmonary performance in patients after treatment for non-Hodgkin's lymphoma. Neoplasma, 53, 174-81. 
Engel, G. L. (1977). The need for a new medical model: A challenge for biomedicine. Science, 196, 129-36.

Engel, G.L. (1980). The clinical application of the biopsychosocial model. American Journal of Psychiatry, 137, 535-44.

Engel, G.L. (1997). From biomedical to biopsychosocial. Being scientific in the human domain. Psychosomatics, 38, 521-8.

EORTC (2003). The EORTC QLQ-C30 Manuals, Reference Values and Bibliography. In Quality of Life Group \& Quality of Life Unit (Eds.). Brussels: EORTC Data Center Quality of Life Unit.

Fawzy, F. I., Fawzy, N. W., Arndt, L. A., \& Pasnau, R. O. (1995). Critical review of psychosocial interventions in cancer care. Archives of General Psychiatry, 52, 100-13.

Festinger, L. (1954). A theory of social comparison processes. Human Relations, 7, 117-40.

Ganz, P. A., Coscarelli, A., Fred, C., Kahn, B., Polinsky, M. L., \& Petersen, L. (1996). Breast cancer survivors: psychosocial concerns and quality of life. Breast Cancer Research and Treatment, 38, 183-99.

Gielissen, M. F., Verhagen, C. A., \& Bleijenberg, G. (2007). Cognitive behaviour therapy for fatigued cancer survivors: long-term follow-up. British Journal of Cancer, 97, 612-8.

Gijsen, B. C. M., Hellendoorn-van Vreeswijk, A. J. H., Koppejan-Rensenbrink, A. G., \& Remie, M. E. (2005). Kanker en Revalidatie: Herstel en Balans, een innovatief programma. Utrecht: Vereniging van Integrale Kankercentra.

Gotay, C. C., Holup, J. L., \& Pagano, I. (2002). Ethnic differences in quality of life among early breast and prostate cancer survivors. Psycho-Oncology, 11, 103-13.

Goubert, L., Crombez, G., Van Damme, S., Vlaeyen, J. W. S., Bijttebier, P., \& Roelofs, J. (2004). Confirmatory Factor Analysis of the Tampa Scale for Kinesiophobia: Invariant Two-Factor Model Across Low Back Pain Patients and Fibromyalgia Patients. Clinical Journal of Pain, 20, 103-10.

Graves, K. D. (2003). Social cognitive theory and cancer patients' quality of life: a metaanalysis of psychosocial intervention components. Health Psychology, 22, 210-9.

Hagger, M. S., \& Orbell, S. (2003). A meta-analytic review of the common-sense model of illness representations. Psychology and Health, 18, 141-84.

Health Council of the Netherlands (2007). Follow-up in oncology. Identify objectives, substantiate actions (pp. 1-154 publication no. 20007/10). The Hague.

Helgeson, V. S., \& Cohen, S. (1996). Social support and adjustment to cancer: Reconciling descriptive, correlational, and intervention research. Health Psychology, 15, 135-48.

Helgeson, V. S., Cohen, S., Schulz, R., \& Yasko, J. (2001). Long-term effects of educational and peer discussion group interventions on adjustment to breast cancer. Health Psychology, 20, 387-92.

Holroyd, K. A., \& Creer, T. L. (1986). Self-management of chronic disease: handbook of clinical interventions and research. Orlando: Academic Press.

Irwin, M. L., \& Ainsworth, B. E. (2004). Physical activity interventions following cancer diagnosis: methodologic challenges to delivery and assessment. Cancer Investigation, $22,30-50$.

Karvinen, K. H., Courneya, K. S., North, S., \& Venner, P. (2007). Associations between exercise and quality of life in bladder cancer survivors: a population-based study. Cancer Epidemiology, Biomarkers and Prevention, 16, 984-90.

Karvonen, J., \& Vuorimaa, T. (1988). Heart rate and exercise intensity during sports activities. Practical application. Sports Medicine, 5, 303-11. 
King, M. T. (1996). The interpretation of scores from the EORTC quality of life questionnaire QLQ-C30. Quality of Life Research, 5, 555-67.

Kole-Snijders, A. J. M., Geurts, S. M., Van den Hout, J. H. C., \& Vlaeyen, J. W. S. (2006). Probleemoplossende vaardigheidstraining: een groeps- en individueel programma. Amsterdam: Boom.

Korstjens, I., May, A. M., Van Weert, E., Mesters, I., Tan, F., Ros, W. J. G., HoekstraWeebers, J. E. H. M., Van der Schans, C. P., \& Van den Borne, B. (2008a). Quality of life after self-management cancer rehabilitation: a randomized controlled trial comparing physical and cognitive-behavioral training versus physical training. Psychosomatic Medicine, 70, 422-9. DOI:10.1097/PSY.0b013e31816e038f.

Korstjens, I., May, A. M., Van Weert, E., Mesters, I., \& Van den Borne, B. (2006a). Quality of Life after multidisciplinary cancer rehabilitation, combining physical training and psycho-eduction. Psycho-Oncology, 15 (S2), S293.

Korstjens, I., Mesters, I., Gijsen, B. C. M., \& Van den Borne, B. (2008b). Cancer patients' view on rehabilitation and quality of life: a programme audit. European Journal of Cancer Care, 17, 290-7. DOI: 10.1111/j.1365-2354.2007.00864.x.

Korstjens, I., Mesters, I., Van der Peet, E., Gijsen, B., \& Van den Borne, B. (2006b). Quality of life of cancer survivors after physical and psychosocial rehabilitation. European Journal of Cancer Prevention, 15, 541-7.

Krarup, L.H., Gluud, C., Truelsen, T., Pedersen, A., Lindahl, M., Hansen, L., Michelsen, S., Andersen, G., Zeng, X., Korv, J., Oskedra, A., \& Boysen, G. (2008). The ExStroke Pilot Trial: rationale, design, and baseline data of a randomized multicenter trial comparing physical training versus usual care after an ischemic stroke. Contempory Clinical Trials, DOI: 10.1016/j.cct.2007.09.008.

Lazarus, R. S. (1993). Coping theory and research: past, present, and future. Psychosomatic Medicine, 55, 234-47.

Leventhal, H., \& Carr, S. (2001). Speculations on the relationship of behavioral therapy to psychosocial research on cancer. In A. Baum \& B. L. Andersen (Eds.), Psychosocial interventions for cancer (pp. p. 375-400). Washington: American Psychological Association.

Maas, C. J. M., \& Hox, J. J. (2005). Sufficient sample sizes for multilevel modeling. Methodology, 1, 85-91.

Maddux, J. E. (1995). Self-efficacy theory: an introduction. In J. E. Maddux (Ed.), Selfefficacy, adaptation, and adjustment: theory, research, and application. (pp. 3-33). New York: Plenum Press.

May, A. M., Duivenvoorden, H. J., Korstjens, I., Van Weert, E., Hoekstra-Weebers, J. E. H. M., Van den Borne, B., Mesters, I., Van der Schans, C. P., \& Ros, W. J. G. (2007a). The effect of group cohesion on rehabilitation outcome in cancer survivors. PsychoOncology, DOI: 10.1002/pon.1308.

May, A. M., Korstjens, I., Van Weert, E., Van den Borne, B., Hoekstra-Weebers, J. E. H. M., Van der Schans, C. P., Mesters, I., Passchier, J., Grobbee, D. E., \& Ros, W. J. G. (2007b). Long-term effects on cancer survivors' quality of life of physical training versus physical training combined with cognitive-behavioral therapy: a randomized clinical trial. In A. M. May (Ed.), Rehabilitation in cancer: training and talking? Utrecht: Thesis Utrecht University. 
May, A. M., Van Weert, E., Korstjens, I., Hoekstra-Weebers, J. E. H. M., van der Schans, C. P., Zonderland, M. L., Mesters, I., Van den Borne, B., \& Ros, W. J. G. (2008). Improved physical fitness of cancer survivors: a randomized controlled trial comparing physical training with physical and cognitive-behavioural training. Acta Oncologica, 47, 825-34. DOI: 10.1080/02841860701666063.

McLachlan, S. A., Devins, G. M., \& Goodwin, P. J. (1998). Validation of the European Organization for Research and Treatment of Cancer Quality of Life Questionnaire (QLQ-C30) as a measure of psychosocial function in breast cancer patients. European Journal of Cancer, 34, 510-7.

McNeely, M. L., Campbell, K. L., Rowe, B. H., Klassen, T. P., Mackey, J. R., \& Courneya, K. S. (2006). Effects of exercise on breast cancer patients and survivors: a systematic review and meta-analysis. Canadian Medical Association Journal, 175, 34-41.

Mesters, I., Creer, T. L., \& Gerards, F. (2002). Self-management and respiratory disorders: guiding from health counseling and self-management perspectives. In A. Kaptein \& $\mathrm{T}$. L. Creer (Eds.), Respiratory disorders and behavioral research (pp. 139-74). London: Dunitz.

Meyer, T. J., \& Mark, M. M. (1995). Effects of psychosocial interventions with adult cancer patients: a meta-analysis of randomized experiments. Health Psychology, 14, 101-8.

Michael, Y. L., Kawachi, I., Berkman, L. F., Holmes, M. D., \& Colditz, G. A. (2000). The persistent impact of breast carcinoma on functional health status: prospective evidence from the Nurses' Health Study. Cancer, 89, 2176-86.

Milne, H. M., Wallman, K. E., Gordon, S., \& Courneya, K. S. (2007). Effects of a combined aerobic and resistance exercise program in breast cancer survivors: a randomized controlled trial. Breast Cancer Research and Treatment, DOI: 10.1007/s10549-007-9602-z.

Mock, V., Burke, M. B., Sheehan, P., Creaton, E. M., Winningham, M. L., McKenney Tedder, S., Schwager, L. P., \& Liebman, M. (1994). A nursing rehabilitation program for women with breast cancer receiving adjuvant chemotherapy. Oncology Nursing Forum, 21, 899-907.

Mols, F., Helfenrath, K. A., Vingerhoets, A. J., Coebergh, J. W., \& van de Poll Franse, L. V. (2007). Increased health care utilization among long-term cancer survivors compared to the average Dutch population: a population-based study. International Journal of Cancer, 121, 871-7.

Morgan, D. L., \& Krueger, R. A. (1998). The focus group kit. Thousand Oaks: Sage.

Nezu, A. M. (2004). Problem-solving and behavior therapy revisited. Behavior Therapy, 35, $1-33$.

Nezu, A. M., Nezu, C. M., Felgoise, S. H., McClure, K. S., \& Houts, P. S. (2003). Project Genesis: assessing the efficacy of problem-solving therapy for distressed adult cancer patients. Journal of Consulting and Clinical Psychology, 71, 1036-48.

Nezu, A. M., Nezu, C. M., Friedman, S. H., Faddis, S., \& Houts, P. S. (1998). Helping cancer patients cope: a problem-solving approach. Washington: American Psychological Association.

Nezu, A. M., Nezu, C. M., Houts, P. S., Friedman, S. H., \& Faddis, S. (1999a). Relevance of problem-solving therapy to psychosocial oncology. Journal of Psychosocial Oncology, $16,5-26$.

Nezu, C. M., Nezu, A. M., Friedman, S. H., Houts, P. S., DelliCarpini, L., Bildner, C., \& Faddis, S. (1999b). Cancer and psychological distress: two investigations regarding the role of social problem-solving. Journal of Psychosocial Oncology, 16, 27-40. 
Nunnally, J. C. (1970). Introduction to psychological measurement. New York: Mc GrawHill.

Nunnally, J.C. (1978). Psychometric Theory. New York: McGraw-Hill.

Oldervoll, L. M., Kaasa, S., Hjermstad, M. J., Lund, J. A., \& Loge, J. H. (2004). Physical exercise results in the improved subjective well-being of a few or is effective rehabilitation for all cancer patients? European Journal of Cancer, 40, 951-62.

Osborn, R. L., Demoncada, A. C., \& Feuerstein, M. (2006). Psychosocial interventions for depression, anxiety, and quality of life in cancer survivors: meta-analyses. International Journal of Psychiatry in Medicine, 36, 13-34.

Osoba, D., Rodrigues, G., Myles, J., Zee, B., \& Pater, J. (1998). Interpreting the significance of changes in health-related quality-of-life scores. Journal of Clinical Oncology, 16, 139-44.

Parkin, D. M., Bray, F. I., \& Devesa, S. S. (2001). Cancer burden in the year 2000. The global picture. European Journal of Cancer, 37 Suppl 8, S4-66.

Pennebaker, J. W., \& Beall, S. K. (1986). Confronting a traumatic event: toward an understanding of inhibition and disease. Journal of Abnormal Psychology, 95, 274-81.

Rehse, B., \& Pukrop, R. (2003). Effects of psychosocial interventions on quality of life in adult cancer patients: meta analysis of 37 published controlled outcome studies. Patient Education and Counseling, 50, 179-86.

Rummans, T. A., Clark, M. M., Sloan, J. A., Frost, M. H., Bostwick, J. M., Atherton, P. J., Johnson, M. E., Gamble, G., Richardson, J., Brown, P., Martensen, J., Miller, J., Piderman, K., Huschka, M., Girardi, J., \& Hanson, J. (2006). Impacting quality of life for patients with advanced cancer with a structured multidisciplinary intervention: a randomized controlled trial. Journal of Clinical Oncology, 24, 635-42.

Ryckman, R.M. \& Houston, D.M. (2003). Value priorities in American and British female and male university students. The Journal of Social Psychology, 143, 127-38.

Scheier, M. F., Helgeson, V. S., Schulz, R., Colvin, S., Berga, S., Bridges, M. W., Knapp, J., Gerszten, K., \& Pappert, W. S. (2005). Interventions to enhance physical and psychological functioning among younger women who are ending nonhormonal adjuvant treatment for early-stage breast cancer. Journal of Clinical Oncology, 23, 4298-311.

Schmitz, K. H., Holtzman, J., Courneya, K. S., Masse, L. C., Duval, S., \& Kane, R. (2005). Controlled physical activity trials in cancer survivors: a systematic review and metaanalysis. Cancer Epidemiology, Biomarkers and Prevention, 14, 1588-95.

Schuit, A. J., Schouten, E. G., Westerterp, K. R., \& Saris, W. H. (1997). Validity of the Physical Activity Scale for the Elderly (PASE): according to energy expenditure assessed by the doubly labeled water method. Journal of Clinical Epidemiology, 50, 5416.

Schwartz, A. L. (2004). Physical activity after a cancer diagnosis: psychosocial outcomes. Cancer Investigation, 22, 82-92.

Segar, M. L., Katch, V. L., Roth, R. S., Garcia, A. W., Portner, T. I., Glickman, S. G., Haslanger, S., \& Wilkins, E. G. (1998). The effect of aerobic exercise on self-esteem and depressive and anxiety symptoms among breast cancer survivors. Oncology Nursing Forum, 25, 107-13.

Servaes, P., Verhagen, C., \& Bleijenberg, G. (2002). Fatigue in cancer patients during and after treatment: prevalence, correlates and interventions. European Journal of Cancer $38,27-43$. 
Sheard, T., \& Maguire, P. (1999). The effect of psychological interventions on anxiety and depression in cancer patients: results of two meta-analyses. British Journal of Cancer, 80, 1770-80.

Spinhoven, P., Ormel, J., Sloekers, P. P. A., Kempen, G. I. J. M., Speckens, A. E. M., \& Van Hemert, A. M. (1997). A validation study of the Hospital Anxiety and Depression Scale (HADS) in different groups of Dutch subjects. Psychological Medicine, 27, 363-70.

SPSS (2002). SPSS for Windows, Release 11.5. Chicago: SPSS.

SPSS (2004). SPSS for Windows, Release 13.0. Chicago: SPSS.

Stanton, A. L., Danoff Burg, S., Cameron, C. L., Snider, P. R., \& Kirk, S. B. (1999). Social comparison and adjustment to breast cancer: an experimental examination of upward affiliation and downward evaluation. Health Psychology, 18, 151-8.

Stull, V. B., Snyder, D. C., \& Demark Wahnefried, W. (2007). Lifestyle interventions in cancer survivors: designing programs that meet the needs of this vulnerable and growing population. Journal of Nutrition, 137, 243s-248s.

Sweeney, C., Schmitz, K. H., Lazovich, D., Virnig, B. A., Wallace, R. B., \& Folsom, A. R. (2006). Functional limitations in elderly female cancer survivors. Journal of the National Cancer Institute, 98, 521-9.

Taylor, S. E., \& Brown, J. D. (1988). Illusion and well-being: a social psychological perspective on mental health. Psychological Bulletin, 103, 193-210.

Taylor, S. E., \& Lobel, M. (1989). Social comparison activity under threat: downward evaluation and upward contacts. Psychological Review, 96, 569-575.

Tobin, T. L., Reynolds, R. V. C., Holroyd, K. A., \& Creer, T. L. (1986). Self-management and social learning theory. In K. A. Holroyd \& T. L. Creer (Eds.), Self-management of chronic disease: handbook of clinical interventions and research (pp. 29-55). Orlando: Academic Press.

Tomich, P. L., \& Helgeson, V. S. (2002). Five years later: a cross-sectional comparison of breast cancer survivors with healthy women. Psycho-Oncology, 11, 154-69.

Van den Hout, J. H. C., Vlaeyen, J. W., Heuts, P. H. T. G., Sillen, W. J. T., \& Willen, J. E. H. L. (2001). Functional disability in non-specific low back pain: the role of pain-related fear and problem-solving skills. International Journal of Behavioral Medicine, 8, 134-48.

Van den Hout, J. H. C., Vlaeyen, J. W. S., Heuts, P. H. T. G., Zijlema, J. H. L., \& Wijnen, J. A. G. (2003). Secondary prevention of work-related disability in nonspecific low back pain: Does problem-solving therapy help? A randomized clinical trial. Clinical Journal of Pain, 19, 87-96.

Van der Zee, K. I., \& Sanderman, R. (1993). Measuring general health status with the RAND36. User Manual. Groningen: Northern Center of Health Care Research.

Van der Zee, K. I., Sanderman, R., Heyink, J. W., \& De Haes, H. (1996). Psychometric qualities of the RAND 36-Item Health Survey 1.0: a multidimensional measure of general health status. International Journal of Behavioral Medicine, 3, 104-22.

Van Harten, W. H., Van Noort, O., Warmerdam, R., Hendricks, H., \& Seidel, E. (1998). Assessment of rehabilitation needs in cancer patients. International Journal of Rehabilitation Research, 21, 247-57.

Van Hemert, A. M., \& Ormel, J. (1996). Nederlandse versie van de Hospital Anxiety and Depression Scale (HADS). Groningen: Rijksuniversiteit Groningen. 
Van Weert, E., Hoekstra-Weebers, J., Grol, B., Otter, R., Arendzen, H. J., Postema, K., Sanderman, R., \& Van der Schans, C. (2005). A multidimensional cancer rehabilitation program for cancer survivors: effectiveness on health-related quality of life. Journal of Psychosomatic Research, 58, 485-96.

Van Weert, E., Hoekstra-Weebers, J. E. H. M., Grol, B. M. F., Otter, R., Arendzen, J. H., Postema, K., \& Van der Schans, C. P. (2004). Physical functioning and quality of life after cancer rehabilitation. International Journal of Rehabilitation Research, 27, 27-35.

Van Weert, E., Hoekstra Weebers, J. E. H. M., May, A. M., Korstjens, I., Ros, W. J. G., \& Van der Schans, C. P. (2008). The development of an evidence-based physical selfmanagement rehabilitation programme for cancer survivors. Patient Education and Counseling 71, 169-90. DOI: 10.1016/j.pec.2007.11.027.

Vlaeyen, J. W., Smeets, R. J., Mulder, H. P., Pelt, R. A., \& Bakker Boerrigter, A. H. (1993). Secundaire preventie van chronische lage rugpijn: een gedragsgeoriënteerde benadering. Nederlands Tijdschrift voor Geneeskunde, 137, 1544-9.

Walters, S. J., \& Brazier, J. E. (2003). What is the relationship between the minimally important difference and health state utility values? The case of the SF-6D. Health and Quality of Life Outcomes, 1, 1-8.

Ware, J. E., \& Sherbourne, C. D. (1992). The MOS 36-item short-form health survey (SF-36):

I. Conceptual framework and item selection. Medical Care, 30, 473-83

www.ikcnet.nl. Cited April 2008.

www.kankerbestrijding.nl Project RUG 2007-3805. Cited April 2008.

www.who.int. Cited April 2008

Zigmond, A. S., \& Snaith, R. P. (1983). The Hospital Anxiety and Depression Scale. Acta Psychiatrica Scandinavia, 67, 361-70.

Zimmerman, B. J. (2000). Attaining self-regulation: a social cognitive perspective. In M. Boekaerts, P. R. Pintrich \& M. Zeidner (Eds.), (2000). Handbook of self-regulation (pp. 13-39). San Diego, USA: Academic Press. 
Summary \& Samenvatting 



\section{Summary}

Because cancer incidence as well as survival rates of persons with cancer are growing, more and more persons have to manage their cancer as a chronic disease. About $26 \%$ of cancer survivors report decreased quality of life and express a need for professional support in managing physical, psychological and social problems. The number of Dutch cancer survivors needing rehabilitation is expected to rise from 4,890 in the year 2000 to at least 6,900 in 2015. This dissertation addresses the development and evaluation of rehabilitation programs for cancer survivors to improve their quality of life. The main aims were, first, to investigate the effects of multi-modal physical and psychosocial rehabilitation on cancer survivors' quality of life and, second, to investigate whether multi-modal physical and psychosocial rehabilitation was more effective than single-focused physical rehabilitation. First, the foundation and evaluation of "Recovery \& Stability" ("Herstel \& Balans"), an already since 1996 existing Dutch multi-modal cancer rehabilitation program is described (chapters 2 and 3). Second, the development and evaluation of "OncoRev", a recently developed cancer rehabilitation program (chapters 4, 5, and 6) is presented. In the study reported in these chapters, multi-modal rehabilitation was compared with single-focused rehabilitation. The participants in both rehabilitation programs were cancer survivors (all cancer types) who had successfully completed their primary cancer treatment at least 2 ("Recovery \& Stability") or 3 ("OncoRev") months ago, had a life expectancy of at least 1 year, but were still struggling with physical, psychological, and/or social problems.

\section{The rehabilitation programs}

The Dutch 12-week group-based rehabilitation program "Recovery \& Stability" consisted of physical training sessions (twice weekly), guided by physiotherapists, and 7 psychosocial sessions, guided by different professionals addressing different themes. Because the developers of "Recovery \& Stability" acknowledged the biopsychosocial model and the theory on social comparison, social support and modeling, this multi-modal program aimed to improve the physical, psychological, and social domains of cancer survi- 
vors' quality of life. Physical training was aimed at improving movement skills, strength and endurance, coping with physical complaints like fatigue, enhancing feelings of control and stress reduction. The physical graded activity training started at a low level and was gradually build up, following an individualized training scheme. Each physical training session consisted of individual training of strength and endurance or a group sports and games program, aqua aerobics in an indoor swimming pool, and a break for mutual contact and sharing experiences among the participants. The psychosocial sessions aimed at providing support in coping with cancer and enhancing self-confidence and autonomy. The participants were provided with information on cancer-related subjects and were given opportunities to share their experiences as cancer survivors. The program was implemented in 1996 in 2 rehabilitation centers. To date, cancer rehabilitation based on this multimodal program is being offered in more than 60 centers throughout the Netherlands and Belgium and is becoming part of regular care.

Based on the studies concerning "Recovery \& Stability", the "OncoRev" project group (page 4) developed a new program. In 2004, in 4 Dutch centers, the 12-week group-based rehabilitation program "OncoRev" started. The physical training sessions (twice weekly) as well as psychosocial training sessions (once weekly) in this program were based on standardized self-management protocols and guided by therapists. In this program, the multi-modal and group-wise approach was preserved, and, additionally, other social cognitive concepts, e.g. self-efficacy, self-management, and problem-solving theory were integrated in the program components. The circular self-management process comprises: 1) goal selection, 2) information collection, 3) information processing and evaluation, 4) decision making, 5) action and self-reaction. Physical training consisted of a personalized exercise program based on baseline exhaustive exercise testing and on goals set by the participants in cooperation with their therapists. Each session consisted of individual exercise followed by group sports. During the physical training, the participants used heart rate monitors, a scale measuring dyspnoea and fatigue, and training logs to monitor and evaluate their performance. They received feedback, information and support from their therapists in regulating their performance. During the psychosocial training sessions and in home-work assignments, the participants learned to apply self-management skills in striving for personal goals (e.g. in physical activity, work, household, hobbies, family relations and social contacts). The first 3 weeks focused primarily on exchanging participants' experiences with 
cancer, psycho-education about stress, relaxation, fatigue, exercise physiology, illness perceptions, as well as on promoting optimism and self-efficacy for self-management. Then, participants were primarily trained in practicing the steps in the circular problem-solving process: 1) problem orientation; 2) problem definition and formulation, and goal setting; 3) generation of alternative solutions (brainstorming); 4) decision-making; and 5) solution implementation and verification.

\section{The studies}

A longitudinal cohort study $(1996-2002 ; \mathrm{n}=658)$ investigated the effects of the "Recovery \& Stability" program on cancer survivors' quality of life (chapter 2). Most participants (78\%) were women, and half of the participants $(54 \%)$ had a breast cancer diagnosis. The mean age was 51 years and on average participants entered rehabilitation 1.3 years after they completed medical treatment. The low dropout of participants (4\% halfway and 13\% after twelve weeks) indicated that the program was feasible and that the participants were highly motivated. Halfway rehabilitation significant improvements were found in all domains of quality of life, except in cognitive functioning. After rehabilitation, cancer survivors reported significant improvements in all domains of quality of life. However, these improvements were clinically relevant only for 2 subgroups of participants, i.e. for non-working participants in role functioning, and for participants with other cancer diagnoses than breast cancer in physical functioning and social functioning. The "Recovery \& Stability" study had a large sample size and showed positive effects of rehabilitation in mixed groups of cancer survivors (all cancer types). Next, a focus group study explored the view of former participants in "Recovery \& Stability" on how and to what extent the program had met their needs (chapter 3). This study described their experiences during and after the program, their views on the value of cancer rehabilitation and their suggestions for improvement of the program. The focus group participants represented the longitudinal study population in baseline characteristics. They valued rehabilitation as an important stepping stone in their ongoing recovery process. Furthermore, they saw the combination of physical and psychosocial sessions and the group-format as strengths of the "Recovery \& Stability" program, and they suggested that the program should focus more on developing skills to cope with cancer. 
The studies on "Recovery \& Stability" (chapters 2 and 3) suggested that multi-modal rehabilitation was feasible and effective in mixed groups of cancer survivors, but that there was room for improvement of the program. The "OncoRev" project group also considered that a more rigorous evaluation of the separate modules and a comparison with no intervention was needed. We hypothesized that integrating cognitive-behavioral elements into physical training might enhance the effects of physical training on quality of life and that combining such a comprehensive physical training with a cognitive-behavioral psychosocial training could lead to even greater improvements in cancer survivors' quality of life.

The randomized controlled "OncoRev" trial (2004-2007) investigated the effects of single-focused rehabilitation $(\mathrm{n}=71)$ and multi-modal rehabilitation $(\mathrm{n}=76)$ on cancer survivors' quality of life, problem-solving and distress, immediately after rehabilitation and at 3 and 9 months after rehabilitation (chapters 4, 5, and 6). Single focused rehabilitation consisted of comprehensive self-management physical training and multi-modal rehabilitation contained comprehensive self-management physical training as well as self-management cognitive-behavioral training. Additionally, the effects of both rehabilitation groups were compared with a waiting-list comparison group $(\mathrm{n}=61)$ immediately after rehabilitation (chapter 4$)$.

Most participants (86\%) were women, and over half of the participants (57\%) had a breast cancer diagnosis (chapter 4). The mean age was 50 years and on average participants entered rehabilitation 1.5 years after they completed medical treatment. Dropout of participants was low; $8 \%$ of the participants in rehabilitation immediately after rehabilitation, $10 \%$ at 3month follow-up and $17 \%$ at 9 -month follow-up (chapter 5). Immediately after rehabilitation, participants in the rehabilitation groups showed significant clinically relevant improvements, predominantly in physical domains of quality of life, i.e. reduced role limitations due to physical problems, better physical functioning, and improved vitality and health change as compared with waiting-list participants with no intervention (chapter 4). The effects on cancer survivors' quality of life of multi-modal rehabilitation did not significantly differ from those of single focused rehabilitation at any of the followup measurements (chapters 4 and 5). Compared with pre-rehabilitation, participants in the rehabilitation groups reported significant improvements in all domains of quality of life at all follow-up measurements (chapters 4 and 5). These improvements remained clinically relevant in almost all domains of quality of life at 9 months after rehabilitation (chapter 5). 
Compared with pre-rehabilitation, participants in the rehabilitation groups also showed significant reductions in one domain of problem-solving, i.e. negative problem orientation, and in distress, i.e. anxiety and depression, at all follow-up measurements (chapter 6). Furthermore, also in problemsolving and distress multi-modal rehabilitation did not outperform single focused rehabilitation. Subsequent detailed analysis of the effects of the interventions on participants with higher levels of pre-rehabilitation distress compared with participants with lower pre-rehabilitation distress showed no difference in benefits in problem solving and distress between the 2 interventions at any of the follow-up measurements (chapter 6). Reduced negative problem orientation was associated with reduced anxiety and depression (chapter 6). The "OncoRev" single focused training had substantial and durable beneficial effects for quality of life, distress and one domain of problem-solving, i.e. negative problem orientation, but multi-modal rehabilitation did not show additional effects (chapters 4, 5, and 6).

\section{Discussion and conclusion}

In the general discussion, is concluded that the participants in the "OncoRev" program reported clinically relevant improvements in more domains of quality of life than the participants in the "Recovery \& Stability" program (chapters 2 and 5). Thus, in accordance with our expectations, the "OncoRev" self-management rehabilitation program seems more effective than the "Recovery and Stability" program. However, contrary to our expectations, multi-modal rehabilitation did not lead to benefits beyond those of single focused rehabilitation, neither in the short-term, nor in the long-term (chapters 4, 5, and 6).

The findings in this dissertation may be generalized to Dutch cancer survivors experiencing physical, psychological and/or social problems who usually apply for rehabilitation. The physical training comprised physical, as well as psychological and social elements because of the group-format and had a self-management approach. Therefore, this training probably functioned as an adequate biopsychosocial intervention. Due to the study designs in this dissertation, one may not conclude that cognitive-behavioral training by itself would not have been beneficial. Future research might compare cognitive-behavioral training versus non-intervention, self-management physical training versus traditional physical training, and individual selfmanagement physical training versus group-based self-management physical 
training. Furthermore, research might focus on costs and time effectiveness of rehabilitation and on enhancing accessibility of cancer rehabilitation.

To conclude, comprehensive group-based self-management physical training has substantial and durable positive effects on quality of life of cancer survivors who experience physical, psychological and/or social problems after their completion of primary medical treatment. Combining this selfmanagement physical training with cognitive-behavioral training did not show additional benefits, neither in the short-term nor in the long-term. Therefore, group-based self-management physical training is sufficient for most cancer survivors who apply for rehabilitation that includes physical training, and can be embedded in usual cancer care. 


\section{Samenvatting}

Doordat de incidentie van kanker toeneemt, en ook het percentage van de mensen die kanker overleven blijft groeien, moeten steeds meer mensen omgaan met kanker als met een chronische ziekte. Ongeveer $26 \%$ van de mensen die kanker overleven, rapporteert een verminderde kwaliteit van leven en geeft aan behoefte te hebben aan professionele hulp bij het omgaan met fysieke, psychische en sociale problemen. Verwacht wordt dat het aantal Nederlanders dat kanker overleeft en behoefte heeft aan revalidatie, zal stijgen van 4890 in het jaar 2000 naar minstens 6900 in het jaar 2015. Deze dissertatie beschrijft de ontwikkeling en evaluatie van revalidatieprogramma's voor overlevenden van kanker om hun kwaliteit van leven te verhogen. De belangrijkste doelen waren, ten eerste, de effecten te onderzoeken van multimodulaire fysieke en psychosociale revalidatie op de kwaliteit van leven van overlevenden van kanker en, ten tweede, te onderzoeken of multimodulaire fysieke en psychosociale revalidatie effectiever was dan enkel modulaire fysieke revalidatie. Eerst wordt de ontwikkeling en evaluatie beschreven van "Herstel \& Balans", een al langer bestaand multimodulair Nederlands oncologisch revalidatieprogramma (hoofdstukken 2 en 3). Daarna wordt de ontwikkeling en evaluatie van "OncoRev" gepresenteerd, een door de "OncoRev" projectgroep (pagina 4) recent ontwikkeld oncologisch revalidatieprogramma (hoofdstukken 4, 5, en 6). In deze hoofdstukken wordt multimodulaire revalidatie vergeleken met enkel modulaire revalidatie. De deelnemers aan beide revalidatieprogramma's waren overlevenden van kanker (alle kankersoorten) die hun primaire medische behandeling minstens 2 ("Herstel \& Balans") of 3 ("OncoRev") maanden geleden hadden afgesloten, een levensverwachting hadden van minstens 1 jaar, maar nog worstelden met fysieke, psychische en/of sociale problemen.

\section{De revalidatieprogramma's}

Het Nederlandse 12-weken durende groepsgewijze revalidatieprogramma "Herstel \& Balans" bestond uit fysieke trainingssessies (tweemaal per week), begeleid door fysiotherapeuten en 7 psychosociale sessies, waarin professionele begeleiders verschillende thema's behandelden. Omdat de 
ontwikkelaars van "Herstel \& Balans"de waarde erkenden van het biopsychosociale model en de theorie over sociale vergelijking, sociale steun en modeling, richtte dit multimodulaire programma zich op verbetering van de fysieke, psychologische en sociale domeinen van de kwaliteit van leven van overlevenden van kanker. Fysieke training was gericht op verbeteren van de mogelijkheden om te bewegen, toenemen van kracht en uithoudingsvermogen, omgaan met fysieke klachten zoals vermoeidheid en op bevorderen van gevoelens van controle en verminderen van stress. De fysieke "graduele activiteiten" training begon op een laag niveau en werd geleidelijk opgebouwd volgens een geïndividualiseerd trainingsschema. Iedere fysieke trainingssessie bestond uit individuele training van kracht en uithoudingsvermogen of een groepssport-en-spelprogramma, aqua aerobics in een binnenzwembad en een pauze waarin de deelnemers contact met elkaar konden hebben en ervaringen konden delen. De psychosociale sessies richtten zich op het bieden van steun bij het omgaan met kanker en het bevorderen van zelfvertrouwen en autonomie. De deelnemers kregen informatie over thema's die samenhingen met kanker en zij kregen de gelegenheid om hun ervaringen als overlevenden van kanker uit te wisselen. Het programma werd geïmplementeerd in 1996 in 2 revalidatiecentra. Op dit moment wordt oncologische revalidatie gebaseerd op dit multimodulaire programma, aangeboden in meer dan 60 centra in heel Nederland en België. Daarmee gaat oncologische revalidatie steeds meer deel uitmaken van de standaardzorg.

Gebaseerd op de "Herstel \& Balans" studies, ontwikkelde de "OncoRev" projectgroep (pagina 4) een nieuw programma. In 2004 startte in 4 Nederlandse centra het 12-weken durende groepsgewijze revalidatieprogramma "OncoRev". Zowel de fysieke trainingssessies (tweemaal per week) als de psychosociale sessies (eenmaal per week) van dit programma waren gebaseerd op zelfmanagement protocollen en werden begeleid door therapeuten. In dit programma werd de multimodulaire en de groepsgewijze aanpak behouden en daarnaast werden andere sociaal-cognitieve concepten geïntegreerd, zoals eigen-effectiviteit, zelfmanagement en theorie over oplossen van problemen. Het circulaire zelfmanagementproces omvat: 1) doelselectie, 2) informatieverzameling, 3) informatieverwerking en evaluatie hiervan, 4) beslissingen nemen, 5) actie en zelfreactie. De fysieke training bestond uit een persoonlijk trainingsprogramma gebaseerd op een maximale inspanningstest bij de start van het programma en op de persoonlijke doelen die de deelnemers stelden in samenwerking met hun therapeuten. Iedere sessie 
bestond uit individuele training gevolgd door groepssport. Tijdens de fysieke training gebruikten de deelnemers hartslagmeters, een schaal om kortademigheid en vermoeidheid te meten, trainingslogboeken om hun prestaties te monitoren en te evalueren. Ze ontvingen feedback, informatie en steun van hun therapeuten bij het reguleren van hun prestaties. Tijdens de psychosociale trainingssessies en in huiswerkopdrachten leerden de deelnemers hun zelfmanagementvaardigheden toe te passen bij het streven naar persoonlijke doelen (bijvoorbeeld in fysieke activiteiten zoals werk, huishouden, hobby's en in hun sociale contacten). De eerste 3 weken richtten de deelnemers zich vooral op het uitwisselen van ervaringen met kanker, op psycho-educatie over stress, ontspanning, vermoeidheid, trainingsfysiologie en ziekte percepties en op het bevorderen van optimisme en eigen-effectiviteit ten aanzien van zelfmanagement. Daarna werden de deelnemers vooral getraind in het oefenen van de stappen in het circulaire proces van probleemoplossing: 1) probleemoriëntatie, 2) probleemdefinitie en probleemformulering, en doelen stellen, 3) bedenken van mogelijke oplossingen (brainstormen), 4) beslissingen nemen, 5) uitvoeren van oplossingen en evaluatie hiervan.

\section{De studies}

Een longitudinale cohortstudie (1996-2002; $\mathrm{n}=658)$ onderzocht de effecten van het "Herstel \& Balans" programma op de kwaliteit van leven van overlevenden van kanker (hoofdstuk 2). De meeste deelnemers (78\%) waren vrouwen en bij de helft van de deelnemers was borstkanker gediagnosticeerd. De gemiddelde leeftijd was 51 jaar en de deelnemers begonnen met de revalidatie gemiddeld 1.3 jaar nadat zij de primaire medische behandeling hadden afgesloten. De geringe uitval van deelnemers (4\% halverwege en $13 \%$ na 12 weken) geeft aan dat het programma haalbaar was en dat de deelnemers een hoge motivatie hadden. Halverwege de revalidatie werden er significante verbeteringen gevonden in alle domeinen van kwaliteit van leven, behalve in cognitief functioneren. $\mathrm{Na}$ de revalidatie rapporteerden de deelnemers significante verbeteringen in alle domeinen van kwaliteit van leven. Deze verbeteringen waren echter slechts klinisch relevant voor 2 subgroepen van deelnemers, namelijk voor niet werkende deelnemers in rol functioneren en voor deelnemers met andere kankerdiagnoses dan borstkanker in fysiek functioneren en sociaal functioneren. De "Herstel \& Balans" studie betrof een grote onderzoekspopulatie en liet positieve effecten van revalidatie zien in gemengde groepen van overlevenden van kanker (alle 
kankersoorten). Vervolgens werd in een focusgroepstudie geëxploreerd wat de zienswijze was van vroegere deelnemers aan "Herstel \& Balans" op hoe en in welke mate dit programma had voldaan aan hun behoeften (hoofdstuk 3). Deze studie beschreef hun ervaringen gedurende en na het programma, hun visie op de waarde van oncologische revalidatie en hun suggesties voor verbetering van het programma. De focusgroepdeelnemers representeerden de kenmerken van de longitudinale studiepopulatie bij aanvang van het programma. Zij waardeerden de revalidatie als een belangrijke springplank in hun voortgaande herstelproces. Daarnaast zagen zij de combinatie van fysieke en psychosociale sessies en de groepsgewijze aanpak als sterke punten van het "Herstel \& Balans" programma en zij suggereerden dat het programma zich meer zou moeten richten op het ontwikkelen van vaardigheden om met kanker om te gaan. De studies in hoofdstuk 2 en 3 suggereerden dat multimodulaire revalidatie haalbaar en effectief was in gemengde groepen van overlevenden van kanker, maar dat er ruimte was voor verbetering van het programma. Daarnaast vond de "OncoRev" projectgroep het noodzakelijk de verschillende modules te evalueren en de revalidatiegroepen te vergelijken met een groep die geen revalidatie volgde. Onze hypotheses waren dat het integreren van cognitief-gedragsmatige elementen in fysieke training de effecten van fysieke training op kwaliteit van leven zou verbeteren, en dat het combineren van zo'n uitgebreide fysieke training met cognitief-gedragsmatige psychosociale training zou leiden tot nog grotere verbeteringen in de kwaliteit van leven van overlevenden van kanker.

In de gerandomiseerde gecontroleerde "OncoRev"studie (2004-2007) werd bij overlevenden van kanker onderzocht wat de effecten waren van enkel modulaire revalidatie $(n=71)$ en van multimodulaire revalidatie $(n=76)$ op kwaliteit van leven, oplossen van problemen en op angst en depressie, direct na de revalidatie en 3 maanden en 9 maanden na revalidatie (hoofdstuk 4, 5, en 6) Enkel modulaire revalidatie bestond uit uitgebreide op zelfmanagementprincipes gebaseerde fysieke training en multimodulaire revalidatie omvatte zowel uitgebreide op zelfmanagementprincipes gebaseerde fysieke training als op zelfmanagementprincipes gebaseerde cognitief-gedragsmatige training. Daarnaast werden direct na de revalidatie de effecten van beide revalidatiegroepen vergeleken met een wachtlijst-vergelijkingsgroep $(n=61)$ (hoofdstuk 4).

De meeste deelnemers (86\%) waren vrouwen en bij de helft van de deelnemers (57\%) was borstkanker gediagnosticeerd (hoofdstuk 4). De gemiddelde leeftijd was 50 jaar en de deelnemers begonnen met de revalidatie gemiddeld 
1.5 jaar na de primaire medische behandeling. De uitval van deelnemers was laag: $8 \%$ van de deelnemers in de revalidatiegroepen direct na revalidatie, $10 \% 3$ maanden na revalidatie, en 13\% 9 maanden na revalidatie (hoofdstuk 5). Direct na de revalidatie rapporteerden de deelnemers in de revalidatiegroepen, in vergelijking met de wachtlijst-vergelijkingsgroep, significante en klinisch relevante verbeteringen in vooral de fysieke domeinen van kwaliteit van leven, namelijk een vermindering in rolbeperkingen door fysieke problemen, beter fysiek functioneren, toegenomen vitaliteit en verbeterde gezondheid (hoofdstuk 4). Op geen enkel meetmoment waren er significante verschillen tussen de effecten van multimodulaire en van enkel modulaire revalidatie op de kwaliteit van leven van de deelnemers (hoofdstuk 4 en 5). Vergeleken met de situatie bij de start van revalidatie, rapporteerden de deelnemers in de revalidatiegroepen op alle meetmomenten significante verbeteringen in alle domeinen van leven (hoofdstukken 4 en 5). Deze verbeteringen waren ook 9 maanden na de revalidatie nog klinisch relevant in vrijwel alle domeinen van kwaliteit van leven (hoofdstuk 5).

Vergeleken met de situatie bij de start van revalidatie, rapporteerden de deelnemers in de revalidatiegroepen daarnaast op alle meetmomenten een significante verbetering in één domein van oplossen van problemen, namelijk minder negatieve probleemoriëntatie, en significant minder angst en depressie (hoofdstuk 6). Daarnaast bleek dat de multimodulaire revalidatie ook op het oplossen van problemen en op angst en depressie niet significant meer positieve effecten had dan de enkel modulaire revalidatie. Daaropvolgend werden de effecten van de interventies op deelnemers met hogere niveaus van angst en depressie vergeleken met deelnemers met lagere niveaus van angst en depressie bij aanvang van revalidatie. Deze analyses lieten op geen enkel meetmoment significante verschillen zien in het oplossen van problemen en het niveau van angst en depressie (hoofdstuk 6). Verminderde negatieve probleemoriëntatie was geassocieerd met verminderde angst en depressie (hoofdstuk 6). De enkel modulaire "OncoRev" training had substantiële en aanhoudende positieve effecten op kwaliteit van leven, angst, depressie en op één domein van oplossen van problemen, namelijk negatieve probleemoriëntatie, maar multimodulaire revalidatie liet geen extra effecten zien (hoofdstuk 4, 5 en 6). 


\section{Discussie en conclusie}

In de algemene discussie van deze dissertatie wordt geconcludeerd dat de deelnemers aan het "OncoRev" programma in meer domeinen van kwaliteit van leven klinisch relevante verbeteringen rapporteerden dan de deelnemers aan het "Herstel \& Balans" programma (hoofdstukken 2 en 5). In overeenstemming met onze verwachtingen lijkt het op zelfmanagementprincipes gebaseerde "OncoRev" revalidatieprogramma dus effectiever dan het "Herstel \& Balans" programma. Echter, in tegenstelling tot onze verwachtingen leidde het multimodulaire "OncoRev" revalidatieprogramma niet tot betere resultaten dan het enkel modulaire "OncoRev" revalidatieprogramma, noch op de korte noch op de lange termijn (hoofdstukken 4, 5, en 6). De bevindingen in deze dissertatie kunnen worden gegeneraliseerd naar de Nederlandse overlevenden van kanker die fysieke, psychische en/of sociale problemen ervaren en die zichzelf doorgaans aanmelden voor revalidatie. De fysieke training omvatte zowel fysieke, psychologische en sociale elementen door de toepassing van zelfmanagementprincipes en een groepsgewijze aanpak. Waarschijnlijk functioneerde deze training daardoor al als een adequate bio-psychosociale interventie. Door de opzet van de studies in deze dissertatie kan men niet concluderen dat cognitief-gedragsmatige training op zichzelf geen positieve resultaten heeft. Toekomstig onderzoek zou zich kunnen richten op vergelijkingen van cognitief-gedragsmatige training versus geen interventie, op fysieke training gebaseerd op zelfmanagementprincipes versus traditionele fysieke training, en op individuele op zelfmanagementprincipes gebaseerde fysieke training versus groepsgewijze op zelfmanagementprincipes gebaseerde fysieke training. Daarnaast zou toekomstig onderzoek zich kunnen richten op evaluatie van de effectiviteit van revalidatie qua kosten en tijd, en op het bevorderen van de toegankelijkheid van oncologische revalidatie.

Geconcludeerd kan worden dat groepsgewijze op zelfmanagementpricipes gebaseerde fysieke training substantiële en aanhoudende positieve effecten heeft op de kwaliteit van leven van overlevenden van kanker die fysieke, psychische en/of sociale problemen ervaren na de primaire medische behandeling. De combinatie van deze op zelfmanagementprincipes gebaseerde fysieke training met cognitief-gedragsmatige training liet geen toegevoegde effecten zien, noch op de korte noch op de lange termijn. Groepsgewijze op zelfmanagementprincipes gebaseerde fysieke training is voldoende voor de meeste overlevenden van kanker die zichzelf aanmelden voor revalidatie die fysieke training omvat en kan worden opgenomen in de standaard oncologische zorg. 


\section{Dankwoord}

Mijn dank gaat in de eerste plaats uit naar alle mensen die deelnamen aan de revalidatieprogramma's die in dit proefschrift geëvalueerd werden. U werkte aan uw eigen herstel en bevorderde tegelijkertijd de mogelijkheden tot herstel van anderen die met kanker geconfronteerd worden. Dank voor uw medewerking bij groepsinterviews, fysieke testen, en vragenlijstmetingen vóór, tijdens en vaak nog lang na de revalidatie, in tijden van voorspoed, maar ook als het niet goed met $\mathrm{u}$ ging. De persoonlijke contacten met $\mathrm{u}$ boden mij extra inzichten, en motiveerden mij steeds weer, omdat $u$ mij hielp te blijven beseffen waarom en voor wie we deze studies deden.

De leden van de Beoordelingscommissie, voorzitter prof. dr. N.K de Vries, prof. dr. H. Hillen, prof. dr. M. von Meyenfeldt, prof. dr. J.F.A. Pruyn en prof. R.M. Ryckman dank ik voor de beoordeling van het proefschrift. Heel graag bedank ik mijn promotor prof.dr H.W. van den Borne en copromotor dr. I Mesters voor de uitstekende begeleiding gedurende het hele promotietraject. Bart en Ilse, voor mij vormden jullie een goed team, waarin jullie elkaar in veel opzichten aanvulden. Ik waardeer het zeer dat ik veel vrijheid kreeg en tegelijk steeds, door dik en dun, kon rekenen op persoonlijke aandacht, bemoediging, nieuwe ideeën en een kritische blik van ieder van jullie. En gelukkig werd er ook gelachen, als ik éven binnenviel bij Ilse, of met Bart Hoog Catharijne doorkruiste in gezwinde pas! Ik vond het destijds fantastisch dat ik jullie voorstel voor onderzoek naar oncologische revalidatie uit kon gaan voeren. En nog steeds is het geweldig om te bevorderen dat mensen zelf de regie weer veroveren als ze geconfronteerd zijn met ingrijpende gebeurtenissen in hun leven. Indertijd dachten we in Lim- 
burg onderzoek te doen, gebaseerd op "Herstel \& Balans", maar al snel breidden we uit.

Aan de wieg van "Herstel \& Balans" stond het Integraal Kankercentrum Limburg, en in het bijzonder drs. B.C.M. Gijsen. Brigitte, ik waardeer het zeer dat jij indertijd bij dit bijzondere initiatief ook direct dacht aan de voorwaarden voor een wetenschappelijke evaluatie, en later, in het kader van de Stichting "Herstel \& Balans" consequent bent blijven werken aan de voortgaande ontwikkeling, implementatie, kwaliteitsborging en financiering van oncologische revalidatie in Nederland. Je bijdrage als co-auteur bij twee hoofdstukken in dit proefschrift illustreren dit. De financiering door de Universiteit Maastricht en het Integraal Kankercentrum Limburg maakte de "Herstel \& Balans" studies mogelijk.

"Herstel \& Balans" werd in 1996 opgezet door het Integraal Kankercentrum Limburg in nauwe samenwerking met het Sport Gezondheidscentrum van de Stichting Revalidatie Limburg in Hoensbroek, en in 1998 startte ook Astmacentrum Hornerheide in Horn met het programma. $\mathrm{Nu}$ kunnen mensen al deelnemen in 60 plaatsen in Nederland en België. Veel medewerkers in Hoensbroek en Hornerheide zetten zich in voor de revalidatie en het onderzoek. Een speciale vermelding verdient de heer Peter Heemskerk die als directeur van het Sport Gezondheidscentrum met groot enthousiasme zorgde voor het eerste protocol en voor de voortgaande ontwikkeling van het programma. Peter, ik weet hoe je samen met je medewerkers veel moeite hebt gedaan om ook aan de "OncoRev" studie deel te nemen. Tevergeefs, "Herstel \& Balans" was in Limburg al zo ingeburgerd dat we hier onvoldoende mensen at random konden indelen in de verschillende "OncoRev" onderzoeksgroepen.

De "OncoRev" projectgroep, bestaand uit onderzoekers van de Universiteit Maastricht, het Universitair Medisch Centrum Utrecht, het Erasmus Medisch Centrum Rotterdam, het Universitair Medisch Centrum Groningen, het Integraal Kankercentrum Noord-Nederland en de Hanze Hogeschool Groningen, kon een nationale oncologische revalidatiestudie ontwikkelen, voortbouwend op de ervaringen in Zuid en Noord-Nederland. Voor haar bijdrage aan de "OncoRev" project aanvraag dank ik drs. M.E. Remie van het Integraal Kankercentrum Rotterdam, en voor hun bijdrage aan de voorbereiding van het onderzoek dank ik de Vereniging van Integrale Kanker Centra, de coördinatoren revalidatie en nazorg van alle Integrale Kankercentra, de Stichting Herstel \& Balans, en de vele experts in klankbord groepen. De financiering door de Universiteit Maastricht, KWF Kankerbe- 
strijding, en het Integraal Kankercentrum Noord-Nederland maakte "OncoRev" mogelijk.

In 4 instellingen hielpen diverse medewerkers ons bij de ingewikkelde "OncoRev" logistiek, en aanwezige therapeuten schoolden zich in de nieuwe fysieke en cognitief-gedragsmatige trainingen, verzorgden de trainingen, en gaven ons gedegen feedback op de protocollen. Ik dank al deze enthousiaste mensen in Sport en Fitnesscentrum De Hoogstraat en het Helen Dowling Instituut in Utrecht, in Ziekenhuis Hilversum, locatie Zonnestraal, in Universitair Medisch Centrum Groningen, locatie Beatrixoord, en in Erasmus Medisch Centrum Rotterdam (afdeling Revalidatie, Dienst Psychosociale Zorg, Afdeling Medische Psychologie en Psychotherapie, Afdeling fysiotherapie, afdeling Hematologie, Unit 7 Noord, Unit 5 Zuid, en afdeling Chirurgie Daniel den Hoed). Ik bewaar goede herinneringen aan de scholingsdagen en de talrijke telefoongesprekken en bezoeken aan jullie, vóór, tijdens en na het programma. Dank aan prof. dr. F. Backs voor de mogelijkheid om inspanningstesten voor de locatie Zonnestraal te verrichten bij Revalidatie en Sportgeneeskunde, Universitair Medisch Centrum Utrecht. Dank aan de instellingen die ons in staat stelden in korte tijd de deelnemers voor de wachtlijstcontrolegroep te werven: Astmacentrum Ermelo, Blixembosch Revalidatiecentrum Eindhoven, Elkerliek Ziekenhuis, Fysiotherapie Oude Gracht, Jeroen Bosch Ziekenhuis, locatie GZG \& WA, Revalidatiecentrum Leijpark, Sportcentrum Groot Klimmendaal, Sportinstituut Frits v.d. Werff, Twenteborgziekenhuis, en Ziekenhuis Bernhoven, locatie Oss.

Dr. W.J.G. Ros, beste Wynand, ik dank jou als projectleider van "OncoRev" voor je goede zorgen voor het welslagen van dit project, waar zoveel mensen zich sterk betrokken bij voelden, zowel binnen als buiten de projectgroep. Dank aan mijn mede onderzoekers, dr. A.M. May en dr. E. van Weert. Anne, je nam op geheel eigen wijze iedereen voor je in, en organiseerde soepel "OncoRev". Leuk, die meetdagen in Rotterdam en Utrecht: we genoten allebei van het contact met de deelnemers. Ellen, ik denk nog met veel plezier terug aan de avontuurlijke periode waarin we self-management in "OncoRev" vorm gaven. Over self-management zijn wij en de revalidatiewereld nog niet uitgesproken. Dr. J.E.H.M. Hoekstra-Weebers en dr. C.P. van der Schans, copromotoren van Ellen, beste Josette en Cees, veel dank voor jullie bijdragen aan het hele project. We werden allemaal getroffen door het volkomen onverwachte overlijden van prof.dr. R. W. Trijsburg tijdens zijn vakantie in Schotland op 8 april 2007. Zijn taak als promotor van Anne werd overgenomen door prof. dr J. Passchier en prof.dr D.E. Grobbee. 
Dank aan alle projectgroepleden als co-auteurs van de hoofdstukken over "OncoRev".

Dr. J.H.C. van den Hout, Anja, jij bracht mij al snel in contact met problemsolving. We werkten aan de vertaling en inpassing van jouw protocol in de cognitief-gedragsmatige interventie voor "OncoRev". Jij bracht me in contact met andere onderzoekers en therapeuten die al met problem-solving werkten, we verzorgden de scholingsdagen in Utrecht en je bent co-auteur van hoofdstuk 6. Kortom, een vruchtbaar, leuk, en gezellig contact dat we vast behouden. Dr. R.J.E.M. Smeets in Blixembosch Revalidatiecentrum Eindhoven en dr. R. Severeijns in Academisch Ziekenhuis Maastricht, beste Rob en Rudy, dank voor de ruime mogelijkheden om de toepassing van problem-solving training bij jullie patiënten te kunnen observeren. Prof.dr. G.I.J.M. Kempen en prof.dr. J.T van Eijk, Universiteit Maastricht, beste Ruud en Jacques, hartelijk dank voor jullie adviezen over kwaliteit van leven, de HADS en self-management. Dank aan de onafhankelijk artsen dr. P. Portegijs, Universiteit Maastricht, bij de kwalitatieve studie en dr. A. de Graeff, Universitair Medisch Centrum Utrecht bij "OncoRev". Piet, fijn dat je alweer onafhankelijk arts wilde zijn, en dank voor zoveel meer.

Een speciaal woord van dank voor dr. F. Tan, Universiteit Maastricht als statisticus en co-auteur van hoofdstuk 4. Frans, ik kon eigenlijk altijd bij je terecht en, je wist steeds zo goed aan te sluiten bij mijn vragen, dat je me steeds beter in staat stelde zelf ingewikkelde statistische problemen te analyseren en op te lossen. Het was feestelijk om zo vrolijk zoveel van je te kunnen leren. Naast jou bedank ik ook alle andere docenten voor hun waardevolle promovendi cursussen.

Lara Heuveling van de afdeling Datamanagement, Universitair Medisch Centrum Utrecht, en Anita Legtenberg van MEMIC Maastricht dank ik heel hartelijk voor het zorgvuldige datamanagement. Anita, je wist nauwgezetheid, betrouwbaarheid, en flexibiliteit goed te combineren en ik was blij met de prettige wijze waarop je mij vele handige tips gaf.

In de afgelopen jaren zijn een aantal gezondheidswetenschappers afgestudeerd in GVO, nadat ze als stagiaires hadden meegewerkt aan de studies in dit proefschrift: Evelien van der Peet, Eveline van Utteren, Paulien van Duren, Tessa van den Akker en Diny Winkels. Ik vond het erg leuk om met jullie allemaal samen te werken en te leren en jullie te kunnen toespreken bij jullie bul uitreiking. Dank ook aan de stagiaires van Anne en Ellen voor hun bijdragen aan "OncoRev".

Goede "ondersteuners" op administratief, financieel, en ICT gebied zijn onontbeerlijk bij ieder onderzoek. Daarom mijn hartelijke dank aan Marja 
Vissers, Helga Happaerts, Josée Trottemant, Anton Janssen, en Leon Kolenburg. Marja, extra dank voor je voorbereidingen van de promotie. Leon, ik zet jou graag extra in het zonnetje. Voor veel GVO-ers ben je een geweldige steun en toeverlaat, zo constateerde Ella al toen ze in 2004 tegenover jouw kamer de "OncoRev" werkboeken samen stelde. Voor mij was je onmisbaar met je hulp bij computer perikelen, verzenden van vragenlijsten, vormgeven van flowcharts en andere lastige figuren, en audiovisuele apparatuur kwesties, afgewisseld met veel levenswijsheid, grapjes en de voetbaltoto. Tussendoor zette jij ook nog de "OncoRev" website in elkaar. Iedere vakgroep krijgt vleugels met iemand als jij op de werkvloer.

Mijn GVO collega's, dank voor het aangenaam vertoeven. Speciaal dank aan mijn kamergenote Resie Knops voor het delen van groot en klein lief en leed. Ik verheug me op jouw feestje! Bilbo Schickenberg, 's ochtends welkom en 's avonds afscheid: zo blijf je terugkomen, dank je wel voor je foto's en "IreneforOscar" montages. Cristina Quevedo, we keep in touch. Janneke Harting en Daksha van Dijck, moedige meiden, natuurlijk doen we dat.

In 2007 combineerde ik mijn promotietraject met kwalitatief onderzoek naar de kwaliteit van zorg (CQI), met mede-onderzoeker Albine Moser, bij dr. T. van der Weijden en dr. H. Tange, Vakgroep Huisartsgeneeskunde, Universiteit Maastricht. Trudy, en Huibert en Albine, we zeiden het al vaker, dit flitsende resultaatrijke project was een waar feest, waarin we veel van elkaar leerden en een geweldig leuke tijd hadden. Dank ook aan de deelnemers, en aan Bernadette Zinzen, Karin Vaessen en Paddy Hinssen, en aan Elly Stoffers voor de wonderbaarlijke werving. Albine, geweldig dat we nu weer collega's zijn: we vervolgen onze quarrels, sorry, "scientific debates".

Ik prijs me gelukkig opnieuw onderwijs en onderzoek te kunnen combineren op de Academie Verloskunde Maastricht met een team van inspirerende en bijzondere collega's. Dagelijks voel ik in de contacten op de Academie dat de verloskunde één van de meest veelbelovende en boeiende werkvelden is. Fijn zo hartelijk opgenomen te zijn in de Department Midwifery Science onder leiding van Marianne Nieuwenhuijze. Geweldig om onderwijs en onderzoeksplannen te smeden met vroegere GVO-collega's, Marlein Ausems en Evelien van Limbeek, en met nieuwe collega's.

Anita Botterweck en Yolanda Maat, mijn paranimfen, collega's in gezondheidwetenschappen en moederschap, hardloopmaatjes en vooral vriendinnen. Fijn dat we al zo lang samen oplopen in "The Dutch Mountains" en door weer en wind vrolijk doorgaan ... of worden. 
Lieve vrienden, en vrienden die bijna familie zijn, Babs, Hubert en Nel, vaak ben ik blij dat we al zo veel van onze levens konden delen, en, zo staat nu zwart op wit, we blijven prachtige geschiedenis schrijven. Familie, in het bijzonder Mam, Pap, Anneke, Jacqueline, Bert, Tom, Riet, Mark, Mireille, Elly, Harry, Els, Fieke, Michel, Manuel, Karin, en Paul, dank voor het vormen van een goed thuis, samen met al jullie kinderen.

Steven, Ella en Sacha, geen groter geluk dan jullie te mogen zien opgroeien, mij voorbij te zien komen of, nog leuker, heel andere wegen te zien gaan..., maar dat weten jullie allang. Rianne en Steven, ik zie jullie graag zo sprankelen.

Wat een weelde om ons geluk te delen, Jelle. 


\section{Curriculum Vitae}

Irene Korstjens was born August $14^{\text {th }} 1955$ in The Hague, the Netherlands. After secondary school (HAVO) at the Huygens Lyceum in Voorburg, she studied Social Work at the Social Academy in The Hague (1977). The next ten years she worked as a social worker in primary care in Rijswijk and in multidisciplinary secondary care for physically and mentally retarded children in Rotterdam. She obtained a post-doctoral certificate Advanced Social Work System Therapy (IVABO, 1983) in Amsterdam and a postdoctoral certificate Advanced Social Work Mental Health (VSID-MW, 1986) in Leusden. In 1987, she moved with Jelle Stoffers to Maastricht, where in the next five years their three children were born. In the South of the Netherlands, between 1987 and 1996, she was a supervisor for students Advanced Social Work Mental Health, and a social worker in primary care (Sittard), in secondary care for mentally retarded children (Sittard), and at a school for children with hearing and language difficulties (Hoensbroek). In those years, she also took courses in statistics and psychology at the Open Universiteit Nederland. She then studied Health Sciences at Maastricht University and obtained her Master's degree in Mental Health Sciences (2001). In November 2001, she started the PhD study presented in this dissertation at the Department of Health Education and Promotion at Maastricht University. During her Master study and her $\mathrm{PhD}$ study she was engaged in teaching at the Faculty of Health, Medicine, and Life Sciences, Maastricht University. In 2007 she worked as a researcher at the Department of General Practice, Maastricht University. Since December 2007 she is working as lecturer and researcher at the Department of Midwifery Science, University of Midwifery Education \& Studies Maastricht. 



\section{Publications}

Korstjens, I., May, A. M., Van Weert, E., Mesters, I., Tan, F., Ros, W. J. G., HoekstraWeebers, J. E. H. M., Van der Schans, C.P., \& Van den Borne, B. (2008). Quality of life after self-management cancer rehabilitation: a randomized controlled trial comparing physical and cognitive-behavioral training versus physical training. Psychosomatic Medicine, 70, 422-9. DOI:10.1097/PSY.0b013e31816e038f.

Korstjens, I., Mesters, I., Gijsen, B. C. M., \& Van den Borne, B. (2008). Cancer patients' view on rehabilitation and quality of life: a programme audit. European Journal of Cancer Care, 7, 290-7. DOI: 10.1111/j.1365-2354.2007.00864.x.

Korstjens I., Mesters I. van der Peet E., Gijsen B., \& Van den Borne B. (2006). Quality of life of cancer survivors after physical and psychosocial rehabilitation. European Journal of Cancer Prevention, 15, 541-7.

May, A. M., Duivenvoorden, H. J., Korstjens, I., Van Weert, E., Hoekstra-Weebers, J. E. H. M., Van den Borne, B., Mesters, I., Van der Schans, C.P. \& Ros, W.J.G. (2007). The effect of group cohesion on rehabilitation outcome in cancer survivors. PsychoOncology, DOI: 10.1002/pon.1308.

May, A. M., Van Weert, E., Korstjens, I., Hoekstra-Weebers, J. E. H. M., Van der Schans, C. P., Zonderland, M. L., Mesters, I., Van den Borne, B., \& Ros, W. J. G. (2008). Improved physical fitness of cancer survivors: a randomized controlled trial comparing physical training with physical and cognitive-behavioural training. Acta Oncologica, 47, 825-34. DOI: 10.1080/02841860701666063.

Van Weert, E., Hoekstra Weebers, J. E. H. M., May, A. M., Korstjens, I., Ros, W. J. G., \& Van der Schans, C. P. (2008). The development of an evidence-based physical selfmanagement rehabilitation programme for cancer survivors. Patient Education and Counseling, 71, 169-90. DOI: 10.1016/j.pec.2007.11.027.

May, A. M., Korstjens, I., Van Weert, E., Van den Borne, B., Hoekstra-Weebers, J. E. H. M., Van der Schans, C. P., Mesters, I., Passchier, J., Grobbee, D.E., \& Ros, W. J. G. Longterm effects on cancer survivors' quality of life of physical training versus physical training combined with cognitive-behavioral therapy: results from a randomized trial. Submitted.

May, A. M., Van Weert, E., I.Korstjens, I., Hoekstra-Weebers, J. E. H. M., Van der Schans, C.P., Zonderland M.L, C. P., Mesters, I., Van den Borne, B., \& Ros, W. J. G. Maximal and submaximal exercise outcome following physical training in cancer survivors: a submaximal exercise test as an alternative for an exhaustive exercise test? Submitted.

Korstjens, I., Mesters, I., May, A. M., Van Weert, E., Van den Hout, J.H.C., Ros, W. J. G., Hoekstra-Weebers, J. E. H. M., Van der Schans, C.P., \& Van den Borne, B. Effects of cancer rehabilitation on problem-solving and distress: a RCT comparing physical and cognitive-behavioral training versus physical training. To be submitted as a shorter version of chapter 6 . 